\title{
Lower bounds for Auslander's representation dimension
}

\author{
INAUGURAL - Dissertation
}

\author{
Zur \\ Erlangung des Doktorgrades \\ der Mathematisch-Naturwissenschaftlichen Fakultät \\ der Universität zu Köln
}

vorgelegt von

Steffen Oppermann

aus San Jose (Kalifornien) 
Berichterstatter: Prof. Dr. Steffen König

Prof. Dr. Peter Littelmann

Prof. Dr. Idun Reiten

Tag der mündlichen Prüfung: 25.10.2007 


\section{Kurzzusammenfassung}

Der Begriff der Darstellungdimension einer endlichdimensionalen Algebra wurde von Auslander eingeführt. Er zeigte, dass eine Algebra genau dann endlichen Darstellungstyp hat, wenn ihre Darstellungsdimension höchstens zwei ist, und erwartete, dass die Darstellungdimension darstellungsunendlicher Algebren ein Maß dafür sein sollte, wie weit die betreffende Algebra davon entfernt ist, darstellungsendlich zu sein.

Währen in vielen Fällen, und dank Iyama auch im Allgemeinen, obere Schranken für die Darstellungsdimension bekannt sind, gibt es nicht-triviale untere Schranken bisher nur für zwei (ähnlich konstruierte) Serien von Algebren. In dieser Arbeit werden neue untere Schranken für Auslanders Darstellungsdimension konstruiert und allgemeinere Methoden hierfür entwickelt.

Zunächst betrachten wir die Gruppenalgebren elementarabelscher Gruppen und zeigen, dass ihre Darstellungsdimension größer als der Rang der Gruppe ist. Daraus ergibt sich ein Beweis einer Vermutung von Benson bezüglich der Loewylänge von Blöcken von Gruppenalgebren.

Dann wird ein Dimensionsbegriff für Modulkategorien eingeführt. Dieser ermöglicht es, bessere untere Schranken für die Darstellungsdimension zu finden, und insbesondere erstmals für nicht selbstinjektive Algebren mit Darstellungsdimension größer als drei den genauen Wert der Darstellungsdimension zu bestimmen.

Schließlich geben wir ein allgemeines Kriterium, um untere Schranken für Auslanders Darstellungsdimension zu finden: Wenn es eine Familie von Moduln gibt, so dass genügend viele Mitglieder dieser Familie bestimmte $d$ Erweiterungen haben, dann ist die Darstellungsdimension mindestens $d+2$. Es wird an einigen interessanten Familien von Algebren gezeigt, wie das Kriterium angewendet wird, und dass es häufig den richtigen Wert für die Darstellungsdimension liefert. 


\section{Abstract}

The notion of representation dimension of a finite dimensional algebra has been introduced by Auslander. He has shown that an algebra is of finite representation type if and only if its representation dimension is at most two. He has expected that the representation dimension of a representation infinite algebra should measure how far this algebra is from being representation finite.

In many cases, and, thanks to Iyama, also in general, upper bounds for the representation dimension are known. However there are non-trivial lower bounds only for two (similarly constructed) series of algebras. In this thesis new lower bounds for Auslander's representation dimension are constructed, and more general methods are developed.

First we consider group algebras of elementary abelian groups. We show that their representation dimension is greater than the rank of the group. This leads to a proof of a conjecture of Benson concerning the Loewy length of blocks of group algebras.

Then a notion of dimension of a module category is introduced. This notion enables us to find better lower bounds for the representation dimension. In particular it allows us for the first time to determine the value of the representation dimension of non-self-injective algebras of representation dimension greater than three.

Finally we give a general criterion for finding lower bounds for Auslander's representation dimension: Suppose there is a family of modules, such that sufficiently many members of the family have certain $d$-extensions. Then the representation dimension of the algebra is at least $d+2$. We illustrate how this criterion is applied on some interesting families of algebras. It turns

out that the lower bound is often the precise value of the representation dimension. 


\section{Contents}

1 Introduction 4

2 Background 11

2.1 Auslander's representation dimension . . . . . . . . . . . . . . 11

2.2 The dimension of a triangulated category . . . . . . . . . . . . 14

2.3 Geometry . . . . . . . . . . . . . . . . . . 23

2.4 Known examples of algebras with representation dimension larger than three . . . . . . . . . . . . . . 23

3 Group algebras $\quad 25$

3.1 Elementary abelian groups . . . . . . . . . . . . 25

3.2 Finite groups in general — Benson's Conjecture . . . . . . . . 31

4 The dimension of a module category 33

5 A criterion for finding lower bounds for the dimension of $\begin{array}{ll}\text { module categories } & 40\end{array}$

5.1 The general criterion . . . . . . . . . . . . . . . . . 40

5.2 A practical version of the criterion . . . . . . . . . . . . 45

5.3 Coverings ...................... . . 49

6 Iyama's upper bound for the representation dimension 53

7 New and improved examples of algebras of representation $\begin{array}{ll}\text { dimension at least four } & 57\end{array}$

7.1 Examples related to exterior algebras . . . . . . . . . . . 57

7.2 Examples related to truncated polynomial rings . . . . . . . . 62

7.3 Further examples . . . . . . . . . . . . . . . 66

$\begin{array}{lll}8 & \text { Open questions } & \mathbf{7 0}\end{array}$ 


\section{Introduction}

In 1971, Auslander [2] has introduced the notion of representation dimension of a finite dimensional algebra. His definition is as follows:

Definition. The representation dimension of a finite dimensional algebra $\Lambda$ is

$\operatorname{repdim} \Lambda=\min \left\{\operatorname{gld} \operatorname{End}_{\Lambda}(M) \mid M\right.$ is a finite dimensional $\Lambda$-module and generates and cogenerates the category of finite dimensional $\Lambda$-modules

Here $\operatorname{End}_{\Lambda}(M)$ is the $\Lambda$-endomorphism ring of $M$ and gld denotes the global dimension of a ring, that is the supremum of the projective dimensions of all modules over that ring.

Auslander has shown that an algebra is of finite representation type, that is, it admits only finitely many indecomposable modules up to isomorphism, if and only if its representation dimension is at most 2. We will give a proof of this fact as Corollary 2.1.3. This led Auslander to the expectation, that the representation dimension of a representation infinite algebra should measure how far this algebra is from having finite representation type.

Unfortunately, it turned out to be rather hard to compute the actual value of the representation dimension of a given algebra. However, in 2003 Iyama [11] has given a proof that the representation dimension of a finite dimensional algebra is always finite. He did so by explicitly constructing a module $M$ with gld $\operatorname{End}_{\Lambda}(M)$ finite, so the minimum in the definition above is always finite. When applied to a given algebra, this method yields an upper bound for the representation dimension of this algebra. Here we will explain Iyama's method in Section 6 and in particular as Theorem 6.1.

By Auslander's result mentioned above, it was known that any representation infinite algebra has representation dimension at least three. However, it was not known whether numbers greater than three can occur as the representation dimension of a finite dimensional algebra, until Rouquier [16] has shown in 2005 that the representation dimension of the exterior algebra of an $n$-dimensional vector space is always $n+1$. This fact will be explained here as Theorem 2.4.1. His result shows that any natural number $(\neq 1)$ occurs as the representation dimension of some algebra. In order to show that the representation dimension of the exterior algebra is at least $n+1$, Rouquier introduced the notion of dimension of a triangulated category, and showed that the dimension of the bounded derived category, and, for self-injective algebras, of the stable module category, can be used to establish lower bounds 
for the representation dimension. This method will be explained in detail in 2.2 .

Using similar methods, Krause and Kussin [12] have been able to establish a lower bound for another family of algebras. They have shown that the representation dimension of the algebra $k Q / I$, with

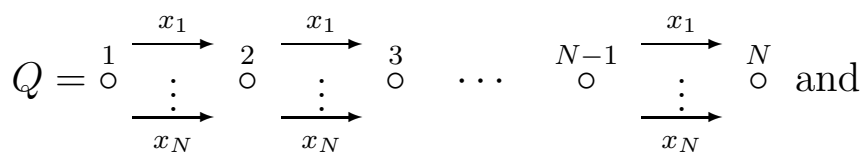

$$
\begin{aligned}
& I=\left(x_{n} x_{m}-x_{m} x_{n}\right) \text {. }
\end{aligned}
$$

has representation dimension at least $N-1$. The case $N=2$ indicates that this lower bound is not the precise value.

The main aim of this thesis is to improve and generalize the methods of the two papers mentioned above. We will find lower bounds for the representation dimension of a larger class of algebras, and more general methods to establish lower bounds for the representation dimension of a given family of algebras.

The first result presented here covers the group algebras of elementary abelian groups. Since group algebras are self-injective, the stable module category, which will be denoted be $k G-\underline{\bmod }$, is a triangulated category. Then we will prove the following:

Theorem 1. Let $k$ be a field of characteristic $p, C_{p}^{n}$ the elementary abelian group of order $p^{n}$. Then

$$
\operatorname{dim} k C_{p}^{n} \underline{-\bmod } \geq n-1 .
$$

It should be noted that Rouquier's analysis of the exterior algebras includes the case $p=2$ of this theorem. One main idea of Rouquier's proof is to use Koszul duality to transfer the problem to a question on coherent sheaves on projective space, and use results from commutative algebra there. In contrast, we will work directly in the module category and show that certain morphisms factor through projective modules, and therefore vanish in the stable category.

By results of Rouquier it follows that the representation dimension of $k C_{p}^{n}$ is at least $n+1$. Generalizing ideas from Rouquier's paper we obtain the same lower bounds for group algebras of groups containing such elementary abelian subgroups, and finally for arbitrary blocks of group algebras: 
Corollary 3.2.4. Let $k$ be a field of characteristic $p$. Let $G$ be a finite group, $B$ a non-semisimple block of $k G$ and $D$ a defect group of $B$. Then

$$
\operatorname{dim} B-\underline{\bmod } \geq p-\operatorname{rank}(D)-1 \quad \text { and } \quad \operatorname{repdim} B \geq p-\operatorname{rank}(D)+1,
$$

where $p-\operatorname{rank}(D)$ denotes the maximal rank of an elementary abelian subgroup of $D$, that is the maximal $r$ such that there is a monomorphism $k C_{p}^{r} \longrightarrow D$.

Since the representation dimension of a self-injective algebra is bounded above by its Loewy length, denoted by LL, we obtain the following corollary, which had been conjectured by Benson.

Corollary 3.2.5 (Benson's conjecture). Let $k$ be a field of characteristic $p$. Let $G$ be a finite group, $B$ a block of $k G$, and $D$ a defect group of $B$. Then

$$
\text { LL } B>p-\operatorname{rank}(D) \text {. }
$$

The second and main result of this thesis is a more general method to establish lower bounds for the representation dimension of classes of algebras. The main ingredients are as follows:

We extend Rouquier's definition of dimension of a triangulated category to arbitrary subcategories of triangulated categories. Looking at the dimension of a module category (as subcategory of its derived category) will allow us to find better lower bounds than by considering only the dimension of the derived category. In many examples we will even be able to show that the representation dimension is strictly larger than the dimension of the derived category. In particular we will be able to improve Krause and Kussin's bound to $N+1$, which will then be shown to be the precise value.

We encode families of modules in lattices, which then automatically also contain some information about the extensions between the members of the families. More precisely, in order to find a lower bound for the representation dimension of a finite dimensional algebra $\Lambda$ over a field $k$, we choose a $\Lambda \otimes_{k} R$ lattice $L$ (where $R$ is a polynomial ring over $k$, or an integral quotient of such a polynomial ring), such that the modules in the family are just the quotients of $L$ modulo the maximal ideals of $R$. This construction yields a functor

$$
L \otimes_{R}-: R-\bmod \longrightarrow \Lambda-\operatorname{Mod}
$$

which is exact and therefore also induces maps between corresponding Extgroups.

These ideas lead up to the following result: 
Theorem (Corollary 5.1.6). Let $L$ be a $\Lambda \otimes_{k} R$-lattice, and $d \in \mathbb{N}$. Assume the set

$$
\left\{\mathfrak{p} \in \operatorname{MaxSpec} R \mid\left(L \otimes_{R}-\right)\left(\operatorname{Ext}_{R}^{d}\left(R_{\mathfrak{p}} \text {-f.l., } R_{\mathfrak{p}} \text {-f.l. }\right)\right) \neq 0\right\}
$$

is Zariski dense in MaxSpec $R$. Then

$$
\operatorname{repdim} \Lambda \geq d+2 \text {. }
$$

We actually prove a refinement of this theorem, which works with complexes of injective modules in the derived category (Theorem 2) and a version which is easier to apply to examples (Theorem 3). It turns out that these theorems provide useful lower bounds for the representation dimension in a variety of situations, in many of which we will see that they are equal or very close to the correct number.

We reprove Rouquier's result on the representation dimension of the exterior algebra of an $N$-dimensional vector space and generalize it to the quotient of the exterior algebra modulo the $L$-th power of the radical (Theorem 7.1.1). For $L \neq N$ we can show that the lower bound we find for the representation dimension is the precise value.

We prove that the representation dimension of $k\left[x_{1}, \ldots, x_{N}\right] /\left(x_{1}, \ldots, x_{N}\right)^{L}$ is at least $\min \{L+1, N+1\}$ (Theorem 7.2.1). For $N \geq L$ we are able to show that this is the correct number. This result carries over to algebras of the form $k Q / I$, with

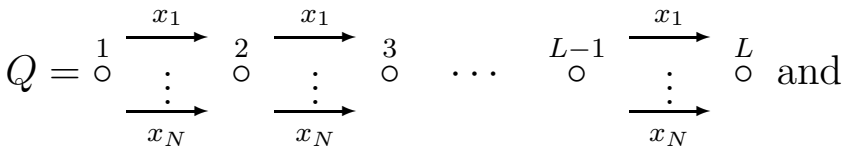

$$
\begin{aligned}
& I=\left(x_{m} x_{n}-x_{n} x_{m} \mid 1 \leq m, n \leq N\right)
\end{aligned}
$$

(Theorem 7.2.6). This generalizes the family considered by Krause and Kussin. Especially we improve the lower bound in their case $(L=N)$ from $N-1$ to $N+1$, and show that this is the precise value.

One advantage of the theorem presented here is, that it is quite well behaved under changes of the algebra. In all previous papers an equivalence of the derived or stable module category to some other triangulated category has been used. In that case one did not automatically get any results for similar algebras. With the method presented here it will usually be possible to transfer results to other algebras with a similar structure (see 5.3 and the examples in Section 7). Especially we will get lower bounds for the representation dimension of algebras depending on parameters in $k$, not just for discrete families (Theorem 7.1.10, Example 7.1.11 and Theorem 7.2.9). 
In the next section we will recall the background of this thesis. First the definition of representation dimension will be examined and some consequences will be discussed. In particular we will give a proof of Auslander's theorem (Corollary 2.1.3), saying that an algebra is of finite representation type if and only if its representation dimension is at most two. Then we will give a few classes of algebras known to have representation dimension three (2.1.4 - 2.1.7).

Next we will recall Rouquier's definition of dimension of a triangulated category, and some properties of this dimension. We will see that the dimension of the derived category (Proposition 2.2.7) and, in the case of a selfinjective algebra, the stable module category (Lemma 2.2.5) provide lower bounds for the representation dimension. Next we will recall a criterion of Rouquier, which helps to establish lower bounds for the dimension of a triangulated category (Lemma 2.2.9). The first new result presented here is Proposition 2.2.16, which is a converse to Rouquier's criterion, and therefore says that it is always possible to establish the optimal lower bound for the dimension of the stable or derived category of a module category by applying this criterion.

In Subsection 2.3 we recall a result from commutative algebra, saying that any coherent sheaf over a suitable scheme is projective in many points (Lemma 2.3.1). This yields a lower bound for the derived category of coherent sheaves (2.3.2). In 2.4 we sketch how this result, together with certain derived equivalences, gives the lower bounds in Rouquier's (Theorem 2.4.1) and in Krause and Kussin's (Theorem 2.4.3) examples.

In Section 3 we study the group algebras of elementary abelian groups. We show that the rank of the group minus one is a lower bound for the dimension of the stable module category. This is done by applying the criterion of Rouquier. To be able to apply this criterion, we have to find for any given module $M$ a sequence of morphisms, such that the composition of the morphisms does not vanish, but every one of the morphisms is annihilated by all morphisms from $M$. We first assume that we are dealing with an algebra over an infinite field, and explicitly construct the desired sequence of morphisms (Propositions 3.1.3 and 3.1.4). Then we carry over this result to algebras over finite fields by using a suitable finite separable extension of the field.

This implies that the representation dimension of such an algebra is at least the rank of the group plus one. In the second subsection we see that these results can be carried over to arbitrary blocks of group algebras, provided the defect group has elementary abelian subgroups of sufficiently large rank. Finally this leads to a proof of the conjecture of Benson stated above. 
We use the fourth section to generalize Rouquier's definition of dimension of a triangulated category to arbitrary subcategories of triangulated categories. In particular, since the module category is a subcategory of its derived category, we obtain a concept of dimension of a module category. It turns out that the representation dimension of an algebra is bounded below by the dimension of the module category plus two. Next we adjust Rouquier's criterion to be able to use it to find lower bounds for the dimension of the module category (Proposition 4.8). Finally we show that in the inequalities used to estimate the representation dimension by the dimension of the module category equality holds if the numbers occurring are sufficiently small (Corollaries 4.11 and 4.15). This is an advantage compared to the inequality using the dimension of the derived category, since there it is not known if equality can ever hold.

In Section 5 we prove a criterion (Theorem 2) which helps find lower bounds for the dimension of a given module category, and therefore also for the representation dimension of the corresponding algebra. To do so, we construct a pair of adjoint functors between the (derived category of) the module category we are interested in, and the (derived category of) finite length modules over a commutative ring. For the latter we can apply results from commutative algebra, and then transfer the results to our original category with the help of these functors.

In the second subsection we further investigate one special case, in which the calculations are easier. Here we get a simplified version of the general criterion (Theorem 3).

In the last subsection we will see that the criterion is well behaved under certain coverings of algebras. This means that when we apply the criterion to some algebra we can sometimes automatically get results on many other algebras (we will see examples for this in Section 7).

The sixth section is devoted to Iyama's general upper bound for the representation dimension. We start by stating his theorem (Theorem 6.1) and giving Iyama's proof. Then we prove two corollaries (6.2 and 6.3). The first one is suitable for applying to algebras which have a quiver similar to the one of the algebra investigated by Krause and Kussin. The second one can be used to treat local algebras, such as quotients of the exterior algebras.

In Section 7 we show how the results from Sections 5 and 6 can be applied. First we look at algebras related to the exterior algebras treated by Rouquier. We reprove his result, and generalize it to quotients of the exterior algebras 
modulo some power of the radical. We also obtain a result on a covering of finite global dimension (Theorem 7.1.8). Finally we also obtain results on many deformations of these algebras (Theorem 7.1.10 and Example 7.1.11). This is the first instance of a lower bound for the representation dimension of the members of a family of algebras parametrized by (many) elements of the base field, not just by one discrete parameter.

Next we consider the quotients of polynomial rings modulo some power of the ideal generated by the variables. In many cases we are able to determine the various dimensions (Theorem 7.2.1). This also yields a general upper lower bound for the representation dimension of quotients of polynomial rings (Theorem 4). As for exterior algebras, we consider coverings. Here it turns out that these coverings include the algebras studied by Krause and Kussin. Their lower bound is improved by two, and it is shown that the new lower bound is the exact value.

Finally, we give two more examples of algebras of representation dimension four, to indicate that the criterion for lower bounds is quite generally applicable.

In the eighth and final section we list some questions which remained open, or which came up as a consequence of the results presented here.

Acknowledgements. I am very grateful to my supervisor Steffen Koenig for suggesting this subject and for many helpful and encouraging discussions. Further I wish to thank Osamu Iyama and Idun Reiten for interesting comments and discussions. 


\section{Background}

In this section we recapitulate what had already been known about lower bounds for Auslander's representation dimension before the work on this thesis began. Most of what is said here can be found in the papers of Rouquier $[15,16]$ and Krause and Kussin [12]. However, some side results seem to be new (Lemma 2.2.4 and Proposition 2.2.16).

\subsection{Auslander's representation dimension}

Auslander introduced the notion of representation dimension of a finite dimensional algebra in his Queen Mary College notes [2] in 1971. He showed that the representation dimension of an algebra is at most two if and only if the algebra is of finite representation type (see Corollary 2.1.3 below). This led him to the expectation, that the representation dimension should measure how far a representation infinite algebra is from having finite representation type.

By a finite dimensional algebra we mean an associative algebra which is finite dimensional over some base field. This base field will always be denoted by $k$.

\section{Definition and basic properties}

For a ring $R$, we will denote the category of left $R$-modules by $R$-Mod, and the subcategory of finitely presented modules by $R$-mod. Recall that the global dimension of $R$, denoted by gld $R$, is the maximum of the projective dimensions of the modules.

2.1.1 Definition (Auslander [2]). Let $\Lambda$ be a finite dimensional algebra. Then the representation dimension of $\Lambda$ is defined to be

$$
\begin{array}{r}
\operatorname{repdim} \Lambda=\min \left\{\operatorname{gld} \operatorname{End}_{\Lambda}(M) \mid M \in \Lambda-\bmod \right. \text { generates and } \\
\text { cogenerates } \Lambda \text {-mod }\}
\end{array}
$$

A module $M$ realizing this minimum will be called Auslander generator.

In many situations we will know the module category of $\Lambda$ better than the one of $\operatorname{End}_{\Lambda}(M)$. Then the following lemma will help us to understand the representation dimension.

2.1.2 Lemma (Erdmann, Holm, Iyama, Schroer [8, Lemma 2.1]). Let $\Lambda$ be a non-semisimple algebra, let $M \in \Lambda$-mod be a generator and cogenerator, $n \in \mathbb{N}$. Then the following are equivalent: 
1. $\operatorname{gld} \operatorname{End}_{\Lambda}(M)=n$

2. For every $N \in \Lambda$-mod there is an exact sequence

$$
0 \longrightarrow M_{n-2} \longrightarrow \cdots \longrightarrow M_{0} \longrightarrow N \longrightarrow 0,
$$

such that the induced sequence

$$
0 \longrightarrow \operatorname{Hom}_{\Lambda}\left(M, M_{n-2}\right) \longrightarrow \cdots \longrightarrow \operatorname{Hom}_{\Lambda}(M, N) \longrightarrow 0
$$

is also exact.

Such a sequence will be called a (universal) $M$-resolution of $N$.

This means, that the representation dimension of a non-semisimple algebra can also be defined to be the minimal $n$, such that there is a generator cogenerator $M$ having the property that every module has a universal $M$ resolution of length at most $n-2$.

Proof. Let $N \in \Lambda$-mod. Since $M$ is a cogenerator there is an exact sequence $N \longrightarrow M_{1} \longrightarrow M_{0}$ with $M_{i} \in$ add $M$. Then the sequence

$$
\operatorname{Hom}_{\Lambda}(M, N) \longrightarrow \operatorname{Hom}_{\Lambda}\left(M, M_{1}\right) \longrightarrow \operatorname{Hom}_{\Lambda}\left(M, M_{0}\right)
$$

is also exact. We denote the cokernel of the right morphism by $H_{N}$. Now the sequence

$$
\begin{aligned}
& \operatorname{Hom}_{\Lambda}\left(M, M_{n}\right) \longrightarrow \cdots \longrightarrow \operatorname{Hom}_{\Lambda}\left(M, M_{2}\right) \longrightarrow \\
& \longrightarrow \operatorname{Hom}_{\Lambda}\left(M, M_{1}\right) \longrightarrow \operatorname{Hom}_{\Lambda}\left(M, M_{0}\right) \longrightarrow H_{N}
\end{aligned}
$$

is a projective resolution of the $\operatorname{End}_{\Lambda}(M)$-module $H_{N}$ if and only if

$$
M_{n} \longrightarrow \cdots \longrightarrow M_{2} \longrightarrow N
$$

is a universal $M$-resolution of $N$.

With this lemma Auslander's result follows immediately.

2.1.3 Corollary (Auslander). Let $\Lambda$ be a finite dimensional algebra. Then $\Lambda$ is representation finite if and only if $\operatorname{repdim} \Lambda \leq 2$.

Proof. If $\Lambda$ is semisimple, then it is representation finite and $\operatorname{repdim} \Lambda=0$, so the claim holds.

Assume $\Lambda$ is not semisimple but of finite representation type. Then let $M$ be an additive generator of $\Lambda$-mod. Now any $\Lambda$-module has a universal $M$-resolution of length 0 (namely $M_{0}$ is isomorphic to the module), so by Lemma 2.1.2 we have gld $\operatorname{End}_{\Lambda}(M)=2$.

Now assume $\Lambda$ is not of finite representation type, and $M \in \Lambda$-mod is a generator and a cogenerator. Then there is an $N \in \Lambda-\bmod \backslash$ add $M$. The universal $M$-resolution of this module $N$ has length at least 1 , so by Lemma 2.1.2 it follows that gld $\operatorname{End}_{\Lambda}(M) \geq 3$. 


\section{Upper bounds}

To establish an upper bound for the representation dimension of a given algebra $\Lambda$, one has to find an $M \in \Lambda$-mod such that the global dimension of $\operatorname{End}_{\Lambda}(M)$ is the bound. It was not known, until Iyama gave an explicit construction, which will be presented in Section 6 here, whether this is always possible. However, there is a number of classes of algebras for which an $M$ with gld $\operatorname{End}_{\Lambda}(M)=3$ has been constructed. Here we give some examples of such. Moreover we recall a result of Auslander providing an upper bound for the representation dimension of self-injective algebras (Lemma 2.1.8).

2.1.4 Lemma (Auslander). Let $\Lambda$ be a hereditary finite dimensional algebra. Then repdim $\Lambda \leq 3$.

Proof. Take $M=\Lambda \oplus \Lambda^{*}$.

For a finite dimensional algebra $\Lambda$, we denote by $\operatorname{Rad} \Lambda$ the Jacobson radical of $\Lambda$. The Loewy length of a $\Lambda$-modules $M$, denoted by LL $M$, is the minimal $n$ such that $\operatorname{Rad}^{n} M=0$.

2.1.5 Lemma (Auslander). Let $\Lambda$ be a finite dimensional algebra with $\operatorname{LL} \Lambda=2$. Then $\operatorname{repdim} \Lambda \leq 3$.

Proof. Take $M=\Lambda \oplus \Lambda^{*} \oplus \Lambda / \operatorname{Rad} \Lambda$.

2.1.6 Theorem ([8, Corollary 1.3]). Let $\Lambda$ be a special biserial algebra. Then $\operatorname{repdim} \Lambda \leq 3$.

2.1.7 Theorem ([1, Theorem 2.3]). Let $\Lambda$ be a tilted algebra. Then repdim $\Lambda \leq 3$.

Proof. See [1, Theorem 2.3]. They take $M=\Lambda \oplus \Lambda^{*} \oplus T$, where $T$ is the direct sum over the indecomposable modules in a complete slice.

2.1.8 Lemma ([3, I, Theorem 10.3]). Let $\Lambda$ be self-injective. Then

$$
\operatorname{repdim} \Lambda \leq \operatorname{LL} \Lambda \text {. }
$$

Proof. Take $M=\bigoplus_{i} \Lambda / \operatorname{Rad}^{i} \Lambda$. Let us denote for a moment the kernel of the universal $M$-cover (that is the first morphism of the minimal universal $M$-resolution) of a module $N$ by $\Omega_{M} N$. Now note that in every step of the resolution, the Loewy length of the module decreases by at least one, that means $\operatorname{LL} \Omega_{M}^{i+1} N \leq \operatorname{LL} \Omega_{M}^{i} N-1$. If $N$ is projective then it is in add $M$, therefore we may assume that $\operatorname{LL} N<\operatorname{LL} \Lambda$. Putting this together we find $\operatorname{LL} \Omega_{M}^{\mathrm{LL} \Lambda-1} N=0$, so $\Omega_{M}^{\mathrm{LL} \Lambda-1} N=0$. Therefore repdim $\Lambda \leq \operatorname{LL} \Lambda$ as claimed. 


\subsection{The dimension of a triangulated category}

The notion of dimension of a triangulated category has been introduced by Rouquier in [15]. He showed how it can be used to establish lower bounds for the representation dimension.

\section{Definition and basic properties}

Let $\mathcal{T}$ be a triangulated category, $\mathcal{I} \subset$ ob $\mathcal{T}$. Then let $\langle\mathcal{I}\rangle$ be the full subcategory of $\mathcal{T}$ of all direct summands of finite direct sums of shifts of objects in $\mathcal{I}$. For two subclasses $\mathcal{I}_{1}, \mathcal{I}_{2} \subset$ o6 $\mathcal{T}$ let $\mathcal{I}_{1} * \mathcal{I}_{2}$ be the full subcategory of all extensions between them, that is the objects of $\mathcal{I}_{1} * \mathcal{I}_{2}$ are exactly the $M$, such that there is a distinguished triangle $M_{1} \longrightarrow M \longrightarrow M_{2} \longrightarrow M_{1}[1]$ in $\mathcal{T}$ with $M_{i} \in \mathcal{I}_{i}$. Now set

$$
\mathcal{I}_{1} \diamond \mathcal{I}_{2}=\left\langle\mathcal{I}_{1} * \mathcal{I}_{2}\right\rangle
$$

and

$$
\begin{aligned}
& \langle\mathcal{I}\rangle_{0}=0, \\
& \langle\mathcal{I}\rangle_{1}=\langle\mathcal{I}\rangle \\
& \langle\mathcal{I}\rangle_{i+1}=\langle\mathcal{I}\rangle_{i} \diamond\langle\mathcal{I}\rangle .
\end{aligned}
$$

2.2.1 Definition (Rouquier). The dimension of a triangulated category $\mathcal{T}$ is the minimal $d$ such that there is an object $M \in \mathcal{T}$ with $\mathcal{T}=\langle M\rangle_{d+1}$. If no such $M$ exists for any $d$, then we set $\operatorname{dim} \mathcal{T}=\infty$.

The following lemma is an immediate consequence of this definition:

2.2.2 Lemma ([15, Lemma 3.4]). Let $\mathcal{S} \stackrel{\mathrm{F}}{\longrightarrow} \mathcal{T}$ be a triangle functor between two triangulated categories. Assume any object in $\mathcal{T}$ is a direct summand of an object in the image of $\mathrm{F}$. Then

$$
\operatorname{dim} \mathcal{T} \leq \operatorname{dim} \mathcal{S}
$$

As for the representation dimension, upper bounds for the dimension of a triangulated category can be established by explicitly giving an $M$ which generates the category in the desired number of steps. We give two examples of this technique.

The following is a slight extension of [12, Lemma 2.5].

2.2.3 Lemma. Let $\Lambda$ be a finite dimensional algebra. Let $X \in D^{b}(\Lambda$-mod) be a complex such that all homology modules have projective dimension at most $n$. Then $X \in\langle\Lambda\rangle_{n+1} \subseteq D^{b}(\Lambda$-mod $)$.

In particular

$$
\operatorname{dim} D^{b}(\Lambda-\bmod ) \leq \operatorname{gld} \Lambda
$$


Proof. We will prove this by induction on $n$. For $n=0$ the claim clearly holds.

Let $X=\left(X^{i}\right), M^{i}$ be the images of differential $d^{i}$, and $p^{i}$ and $j^{i}$ as indicated in the following diagram.

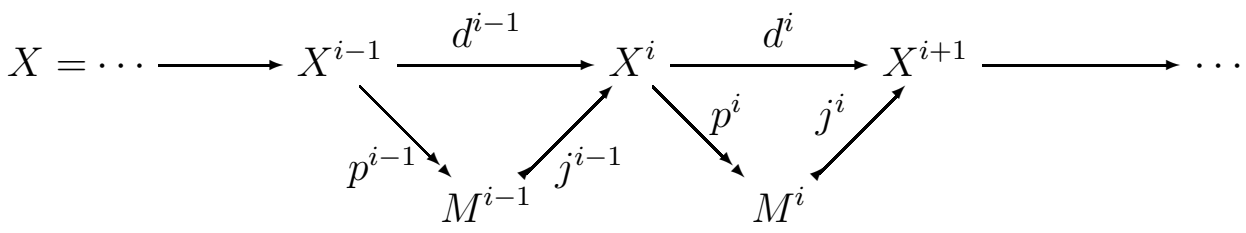

Then the homology modules are $\operatorname{Ker} p^{i} / M^{i-1}$. Let $P^{i} \longrightarrow \operatorname{Ker} p^{i} / M^{i-1}$ be projective covers. Let $\varphi_{0}^{i}$ be the compositions of these projective covers with the embeddings $\operatorname{Ker} p^{i} / M^{i-1} \longrightarrow X^{i} / M^{i-1}$. Then clearly $\operatorname{Ker} p^{i} / M^{i-1}=$ $\operatorname{Im} \varphi_{0}^{i}$. Since the $P^{i}$ are projective the $\varphi_{0}^{i}$ induce maps $\varphi^{i}: P^{i} \longrightarrow X^{i}$. Let $P=\left(P^{i}\right)$ with zero differential. Then $P \in\langle\Lambda\rangle$ and $\varphi$ is a map of complexes. Now we consider the mapping cone of $\varphi$. We will show that its homology modules are exactly the syzygies of the homology modules of $X$. Then clearly the claim of the lemma follows by induction.

Up to isomorphism, the mapping cone is

$$
\cdots \longrightarrow P^{i} \oplus X^{i-1} \stackrel{\left(\begin{array}{cc}
0 & \varphi^{i} \\
0 & d^{i-1}
\end{array}\right)}{\longrightarrow} P^{i+1} \oplus X^{i} \stackrel{\left(\begin{array}{cc}
0 & \varphi^{i+1} \\
0 & d^{i}
\end{array}\right)}{\longrightarrow} P^{i+2} \oplus X^{i+1} \longrightarrow \cdots
$$

The image of $\left(\begin{array}{cc}0 & \varphi^{i} \\ 0 & d^{i-1}\end{array}\right)$ is $\operatorname{Ker} p^{i}$, so the homology in position $i$ is just the kernel $K$ of the map $P^{i+1} \oplus M^{i} \stackrel{\left(\begin{array}{c}\varphi^{i+1} \\ j^{i}\end{array}\right)}{\longrightarrow} X^{i+1}$, which is the pullback in the following diagram with short exact rows.

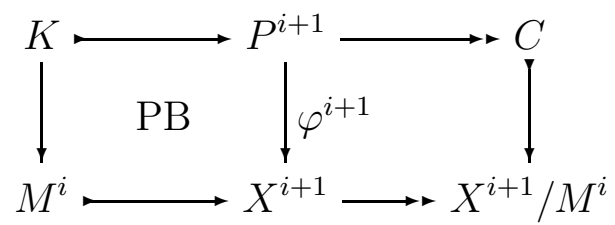

Since the left square is a pullback the right vertical morphism is mono. Therefore $C$ is the image of the map $\varphi_{0}^{i+1}$, which is the homology module $\operatorname{Ker} p^{i+1} / M^{i}$. Therefore $K$ is indeed the syzygy of a homology module of $X$.

2.2.4 Lemma. Let $\Lambda$ be a finite dimensional algebra. Let $X=\left(X^{i}\right)_{i \in \mathbb{Z}}$ be a complex of $\Lambda$-modules, such that all $X^{i}$ have Loewy length at most $n$. Then $X \in\langle\Lambda / J \Lambda\rangle_{n} \subseteq D^{b}(\Lambda-\bmod )$.

In particular

$$
\operatorname{dim} D^{b}(\Lambda-\bmod ) \leq \operatorname{LL} \Lambda-1
$$


Proof. We use induction on $n$. For $n=1$ the claim clearly holds. Assume $n>1$ and consider the following short exact sequence of complexes.

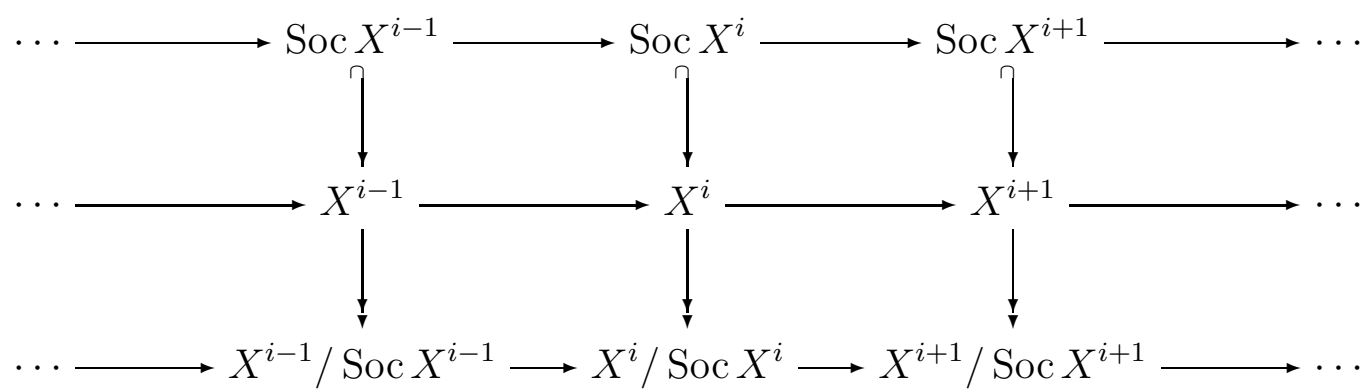

This short exact sequence induces a triangle in $D^{b}(\Lambda-\bmod )$. The top row is in $\langle\Lambda / J \Lambda\rangle_{1}$, the bottom row is in $\langle\Lambda / J \Lambda\rangle_{n-1}$ by induction. The claim of the lemma now follows immediately from the definition of $\langle\Lambda / J \Lambda\rangle_{n}$.

\section{Connections to representation dimension}

We denote the bounded derived category of an abelian category $\mathcal{A}$ by $D^{b}(\mathcal{A})$. For $\mathcal{A}=\Lambda$-mod, we let $\Lambda$-perf be the full subcategory whose objects are finite complexes of projective $\Lambda$-modules.

For a self-injective algebra $\Lambda$, the stable module category is denoted by $\Lambda$-mod. By [14, Theorem 2.1], in that situation we have $\Lambda$ - $\underline{\bmod }=$ $D^{b}(\Lambda-\bmod ) / \Lambda$-perf.

2.2.5 Lemma ([16, part of Proposition 3.6]). Let $\Lambda$ be a non-semisimple finite dimensional algebra. Then

$$
\operatorname{repdim} \Lambda \geq \operatorname{dim} D^{b}(\Lambda-\bmod ) / \Lambda-\operatorname{perf}+2 .
$$

In particular, if $\Lambda$ is self-injective, then

$$
\operatorname{repdim} \Lambda \geq \operatorname{dim} \Lambda \underline{\bmod }+2 \text {. }
$$

Proof. Let $M$ be an Auslander generator. By Lemma 2.1.2, any module $N$ has a universal $M$-resolution of length at most repdim $\Lambda-2$. Since short exact sequences in $\Lambda$-mod become triangles in $D^{b}(\Lambda$-mod $) / \Lambda$-perf, any $N$ having an $M$ resolution of length repdim $\Lambda-2$ is contained in $\langle M\rangle_{\text {repdim } \Lambda-1}$. Therefore $\operatorname{dim} D^{b}(\Lambda$-mod $) / \Lambda$-perf $\leq \operatorname{repdim} \Lambda-2$.

2.2.6 Remark. Note that for $\Lambda$ an algebra of finite global dimension

$$
D^{b}(\Lambda-\bmod )=\Lambda \text {-perf }
$$

so the lemma above does not help to determine the representation dimension in these cases. 
Now we follow Krause and Kussin [12], to see that the representation dimension is also larger than or equal to the dimension of the derived category.

2.2.7 Proposition ([12, Corollary 3.6]). Let $\Lambda$ be a finite dimensional algebra. Then

$$
\operatorname{repdim} \Lambda \geq \operatorname{dim} D^{b}(\Lambda-\bmod ) \text {. }
$$

Proof. Let $M$ be a module realizing the minimal $n$ in the definition of representation dimension. We may assume that $n$ is finite (either because Iyama $[10,11]$ has shown that the representation dimension is always finite (see Theorem 6.1), or because the claim of the Corollary is trivial otherwise). Let $\Gamma=\operatorname{End}_{\Lambda}(M)$. Then add $M \cong \Gamma$-proj and therefore $K^{b}(\Gamma$-proj $) \cong K^{b}($ add $M)$, where $K^{b}$ denotes the bounded homotopy category. By Lemma 2.1.2 any $\Lambda$-module has a finite resolution by objects from add $M$. Therefore the functor $K^{b}($ add $M) \longrightarrow D^{b}(\Lambda$-mod) has dense image, and so does the composition

$$
D^{b}(\Gamma-\bmod ) \cong K^{b}(\Gamma-\operatorname{proj}) \cong K^{b}(\operatorname{add} M) \longrightarrow D^{b}(\Lambda-\bmod ) .
$$

By Lemma 4.2 we have $\operatorname{dim} D^{b}(\Lambda$-mod $) \leq \operatorname{dim} D^{b}(\Gamma-\bmod )$. By Lemma 2.2.3 the latter is at most $\operatorname{gld} \Gamma=\operatorname{repdim} \Lambda$.

2.2.8 Remark. It is not known whether there is a non-semisimple algebra for which equality holds in Proposition 2.2.7.

\section{Lower bounds for the dimension of a triangulated category}

Clearly the inequalities above are of no particular interest, unless we can find lower bounds for the dimension of one of the triangulated categories that come with a finite dimensional algebra. Rouquier did so by using the following lemma.

2.2.9 Lemma ([12, Lemma 2.3] and [15, Lemma 4.11]). Let $\mathcal{T}$ be a triangulated category and let

$$
H_{1} \stackrel{f_{1}}{\longrightarrow} H_{2} \stackrel{f_{2}}{\longrightarrow} \cdots \stackrel{f_{n-1}}{\longrightarrow} H_{n-1} \stackrel{f_{n-1}}{\longrightarrow} H_{n}
$$

be a sequence of morphisms between cohomological functors $\mathcal{T}^{\mathrm{op}} \longrightarrow \mathrm{Ab}$. For every $i$, let $\mathcal{I}_{i}$ be a subcategory of $\mathcal{T}$ closed under shifts and on which $f_{i}$ vanishes. Then $f_{1} \cdots f_{n-1}$ vanishes on $\mathcal{I}_{1} \diamond \cdots \diamond \mathcal{I}_{n-1}$.

Proof. We will prove the lemma by induction on $n$. For $n=0$ there is nothing to show. For $n=2$ we have maps $f_{1}: H_{1} \longrightarrow H_{2}$ and $f_{2}: H_{2} \longrightarrow H_{3}$ which vanish on $\mathcal{I}_{1}$ and $\mathcal{I}_{2}$ respectively. Assume $M \in \mathcal{I}_{1} \diamond \mathcal{I}_{2}$. We may assume that 
there is a triangle $M_{1} \longrightarrow M \longrightarrow M_{2} \longrightarrow M_{1}[1]$ with $M_{i} \in \mathcal{I}_{i}$. We have to show that $f_{1} f_{2}$ vanishes on $M$. Since the $H_{i}$ are cohomological functors, they turn the triangle into the long exact sequences which from the rows of the following diagram.

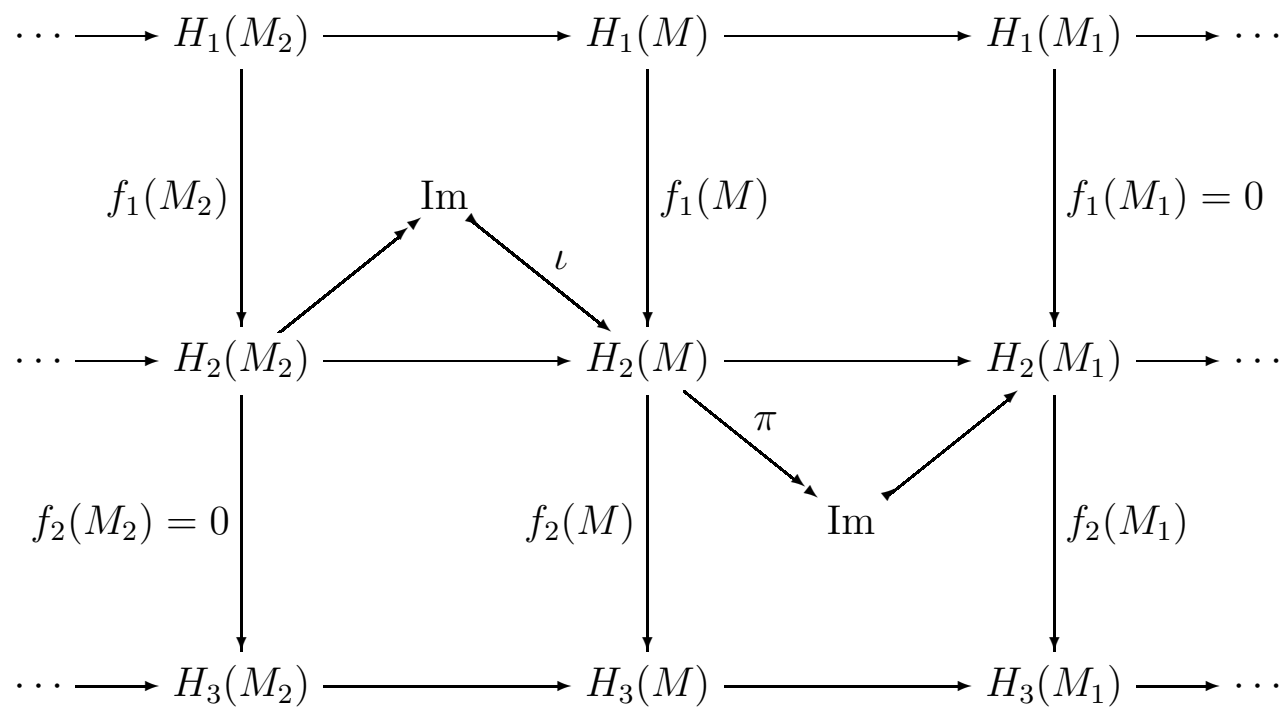

We add the images of the morphisms in the central sequence as indicated in the diagram. Since $f_{1}\left(M_{1}\right)$ vanishes, $f_{1}(M)$ factors through $\iota$. Dually $f_{2}(M)$ factors through $\pi$. But $\iota \pi=0$, and therefore also $\left(f_{1} f_{2}\right)(M)=0$.

Now assume $n>2$ and the claim holds for all $n^{\prime}<n$. Especially $f_{1} \cdots f_{n-2}$ vanishes on $\mathcal{I}_{1} \diamond \cdots \diamond \mathcal{I}_{n-2}$. Now apply the case $n=2$ th the two morphisms $f_{1} \cdots f_{n-2}$ and $f_{n-1}$ which vanish on the subcategories $\mathcal{I}_{1} \diamond \cdots \diamond \mathcal{I}_{n-2}$ and $\mathcal{I}_{n-1}$ respectively.

We will apply the following instance of that lemma.

2.2.10 Corollary. Let $\mathcal{T}$ be a triangulated category, $M \in$ ob $\mathcal{T}$. Let $n \in \mathbb{N}$ such that there is a sequence of morphisms in $\mathcal{T}$

$$
N_{1} \stackrel{f_{1}}{\longrightarrow} N_{2} \stackrel{f_{2}}{\longrightarrow} \cdots \stackrel{f_{n-1}}{\longrightarrow} N_{n-1} \stackrel{f_{n-1}}{\longrightarrow} N_{n}
$$

such that

1. the composition $f_{1} \cdots f_{n}$ does not vanish, and

2. $\operatorname{Hom}_{\mathcal{T}}\left(M, N_{i}\right) \cdot f_{i}=0 \quad \forall i$.

Then $N_{1} \notin\langle M\rangle_{n-1}$.

In particular, if such a sequence exists for any $M$, then $\operatorname{dim} \mathcal{T} \geq n-1$. 
Proof. Set $\mathcal{I}_{i}=\langle M\rangle$ and $H_{i}=\operatorname{Hom}_{\mathcal{T}}\left(-, N_{i}\right)$ in Lemma 2.2.9 above.

2.2.11 Corollary. Let $\Lambda$ be a finite dimensional algebra, gld $\Lambda=n<\infty$. Then $\langle\Lambda\rangle_{n} \subsetneq D^{b}(\Lambda-\bmod )=\langle\Lambda\rangle_{n+1}$.

Proof. The equality is Lemma 2.2.3.

To see that $\langle\Lambda\rangle_{n}$ is not the entire derived category choose a module $X$ with $\operatorname{pd} X=n$. Let its projective resolution be

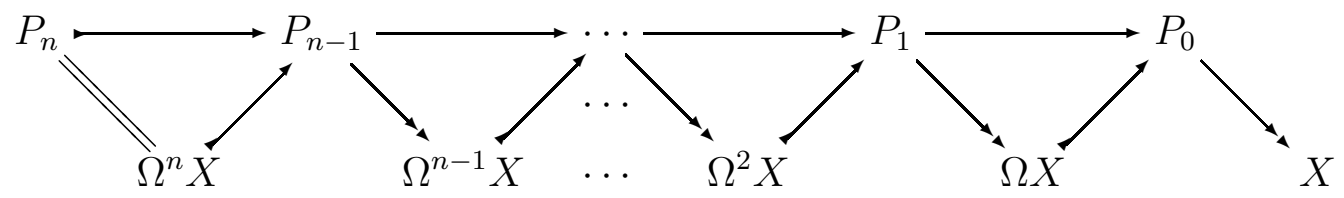

The 1-extensions $\Omega^{i} X \longrightarrow P_{i-1} \longrightarrow \Omega^{i-1} X$ correspond to morphisms $\Omega^{i-1} X \longrightarrow\left(\Omega^{i} X\right)[1]$ in the derived category. Clearly all homomorphisms from shifts of $\Lambda$ are annihilated by these maps. Therefore, by Corollary 2.2.10, $X \notin\langle\Lambda\rangle_{n}$, so $\langle\Lambda\rangle_{n}$ cannot be the entire derived category.

We will use the rest of this section to prove a converse of Lemma 2.2.9 in the situations we are most interested in. This will not help to establish better lower bounds, but since most arguments will consist of a series of inequalities is is natural to ask if and how much we loose in each step. This converse seems to be new.

2.2.12 Setup. We assume that we have two triangulated categories $\mathcal{T} \subset \mathcal{S}$ with the following properties:

1. $\mathcal{S}$ is cocomplete, that means $\mathcal{S}$ has arbitrary (set indexed) coproducts, and

2. for any $T, T_{i} \in \mathcal{T}$, any morphism $T \longrightarrow \coprod_{i} T_{i}$ factors through a finite subcoproduct:

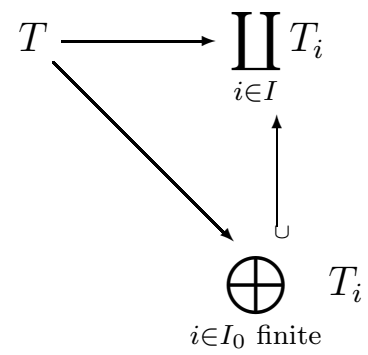

Let us first see that in the most important situations here the assumptions above are satisfied.

2.2.13 Lemma. Let $\mathcal{T}=D^{b}(\Lambda$-mod) and $\mathcal{S}=D(\Lambda$-Mod $)$ or $\mathcal{T}=\Lambda$ - $\underline{\bmod }$ and $\mathcal{S}=\Lambda$-Mod. Then 1. and 2. hold for $\mathcal{T}$ and $\mathcal{S}$. 
Proof. In both cases it is clear that $\mathcal{S}$ is cocomplete.

Let us first assume that we have $\mathcal{T}=\Lambda$ - $\underline{\bmod }$ and $\mathcal{S}=\Lambda$-Mod. Let $T, T_{i} \in \Lambda$ - $\underline{\bmod }$ and $f: T \longrightarrow \coprod T_{i}$. We choose representatives in $\Lambda$-mod which we will also denote by $T, T_{i}$ and $f$. Let $t_{1}, \ldots, t_{r}$ generate $T$. The images $t_{1} f, \ldots, t_{r} f$ can only have a nonzero component in finitely many of the direct summands of $\amalg T_{i}$.

Now assume $\mathcal{T}=D^{b}(\Lambda-\bmod )$ and $\mathcal{S}=D(\Lambda-\mathrm{Mod})$. Let $T, T_{i}$ and $f$ as in 2. We replace $T$ and the $T_{i}$ by complexes of finitely generated injective modules. Without loss of generality we may assume that $H^{i}(T)=0 \forall i \geq 0$ (otherwise shift the whole situation). As before, we can see that the images of the morphisms $f_{i}, i \leq 0$ (that is the part of $f$ in degree $i$ ) only have components in finitely many of the direct summands of $\amalg T_{i}$. Let $i_{0}$ not be among these. Then the morphism $T \longrightarrow T_{i_{0}}$ is zero, since one can (inductively from left to right) construct a homotopy.

For a subcategory $\mathcal{I} \subseteq \mathcal{T}$ we denote by ads $\mathcal{I}$ the full subcategory of $\mathcal{S}$, whose objects are direct summands of arbitrary direct sums of objects in $\mathcal{I}$.

2.2.14 Lemma (idea taken from [15, Corollary 3.14]). Let $\mathcal{T} \subset \mathcal{S}$ be triangulated categories as in Setup 2.2.12, $\mathcal{I}_{i}$ subcategories of $\mathcal{T}$. Let $T \in$ ${ }_{06} \mathcal{T}, I \in\left\langle\operatorname{ads} \mathcal{I}_{1}\right\rangle \diamond \cdots \diamond\left\langle\operatorname{ads} \mathcal{I}_{n}\right\rangle$. Then any morphism $T \longrightarrow I$ factors through $\left\langle\mathcal{I}_{1}\right\rangle \diamond \cdots \diamond\left\langle\mathcal{I}_{n}\right\rangle$.

Proof. We will proceed by induction on $n$. In case $n=1$ the claim holds by assumption 2 of Setup 2.2.12.

Let $I_{n} \in\left\langle\operatorname{ads} \mathcal{I}_{1}\right\rangle \diamond \cdots \diamond\left\langle\operatorname{ads} \mathcal{I}_{n}\right\rangle$. We may assume that $I_{n} \in\left(\left\langle\operatorname{ads} \mathcal{I}_{1}\right\rangle \diamond\right.$ $\left.\cdots \diamond\left\langle\operatorname{ads} \mathcal{I}_{n-1}\right\rangle\right) *\left\langle\operatorname{ads} \mathcal{I}_{n}\right\rangle$. That means there is a triangle

$$
I_{n-1} \longrightarrow I_{n} \longrightarrow I_{1} \longrightarrow I_{n-1}[1]
$$

with $I_{n-1} \in\left\langle\operatorname{ads} \mathcal{I}_{1}\right\rangle \diamond \cdots \diamond\left\langle\operatorname{ads} \mathcal{I}_{n-1}\right\rangle$ and $I_{1} \in\left\langle\operatorname{ads} \mathcal{I}_{n}\right\rangle$. By assumption, the composition $T \longrightarrow I_{n} \longrightarrow I_{1}$ factors through $I_{1}^{0} \in\left\langle\mathcal{I}_{n}\right\rangle$ as indicated in the following diagram.

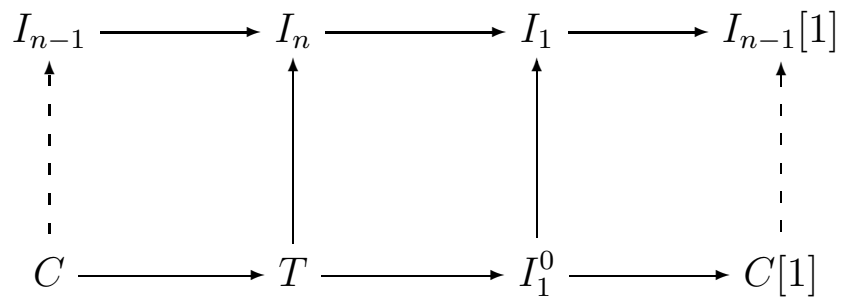

Here $C$ is the cocone of the map $T \longrightarrow I_{1}^{0}$, and the dashed arrow exist since we are working in a triangulated category. 
Now, inductive assumption, the map $C \longrightarrow I_{n-1}$ factors through an object $I_{n-1}^{0} \in\left\langle\mathcal{I}_{1}\right\rangle \diamond \cdots \diamond\left\langle\mathcal{I}_{n-1}\right\rangle$. Let $I_{n}^{0}$ be the cone of the composition $I_{1}^{0}[-1] \longrightarrow C \longrightarrow I_{n-1}^{0}$. Then $I_{n}^{0} \in\left\langle\mathcal{I}_{1}\right\rangle \diamond \cdots \diamond\left\langle\mathcal{I}_{n}\right\rangle$ and the following diagram commutes.

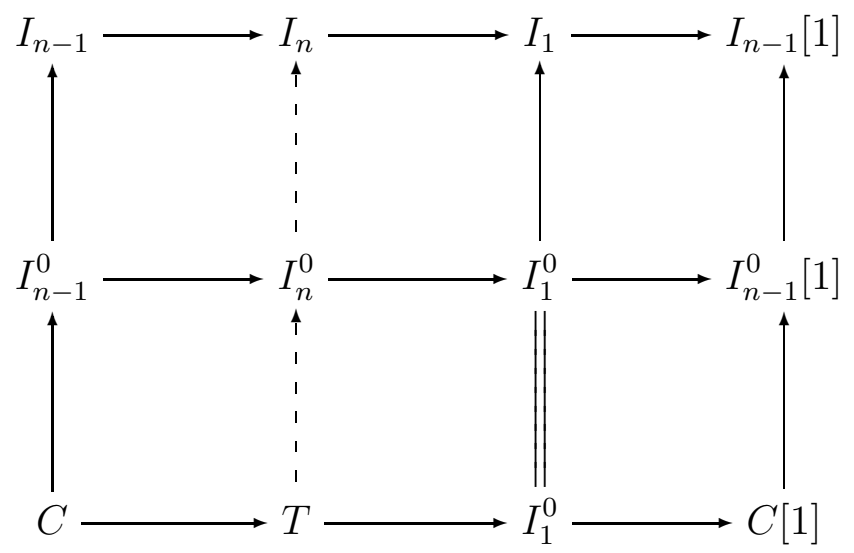

Now the difference between the dashed composition and our original morphism factors through $I_{1}^{0}$, so the claim holds.

2.2.15 Corollary. Let $\mathcal{T} \subset \mathcal{S}$ be triangulated categories as in Setup 2.2.12, $\mathcal{I}_{i}$ subcategories of $\mathcal{T}$. Then

$$
\left(\left\langle\operatorname{ads} \mathcal{I}_{1}\right\rangle \diamond \cdots \diamond\left\langle\operatorname{ads} \mathcal{I}_{n}\right\rangle\right) \cap \mathcal{T}=\left\langle\mathcal{I}_{1}\right\rangle \diamond \cdots \diamond\left\langle\mathcal{I}_{n}\right\rangle .
$$

Especially, for $\mathcal{I} \subset \mathcal{T}$ and $n \in \mathbb{N}$,

$$
\langle\operatorname{ads} \mathcal{I}\rangle_{n} \cap \mathcal{T}=\langle\mathcal{I}\rangle_{n}
$$

Proof. Let $T \in\left(\left\langle\operatorname{ads} \mathcal{I}_{1}\right\rangle \diamond \cdots \diamond\left\langle\operatorname{ads} \mathcal{I}_{n}\right\rangle\right) \cap \mathcal{I}$. Then $1_{T}$ factors through an object in $\left\langle\mathcal{I}_{1}\right\rangle \diamond \cdots \diamond\left\langle\mathcal{I}_{n}\right\rangle$.

Now we are ready to prove the promised converse to Lemma 2.2.9.

We call a category $\mathcal{I}$ skeletally small if the isomorphism classes of objects form a set.

2.2.16 Proposition. Let $\mathcal{T} \subset \mathcal{S}$ be triangulated categories satisfying the assumptions of Setup 2.2.12. Let $\mathcal{I}_{i}(1 \leq i \leq n)$ be skeletally small subcategories of $\mathcal{T}$ closed under shifts. Let $X \in \mathrm{ob} \mathcal{T} \backslash$ ob $\mathcal{I}_{1} \diamond \cdots \diamond \mathcal{I}_{n}$.

Then there is a sequence of morphisms $X=S_{0} \stackrel{f_{1}}{\longrightarrow} S_{1} \longrightarrow \cdots \stackrel{f_{n}}{\longrightarrow} S_{n}$ in $\mathcal{S}$ such that

1. $\operatorname{Hom}\left(-, S_{i-1}\right) \stackrel{f_{i *}}{\longrightarrow} \operatorname{Hom}\left(-, S_{i}\right)$ vanishes on $\mathcal{I}_{i}$ and

2. $f_{1} \cdots f_{n} \neq 0$. 
Proof. First we want to construct, for any object $S \in \mathcal{S}$ and any skeletally small $\mathcal{I} \subset \mathcal{T}$ a universal morphism Add $I \longrightarrow S$. For that we take

$$
\alpha_{\mathcal{I}, S}: \coprod_{I \in \mathcal{I} / \cong \varphi \in \operatorname{Hom}(I, S)} I \longrightarrow S
$$

where the morphism in component $(I, \varphi)$ is $\varphi$. Here $I \in \mathcal{I} / \cong$ means that the coproduct runs over the isomorphism classes of objects in $\mathcal{I}$. Denote the cone of $\alpha_{\mathcal{I}, S}$ by $S \stackrel{\beta_{\mathcal{I}, S}}{\longrightarrow} C_{\mathcal{I}, S}$. Since any morphism from an element of $\mathcal{I}$ to $S$ factors through $\alpha_{\mathcal{I}, S}$, its composition with $\beta_{\mathcal{I}, S}$ is zero.

Now we define $f_{i}$ and $S_{i}$ inductively by

- $S_{0}=X$,

- $f_{i}=\beta_{\mathcal{I}_{i}, S_{i-1}}$ and

- $S_{i}=C_{\mathcal{I}_{i}, S_{i-1}}$.

Then the first claim of the lemma holds by construction, so it only remains to show that $f_{1} \cdots f_{n} \neq 0$. We will show, by induction on $i$, that the composition $f_{1} \cdots f_{i}$ is the cone of a map $I_{i} \longrightarrow X$ with $I_{i} \in\left\langle\operatorname{ads} \mathcal{I}_{1}\right\rangle \diamond \cdots \diamond\left\langle\operatorname{ads} \mathcal{I}_{i}\right\rangle$. For $i=1$ this holds by construction. So let $i>1$. Then we have the following diagram, which we may complete with the dashed arrows by the octahedral axiom.

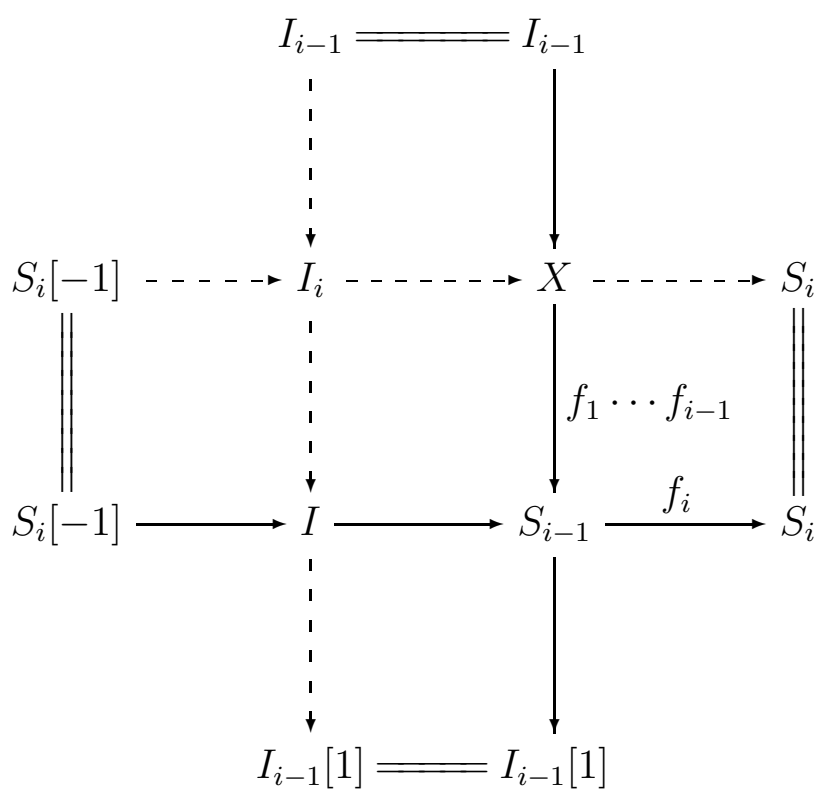

Here $I \in\left\langle\operatorname{ads} \mathcal{I}_{i}\right\rangle$ by construction, and $I_{i-1} \in\left\langle\operatorname{ads} \mathcal{I}_{1}\right\rangle \diamond \cdots \diamond\left\langle\operatorname{ads} \mathcal{I}_{i-1}\right\rangle$ inductively, so $I_{i} \in\left\langle\operatorname{ads} \mathcal{I}_{1}\right\rangle \diamond \cdots \diamond\left\langle\operatorname{ads} \mathcal{I}_{i}\right\rangle$ as claimed. 
Now assume the $f_{1} \cdots f_{n}=0$. Then $I_{n} \longrightarrow X$ is a split epimorphism. Therefore $X \in\left\langle\operatorname{ads} \mathcal{I}_{1}\right\rangle \diamond \cdots \diamond\left\langle\operatorname{ads} \mathcal{I}_{i}\right\rangle$, and by Corollary 2.2.15, $X \in\left\langle\mathcal{I}_{1}\right\rangle \diamond$ $\cdots \diamond\left\langle\mathcal{I}_{i}\right\rangle$ contradicting the assumption.

2.2.17 Remark. Note that, for $\mathcal{T}=\Lambda$ - $\underline{\bmod }$ or $\mathcal{T}=D^{b}\left(\Lambda\right.$-mod) " $\mathcal{I}_{i}$ skeletally small" is no restriction.

\subsection{Geometry}

Throughout this section we assume $X$ to be a reduced noetherian scheme over $k$. We denote by $\operatorname{coh} X$ the category of coherent sheaves on $X$.

2.3.1 Lemma. Let $\mathcal{F} \in \operatorname{coh} X$. Then there is an open dense subset $U \subseteq X$ such that the stalk $\mathcal{F}_{x}$ is a free $\mathcal{O}_{x}$-module for every $x \in U$.

Proof. If $X$ is not irreducible we treat each irreducible component of $X$ separately. Therefore we may assume that $X$ is irreducible, and hence integral. Further, by substituting $X$ by a dense open subscheme if necessary, we may assume $X$ to be affine, say $X=\operatorname{Spec} R$. Then $R$ is an integral domain, and the local ring at the generic point $\{0\}$ is the quotient field of $R$. Hence $\mathcal{F}_{\{0\}}$ is free. Now note that the set containing only the generic point is dense.

The set $\mathcal{U}=\left\{x \in X \mid \mathcal{F}_{x}\right.$ free $\}$ is open by [13, Theorem 4.10(ii)].

2.3.2 Proposition. Let $X$ be a reduced noetherian scheme over a field. Then

$$
\operatorname{dim} D^{b}(\operatorname{coh} X) \geq \operatorname{dim} X .
$$

Proof. Set $d=\operatorname{dim} X$. Assume $\mathcal{M} \in D^{b}(\operatorname{coh} X)$. We have to show that there is $\mathcal{P} \in D^{b}(\operatorname{coh} X)$ such that $\mathcal{P} \notin\langle\mathcal{M}\rangle_{n}$.

Set $\mathcal{N}=\oplus H^{i} \mathcal{M} \in \operatorname{coh} X$. Choose an irreducible component $Y$ of $X$ such that $\operatorname{dim} Y=n$. By Lemma 2.3.1 the set $\left\{p \in Y \mid \mathcal{N}_{p}\right.$ is projective $\}$ is open and dense in $Y$. Therefore it contains a point $p$ which is closed and regular in $X$. Let $\mathcal{P}$ be the sheaf which is the quotient field $k_{p}$ of the local ring $\mathcal{O}_{X}(p)$ in $p$ and vanishes everywhere else. Assume $\mathcal{P} \in\langle\mathcal{M}\rangle_{n}$. Then $\mathcal{P}_{p} \in\left\langle\mathcal{M}_{p}\right\rangle_{n}$, since localization is exact. But since $\mathcal{N}_{p}$ is projective, $\left\langle\mathcal{M}_{p}\right\rangle=\left\langle\mathcal{O}_{X}(p)\right\rangle$. Therefore $k_{p} \in\left\langle\mathcal{O}_{X}(p)\right\rangle_{n}$, so by Corollary 2.2 .11 we have $\operatorname{pd} k_{p}<n$. This contradict our assumption on the dimension of $Y$.

\subsection{Known examples of algebras with representation dimension larger than three}

The explicit examples here (2.4.1 and 2.4.3) will be generalized (and in the second case improved) in Section 7 . We include a sketch of the original proofs 
here anyway, since they are not very long from what we already know from the previous subsections.

2.4.1 Theorem (Rouquier [16]). Let $\Lambda_{n}(0 \neq n \in \mathbb{N})$ be the exterior algebra on an $n$-dimensional vector space. Then

$$
\operatorname{repdim} \Lambda_{n}=n+1 \text {. }
$$

Proof. By Koszul duality, there is an equivalence between the bounded derived category of $\Lambda_{n}$-mod and the derived category of differential graded modules over the polynomial ring $k\left[x_{1}, \ldots, x_{n}\right]$. This equivalence takes $\Lambda_{n}$ to $k$, and therefore maps $\Lambda_{n}$-perf to the smallest triangulated subcategory which contains $k$. In particular, it also induces an equivalence between the quotients. Now note that $k\left[x_{1}, \cdots, x_{n}\right]-\bmod _{\mathrm{gr}} /(\operatorname{modules}$ of finite length $) \approx$ $\operatorname{coh} \mathbb{P}^{n-1}$ and apply Proposition 2.3.2 and 2.2.7.

2.4.2 Theorem (Krause, Kussin [12]). Let $X$ be a reduced projective scheme over $k, \mathcal{F} \in \operatorname{coh} X$ a tilting sheaf. Then

$$
\operatorname{repdim} \operatorname{End}(\mathcal{F})^{\text {op }} \geq \operatorname{dim} X
$$

Proof. There is an equivalence $D^{b}(\operatorname{coh} X) \approx D^{b}\left(\operatorname{End}(\mathcal{F})^{\text {op }}\right.$-mod $)$. Therefore $\operatorname{dim} D^{b}\left(\operatorname{End}(\mathcal{F})^{\text {op }}\right.$-mod $) \geq \operatorname{dim} X$ by Proposition 2.3.2. Now the claim follows from Proposition 2.2.7.

2.4.3 Theorem (Krause, Kussin [12]). Let $\mathrm{k}$ be a field, $\Lambda_{N}=k Q / I$ with

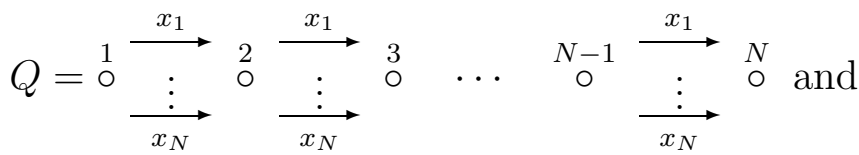

$$
\begin{aligned}
& I=\left(x_{n} x_{m}-x_{m} x_{n}\right) \text {. }
\end{aligned}
$$

Then repdim $\Lambda_{N} \geq N-1$.

Proof. The algebra of this theorem arises in the way described in Theorem 2.4.2 for $X=\mathbb{P}^{N-1}$ and $\mathcal{F}=\oplus_{i=0}^{N-1} \mathcal{O}_{X}(i)$. 


\section{Group algebras}

In this section we try to construct lower bounds for the representation dimension of group algebras. The first and main subsection is devoted to group algebras of elementary abelian groups. We show that their representation dimension is bounded below by the rank of the elementary abelian group plus one (Theorem 1). It is not known whether equality holds, but it should be noted that the group is representation finite if and only if the rank is at most one.

In the second subsection we carry over the results of Subsection 3.1 to arbitrary blocks of group algebras. This leads to lower bounds for their representation dimension (Corollary 3.2.4).

\subsection{Elementary abelian groups}

Let $p$ be a prime. The elementary abelian group of rank $n$ is $\left(C_{p}\right)^{n}$, where $C_{p}$ denotes the cyclic group of order $p$. We assume $k$ to be a field of characteristic $p$ (for other fields the group algebra $k C_{p}^{n}$ is semisimple, hence the representation dimension is 0 ). The aim of this section is to prove the following theorem.

Theorem 1. Let $k$ be a field of characteristic $p$. Then

$$
\operatorname{dim} k C_{p}^{n}-\underline{\bmod } \geq n-1 .
$$

In particular

$$
\text { repdim } k C_{p}^{n} \geq n+1 \text {. }
$$

For simplicity of notation we set $\Lambda=k C_{p}^{n}$.

Before we start with the proof of the theorem, note that

$$
\Lambda \cong S(V) /\left(v^{p} \mid v \in V\right)
$$

where $V$ is an $n$-dimensional $k$-vector space, $S(V)$ is the symmetric algebra and $\left(v^{p} \mid v \in V\right)$ the ideal generated by all $p$-th powers of elements of $V$. In this description of $\Lambda$, all non-zero $v \in V$ play the same role. This symmetry will help us in the proof.

\section{Proof of Theorem 1 in the case $k$ is infinite}

In this subsection we assume the field $k$ to be infinite. Whenever we are talking about open or closed sets we are referring to the classical Zariski topology. 
3.1.1 Lemma. Let $\mathcal{Y} \subset \bmod -\Lambda$ be a finite set of right $\Lambda$-modules. Then there is an open, nonempty subset $\mathcal{U} \subset V$, such that for any $u \in \mathcal{U}$, any $y \in Y \in \mathcal{Y}$, and any $1 \leq s<p$ we have

$$
y u=0 \Rightarrow y \cdot \operatorname{Rad}^{s} \Lambda \subset Y u^{s} .
$$

Proof. Fix $1 \leq s<p$ and $Y \in \mathcal{Y}$. Any $v \in V$ induces a linear map

$$
\rho_{v}^{s}: Y \longrightarrow Y: y \longmapsto y v^{s} .
$$

Now we can find a set with the desired property for our fixed $s$ and $Y$ :

$$
\mathcal{U}_{s, Y}=\left\{v \in V \mid \operatorname{rk} \rho_{v}^{s} \text { maximal }\right\} .
$$

By choosing a basis for $V$ and $Y$, the maps $\rho_{v}^{s}$ induce a polynomial map

$$
k^{\operatorname{dim} V} \cong V \longrightarrow \operatorname{End}_{k} Y \cong k^{\operatorname{dim} Y \times \operatorname{dim} Y} .
$$

We then compose this map with taking subdeterminants of size $r$, where $r$ is the maximal rank in the definition of $\mathcal{U}_{s, Y}$ above. This results in polynomial maps $k^{\operatorname{dim} V} \longrightarrow k$, such that $\mathcal{U}_{s, Y}$ is just the set where not all of these polynomials are zero. Thus $\mathcal{U}_{s, Y}$ is open and obviously it is non-empty.

Now fix $u \in \mathcal{U}_{s, Y}$. Let $\left\{a_{i} \mid 1 \leq i \leq A\right\}$ be a basis of $\operatorname{Ker} \rho_{u}^{1}$, complement it to a basis $\left\{a_{i}, b_{j} \mid 1 \leq i \leq A, 1 \leq j \leq B\right\}$ of $\operatorname{Ker} \rho_{u}^{s}$, and finally to a basis $\left\{a_{i}, b_{j}, c_{l} \mid 1 \leq i \leq A, 1 \leq j \leq B, 1 \leq l \leq C\right\}$ of $Y$.

Let $v \in V$. The rank of $\rho_{u}^{s}$ is maximal, so in particular $\operatorname{rk} \rho_{u+\varepsilon v}^{s} \leq \operatorname{rk} \rho_{u}^{s}$ for any $\varepsilon$. Fix $1 \leq i \leq A$. Therefore, for all $\varepsilon$, the tuple

$$
\left(a_{i} \rho_{u+\varepsilon v}^{s}, c_{1} \rho_{u+\varepsilon v}^{s}, \ldots, c_{C} \rho_{u+\varepsilon v}^{s}\right)
$$

is linearly dependent. Since $a_{i} u=0$, for $\varepsilon \neq 0$ the tuple

$$
\left(a_{i} \rho_{v}^{s}, c_{1} \rho_{u+\varepsilon v}^{s}, \ldots, c_{C} \rho_{u+\varepsilon v}^{s}\right)
$$

also is linearly dependent. But the set of all $\varepsilon$ such that it is linearly dependent is closed (the subdeterminants of $\left(a_{i} \rho_{v}^{s}, c_{l} \rho_{u+\varepsilon v}^{s}\right)$ are polynomials in $\varepsilon$ ), hence it has to be all of $k$. Therefore, especially $\left(a_{i} \rho_{v}^{s}, c_{l} \rho_{u}^{s} \mid 1 \leq l \leq C\right)$ is linearly dependent, so $a_{i} \rho_{v}^{s} \in\left\langle c_{l} \rho_{u}^{s} \mid 1 \leq l \leq C\right\rangle$. But the $v^{s}$ generate $\operatorname{Rad}^{s} \Lambda$ as a $k$-vector space, since $s$ is strictly smaller than $p$.

Finally set $\mathcal{U}=\bigcap_{Y \in \mathcal{Y}} \bigcap_{s=1}^{p-1} \mathcal{U}_{s, Y}$.

3.1.2 Lemma. Let $\mathcal{X} \subset \Lambda$-mod be a finite set of $\Lambda$-modules. Then there is an open, nonempty subset $\mathcal{U} \subset V$, such that for any $u \in \mathcal{U}$, any $\varphi \in$ $\operatorname{Hom}_{\Lambda}(X, \Lambda)$ with $X \in \mathcal{X}$, and any $1 \leq s<p$ we have

$$
\varphi u=0 \Rightarrow \varphi \cdot \operatorname{Rad}^{s} \Lambda \subset \operatorname{Hom}_{\Lambda}(X, \Lambda) u^{s} .
$$


Proof. Set $\mathcal{Y}=\left\{\operatorname{Hom}_{\Lambda}(X, \Lambda) \mid X \in \mathcal{X}\right\}$ in Lemma 3.1.1.

3.1.3 Proposition. Let $\mathcal{X} \subset \Lambda$-mod be a finite set of $\Lambda$-modules. Let $u \in \mathcal{U}$ of Lemma 3.1.2, $N=\Lambda u^{p-1}$. Then, for any $X \in \mathcal{X}$ and any $f \in \operatorname{Rad}^{p-1} \Lambda$, any composition $X \longrightarrow N \stackrel{\cdot f}{\longrightarrow} N$ factors through $\Lambda$-proj.

Proof. Fix $X \in \mathcal{X}$. Since $N$ is a submodule of $\Lambda$, we may identify

$$
\begin{aligned}
\operatorname{Hom}_{\Lambda}(X, N) & =\left\{\varphi \in \operatorname{Hom}_{\Lambda}(X, \Lambda) \mid \varphi(X) \subset N\right\} \\
& =\left\{\varphi \in \operatorname{Hom}_{\Lambda}(X, \Lambda) \mid \varphi(X) \subset \Lambda u^{p-1}\right\} \\
& =\left\{\varphi \in \operatorname{Hom}_{\Lambda}(X, \Lambda) \mid \varphi(X) u=0\right\}
\end{aligned}
$$

Let $\varphi \in \operatorname{Hom}_{\Lambda}(X, N)$, that is $\varphi \in \operatorname{Hom}_{\Lambda}(X, \Lambda)$ with $\varphi u=0$. Then, by Lemma 3.1.2, $\varphi f \in \operatorname{Hom}_{\Lambda}(X, \Lambda) u^{p-1}$.

The projective cover of $N$ is induced by the endomorphism $u^{p-1}$ of $\Lambda$. Therefore, the maps $X \longrightarrow N$ factoring through a projective module are exactly the elements of $\operatorname{Hom}_{\Lambda}(X, \Lambda) u^{p-1}$.

3.1.4 Proposition. Let $v \in V \backslash\{0\}, N=\Lambda v^{p-1}$. Then the composition

$$
N \longrightarrow \text { hd } N \cong k \cong \operatorname{Soc} N \longrightarrow N
$$

does not factor through $\Lambda$-proj.

Proof. Let $\left\{v_{1}, v_{2}, \ldots, v_{n}\right\}$ be a basis of $V, v_{1}=v$. Then the composition above is (up to a scalar) multiplication with $\prod_{i \neq 1} v_{i}^{p-1}$. Let $f$ be any map $\Lambda \longrightarrow N$. It is defined by the image of $1_{\Lambda}$ in $N \subset \Lambda$, which we will also call $f$. Now assume that the following diagram commutes.

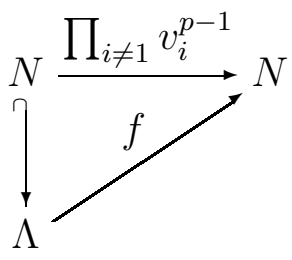

The image of $v^{p-1}$ has to be the same on both ways, that is $v^{p-1} f=\prod_{i} v_{i}^{p-1}$. Therefore $f-\prod_{i \neq 1} v_{i}^{p-1}$ is a multiple of $v$. Since $f \in N, f$ is also a multiple of $v$. So $\prod_{i \neq 1} v_{i}^{p-1}$ would be a multiple of $v$, but that is not true in $\Lambda$.

Therefore the morphism cannot factor through $\Lambda$-proj.

Proof of Theorem 1 for infinite fields. Let $M$ be a $\Lambda$-module realizing the minimal $d$ in the definition of the dimension of the stable module category. Let $\mathcal{X}=\{M, \mho M\}$, where $\mho$ is the cosyzygy functor, which is the shift in 
the stable category. Then choose $u \in \mathcal{U}$ as in Lemma 3.1.2, and complement it to a basis $\left\{u_{1}, \ldots u_{n}\right\}$ of $\mathrm{V}$ with $u_{1}=u$. Let $N=\Lambda u^{p-1}$. Then we have the following sequence of cohomological functors $\Lambda$-mod $\longrightarrow k$-mod.

$$
\underline{\operatorname{Hom}}_{\Lambda}(-, N) \stackrel{\cdot u_{2}^{p-1}}{\longrightarrow} \underline{\operatorname{Hom}}_{\Lambda}(-, N) \cdots \stackrel{\cdot u_{n}^{p-1}}{\longrightarrow} \underline{\operatorname{Hom}}_{\Lambda}(-, N)
$$

Its composition is nonzero by Proposition 3.1.4. So by Corollary 2.2.10 we only need to show that $\underline{\operatorname{Hom}}_{\Lambda}\left(\mho^{i} M, N\right) \stackrel{\cdot u_{j}^{p-1}}{\longrightarrow} \underline{\operatorname{Hom}}_{\Lambda}\left(\mho^{i} M, N\right)$ is zero for any $i \in \mathbb{Z}$ and any $2 \leq j \leq n$.

The following diagrams have short exact rows and commute for any $f \in \Lambda$.
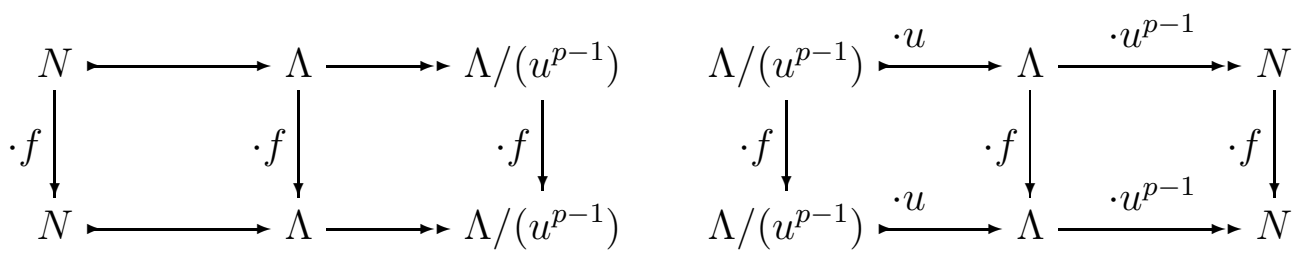

Therefore $\mho N N \cong \Lambda /\left(u^{p-1}\right), \mho\left(\Lambda /\left(u^{p-1}\right)\right) \cong N$ and $\mho(\stackrel{\cdot f}{\longrightarrow}) \cong \stackrel{\cdot f}{\longrightarrow}$. So there is a commutative diagram

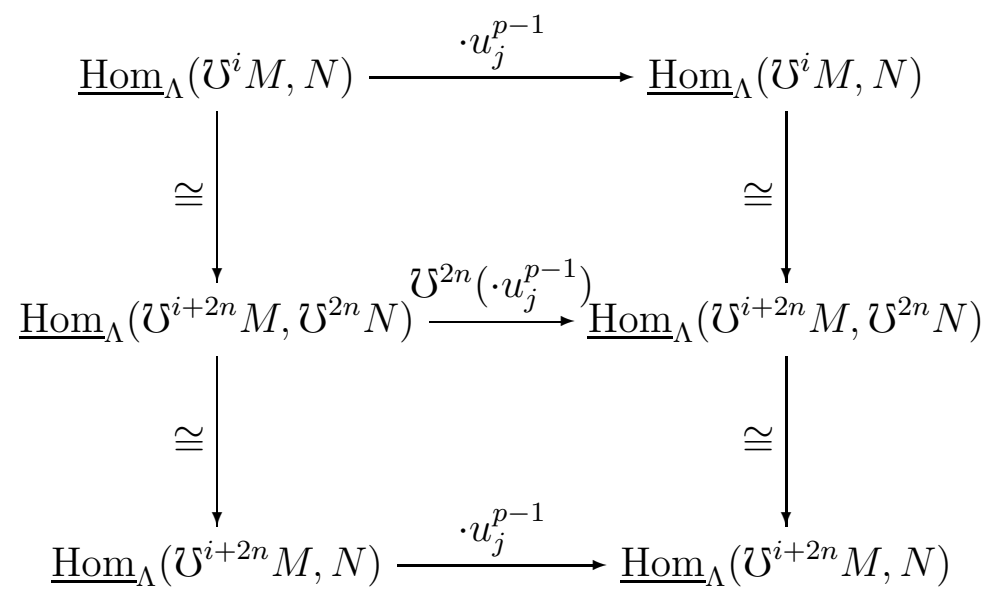

By choosing $n$ appropriately we can get $i+2 n \in\{0,1\}$, so $\mho^{i+2 n} M \in \mathcal{X}$ and the claim follows from Proposition 3.1.3.

\section{The case of a finite field}

Now let $k$ be finite, $\bar{k}$ an algebraic closure. Denote by $\bar{\Lambda}=\bar{k} \otimes_{k} \Lambda$ the induced algebra. For any $\Lambda$-module $X$ let $\bar{X}=\bar{k} \otimes_{k} X \in \bar{\Lambda}$-mod, and for $\mathcal{X} \subset \Lambda$-mod let $\overline{\mathcal{X}}=\{\bar{X} \mid X \in \mathcal{X}\}$. Whenever we are talking about an extension field $\widehat{k}$ of $k$ let $\widehat{\Lambda}, \widehat{X}$ and $\widehat{\mathcal{X}}$ be the obvious variations of the above. 
3.1.5 Lemma. Let $\mathcal{X} \subset \Lambda$-mod be finite. Then there is a finite extension $\widehat{k}$ of $k$ and $u \in \widehat{V}$ such that any $\varphi \in \operatorname{Hom}_{\widehat{\Lambda}}(\widehat{X}, \widehat{\Lambda})$ with $X \in \mathcal{X}$, and any $1 \leq s<p$ we have

$$
\varphi u=0 \Rightarrow \varphi \cdot \operatorname{Rad}^{s} \widehat{\Lambda} \subset \operatorname{Hom}_{\widehat{\Lambda}}(\widehat{X}, \widehat{\Lambda}) u^{p-1}
$$

Proof. By Lemma 3.1.2 there is $u \in \bar{V}$ such that for any $\varphi \in \operatorname{Hom}_{\bar{\Lambda}}(\bar{X}, \bar{\Lambda})$ and any $1 \leq s<p$ we have $\varphi u=0 \Rightarrow \varphi \cdot \operatorname{Rad}^{s} \bar{\Lambda} \subset \operatorname{Hom}_{\bar{\Lambda}}(\bar{X}, \bar{\Lambda}) u^{s}$. Choose $\widehat{k}$ finite over $k$ such that $u \in \widehat{V}$. Since $\bar{k} \otimes_{\widehat{k}} \operatorname{Hom}_{\widehat{\Lambda}}(\widehat{X}, \widehat{\Lambda})=\operatorname{Hom}_{\bar{\Lambda}}(\bar{X}, \bar{\Lambda})([7,29.5])$, we may identify $\operatorname{Hom}_{\widehat{\Lambda}}(\widehat{X}, \widehat{\Lambda})$ with the subset of morphisms in $\operatorname{Hom}_{\bar{\Lambda}}(\bar{X}, \bar{\Lambda})$ mapping $\widehat{X}$ to $\widehat{\Lambda}$. Therefore, for any $\varphi \in \operatorname{Hom}_{\widehat{\Lambda}}(\widehat{X}, \widehat{\Lambda})$ with $\varphi u=0$, we have $\varphi \cdot \operatorname{Rad}^{s} \widehat{\Lambda} \subset \operatorname{Hom}_{\bar{\Lambda}}(\bar{X}, \bar{\Lambda}) u^{s} \cap \operatorname{Hom}_{\widehat{\Lambda}}(\widehat{X}, \widehat{\Lambda})=\operatorname{Hom}_{\widehat{\Lambda}}(\widehat{X}, \widehat{\Lambda}) u^{s}$. The right equality holds because $\widehat{\Lambda}$ is a direct summand of $\bar{\Lambda}$ as $\widehat{\Lambda}$-module.

3.1.6 Proposition. Let $\mathcal{X} \subset \Lambda-\bmod$ be finite, $u$ and $\widehat{k}$ as in Lemma 3.1.5, and set $N=\widehat{\Lambda} u^{p-1}$. Then, for any $X \in \mathcal{X}$ and any $f \in \operatorname{Rad}^{p-1} \widehat{\Lambda}$, any composition $\widehat{X} \longrightarrow N \stackrel{\cdot f}{\longrightarrow} N$ of $\widehat{\Lambda}$-morphisms factors through $\widehat{\Lambda}$-proj.

Proof. This is just the proof of Proposition 3.1.3, replacing the reference to Lemma 3.1.2 by a reference to Lemma 3.1.5.

3.1.7 Proposition. Let $\mathcal{X} \subset \Lambda-\bmod$ finite, $u$ and $\widehat{k}$ as in Lemma 3.1.5, and set $N=\widehat{\Lambda} u^{p-1}$. Then, for any $X \in \mathcal{X}$ and any $f \in \operatorname{Rad}^{p-1} \widehat{\Lambda}$, any composition $X \longrightarrow N \stackrel{\cdot f}{\longrightarrow} N$ of $\Lambda$-morphisms factors through $\Lambda$-proj.

Proof. Any $\Lambda$-morphism $\varphi: X \longrightarrow N$ lifts to a $\widehat{\Lambda}$-morphism $\widehat{\varphi}: \widehat{X} \longrightarrow N$ as indicated in the following diagram.

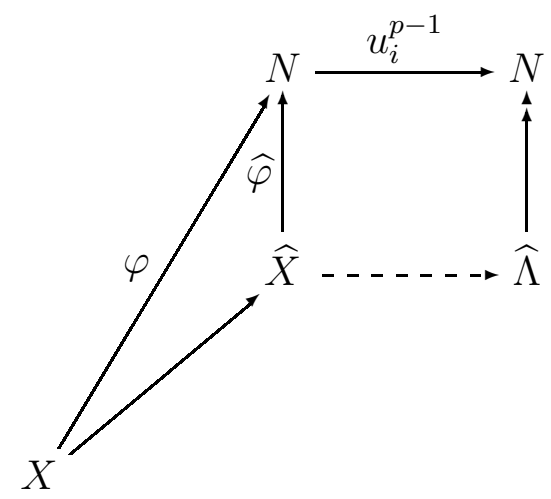

The dashed arrow now exists by Proposition 3.1.6 making the square commutative, so the composition factors through $\widehat{\Lambda}$. Clearly this is a projective $\Lambda$-module. 
We note that Proposition 3.1.4 does not depend on the field at all. To see that the morphism is non-zero in $\Lambda-\underline{\bmod }$, not just in $\widehat{\Lambda}-\underline{\bmod }$, we need to recall the following lemma.

3.1.8 Lemma. Let $\Lambda \subset \widehat{\Lambda}$ be finite dimensional algebras. Assume $\widehat{\Lambda}$ is projective as $\Lambda$-module and a direct summand of $\widehat{\Lambda} \otimes_{\Lambda} \widehat{\Lambda}$ as $(\widehat{\Lambda}, \widehat{\Lambda})$-bimodule. Then restriction induces an injective map $\underline{\operatorname{Hom}}_{\widehat{\Lambda}}(X, Y) \longrightarrow \underline{\operatorname{Hom}}_{\Lambda}(X, Y)$.

Proof. Assume a $\widehat{\Lambda}$-morphism $\varphi: X \longrightarrow Y$ vanishes in $\underline{\operatorname{Hom}}_{\Lambda}(X, Y)$. Then it factors through a finite number of copies of $\Lambda$, as indicated in the following diagram.

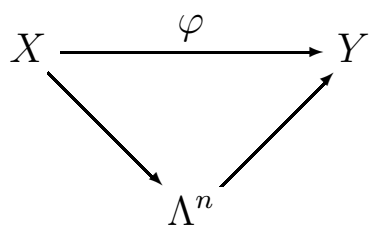

Let $\widehat{\Lambda} \underset{\pi}{\stackrel{\iota}{\rightleftarrows}} \widehat{\Lambda} \otimes_{\Lambda} \widehat{\Lambda}$ be the maps inducing the direct sum decomposition of $\widehat{\Lambda} \otimes_{\Lambda} \widehat{\Lambda}$. Tensoring the above diagram with $\widehat{\Lambda}$ we find the triangle in the following diagram. The rest of the diagram commutes since tensoring commutes with direct sums.

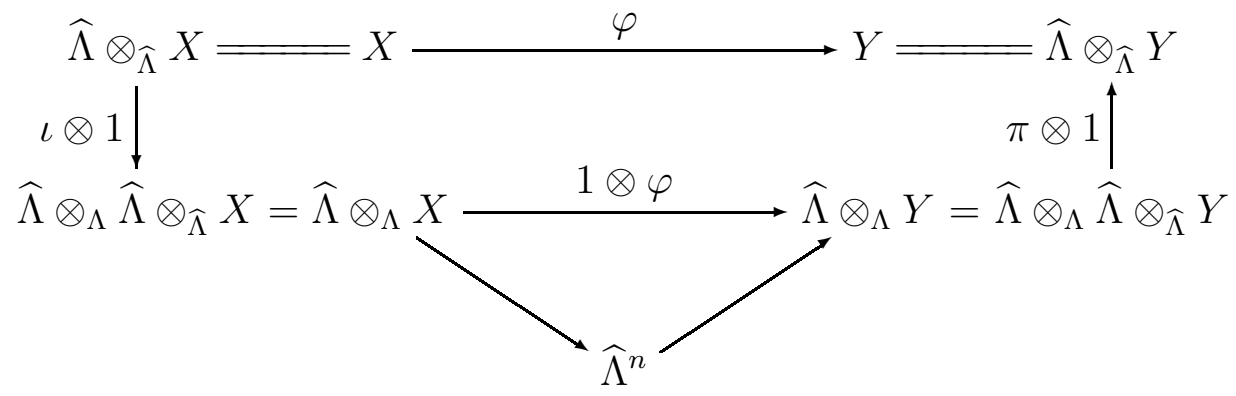

Therefore $\varphi$ also vanishes in $\underline{\operatorname{Hom}}_{\widehat{\Lambda}}(X, Y)$.

3.1.9 Corollary. Let $\widehat{k}$ be a finite extension field of $k, v \in \widehat{V}$. Then the composition $\psi: \widehat{\Lambda} v^{p-1} \longrightarrow \widehat{k} \longrightarrow \widehat{\Lambda} v^{p-1}$ does not factor through a projective $\Lambda$-module.

Proof. Since $k$ is a finite field the extension is separable. Therefore, by [7, Corollary 69.8] $\widehat{k}$ is a direct summand of $\widehat{k} \otimes_{k} \widehat{k}$ as $(\widehat{k}, \widehat{k})$-bimodule. Tensoring with $\Lambda$ we find that the assumptions of Lemma 3.1 .8 are satisfied.

Proof of Theorem 1 for finite fields. The argument is the same as the one in the proof of Theorem 1 for infinite fields at the end of Section 3, with 
references to Propositions 3.1.3 and 3.1.4 replaced by references to Proposition 3.1.7 and Corollary 3.1.9 respectively. However, when we choose $M$ as before and set $\mathcal{X}=\{M, \mho M\}$, we need to check that $\widehat{\mathcal{X}}$ is indeed $\left\{\widehat{M}, \mho_{\widehat{\Lambda}} \widehat{M}\right\}$, or, in other words, that taking cosyzygies and tensoring with $\widehat{k}$ commutes. This is the case because tensoring with $\widehat{k}$ is exact and $\widehat{k} \otimes \Lambda$ is projective over itself.

\subsection{Finite groups in general - Benson's Conjecture}

The applications here are obtained by applying the ideas of Rouquier (in [16]) to the more general result.

3.2.1 Proposition (implicit in [16, Theorem 4.9]). Let $H \leq G$ be finite groups. Then

$$
\operatorname{dim} k G-\underline{\bmod } \geq \operatorname{dim} k H-\underline{\bmod } .
$$

Proof. We have the exact functors

$$
\begin{aligned}
& \text { res }: k G-\bmod \longrightarrow k H-\bmod \text { and } \\
& \text { ind }: k H-\bmod \longrightarrow k G-\bmod .
\end{aligned}
$$

Since both of them map projective modules to projective ones there are induced triangle functors $k G-\underline{\bmod } \rightleftarrows k H-\underline{\bmod }$. By Lemma 2.2.2, it suffices to show that every $k H$-module is a direct summand of a module in the image of res. But $k H$ is a direct summand of $k G$ as $k H$ - $k H$-bimodule, so $1_{k H \text {-mod }}$ is a direct summand of res o ind.

3.2.2 Corollary. Let $G$ be a finite group, $k$ a field of characteristic $p$, such that $p$ divides the order of $G$. Then

$$
\mathrm{LL} k G \geq \operatorname{repdim} k G \geq \operatorname{dim} k G-\underline{\bmod }+2>p-\operatorname{rank}(G) .
$$

Proof. The first inequality is Lemma 2.1.8, the second one is Lemma 2.2.5. The third inequality follows from Theorem 1 and Proposition 3.2.1.

3.2.3 Proposition ([16, Proposition 4.7]). Let $G$ be a finite group and $B$ a block of $k G$. Let $D$ be a defect group of $B$. Then $\operatorname{dim} B-\underline{\bmod }=$ $\operatorname{dim} k D$ - $\underline{\bmod }$.

The proof is similar to the one of Proposition 3.2.1. See [16].

3.2.4 Corollary. Let $G$ be a finite group, $B$ a non-semisimple block of $k G$, char $k=p$. Let $D$ be a defect group of $B$. Then

$$
\mathrm{LL}(B) \geq \operatorname{repdim} B \geq \operatorname{dim} B-\underline{\bmod }+2=\operatorname{dim} D-\underline{\bmod }+2>p-\operatorname{rank}(D) .
$$


In particular, we have obtained the following inequality, which had been conjectured by Benson without connection to representation dimension.

3.2.5 Corollary (Benson's conjecture). Let $G$ be a finite group, $B$ a block of $k G$, char $k=p$. Let $D$ be a defect group of $B$. Then

$$
\mathrm{LL}(B)>p-\operatorname{rank}(D) .
$$




\section{The dimension of a module category}

Now we generalize Rouquier's definition of dimension of a triangulated category to subcategories of triangulated categories. Since the module category is a subcategory of its derived category (by identifying it with the complexes concentrated in degree 0) we obtain a notion of dimension of a module category. We show that this dimension can also be used to establish lower bounds for the representation dimension (Lemmas 4.4 and 4.5), and give some indication that it gives rise to good lower bounds (Corollaries 4.11 and 4.15).

Let $\mathcal{T}$ be a triangulated category, $\mathcal{I}$ a subcategory. We use $*, \diamond$ and $\langle-\rangle_{n}$ as in 2.2 .

4.1 Definition. Let $\mathcal{T}$ be a triangulated category, $\mathcal{C} \subseteq \mathcal{T}$. Let $M \in$ ob $\mathcal{T}$. We define the $M$-level of $\mathcal{C}$ to be

$$
M-\text { level }_{\mathcal{T}} \mathcal{C}=\min \left\{n \in \mathbb{N} \mid \mathcal{C} \subseteq\langle M\rangle_{n+1}\right\},
$$

and the dimension of $\mathcal{C}$ to be

$$
\operatorname{dim}_{\mathcal{T}} \mathcal{C}=\min _{M \in O G \mathcal{T}} M-\operatorname{level}_{\mathcal{T}} \mathcal{C}
$$

Note that for $\mathcal{C}=\mathcal{T}$ this coincides with Rouquier's definition (2.2.1, $[15,16])$ of dimension of a triangulated category.

We will omit the index $\mathcal{T}$ whenever there is no danger of confusion. Especially, whenever $\mathcal{C} \subseteq \Lambda$-mod for some finite dimensional algebra $\Lambda$, we assume the triangulated category to be $D^{b}(\Lambda$-mod) unless otherwise specified.

The following lemma is an immediate consequence of the definition, and part 2 is also an analog of Lemma 2.2.2.

4.2 Lemma. 1. Assume $\mathcal{C} \subseteq \mathcal{D} \subseteq \mathcal{T}$ for a triangulated category $\mathcal{T}$. Then $\operatorname{dim} \mathcal{C} \leq \operatorname{dim} \mathcal{D}$.

2. Let $\mathrm{F}: \mathcal{T} \longrightarrow \mathcal{T}^{\prime}$ be a triangulated functor. Let $\mathcal{C} \subseteq \mathcal{T}$. Then $\operatorname{dim}_{\mathcal{T}} F(\mathcal{C}) \leq \operatorname{dim}_{\mathcal{T}} \mathcal{C}$

To (hopefully) simplify notation, we introduce the following two resolution dimensions.

4.3 Definition. Let $M$ be a finite dimensional $\Lambda$-module, $X \in \Lambda$-mod and $\mathcal{X} \subseteq \Lambda$-mod. 
1. The weak resolution dimension of the module $X$ and the class of modules $\mathcal{X}$ are defined by

$$
\begin{aligned}
M \text {-wresol.dim } X= & \inf \{n \mid \exists \text { an exact sequence } \\
& M_{n} \longrightarrow M_{n-1} \longrightarrow \cdots \longrightarrow M_{0} \longrightarrow X \\
& \text { with } \left.M_{i} \in \text { add } M\right\}, \\
M \text {-wresol.dim } \mathcal{X}= & \max \{M \text {-wresol.dim } X \mid X \in \mathcal{X}\},
\end{aligned}
$$

and

$$
\text { wresol.dim } \mathcal{X}=\inf \{M \text {-wresol.dim } \mathcal{X} \mid M \in \Lambda \text {-mod }\} \text {. }
$$

2. The (universal) resolution dimension of the module $X$ and the class of modules $\mathcal{X}$ are defined by

$$
\begin{gathered}
M \text {-resol.dim } X=\inf \{n \mid \exists \text { an exact sequence } \\
M_{n} \longrightarrow M_{n-1} \longrightarrow \cdots \longrightarrow M_{0} \longrightarrow X
\end{gathered}
$$

with $M_{i} \in$ add $M$, such that the induced sequence

$$
0 \longrightarrow \operatorname{Hom}_{\Lambda}\left(M, M_{n}\right) \longrightarrow \cdots \longrightarrow \operatorname{Hom}_{\Lambda}(M, X) \longrightarrow 0
$$

is also exact $\}$.

$$
M \text {-resol.dim } \mathcal{X}=\max \{M \text {-resol.dim } X \mid X \in \mathcal{X}\},
$$

and

$$
\text { resol. } \operatorname{dim} \mathcal{X}=\inf \{M \text {-resol.dim } \mathcal{X} \mid M \in \Lambda \text {-mod }\} .
$$

Finally we, to be able to compare to the representation dimension, we set

$$
\begin{aligned}
& \text { resol. } \operatorname{dim}_{\mathrm{gc}} \mathcal{X}=\inf \{M \text {-resol.dim } \mathcal{X} \mid M \in \Lambda \text {-mod } \\
& \text { generator and cogenerator }\} .
\end{aligned}
$$

(we always set $\inf \emptyset=\infty$.)

Clearly we have $M$-resol.dim $\mathcal{X} \geq M$-wresol.dim $\mathcal{X}$ and therefore also resol. $\operatorname{dim} \mathcal{X} \geq$ wresol.dim $\mathcal{X}$.

Note that the universal $M$-resolution of a module $N$ already occurred in Lemma 2.1.2. Therefore that lemma now reads as follows: 
4.4 Lemma (restatement of Lemma 2.1.2). Let $\Lambda$ be a non-semisimple finite dimensional algebra. Then

$$
\operatorname{repdim} \Lambda=\text { resol. } \operatorname{dim}_{\mathrm{gc}} \Lambda-\bmod +2 \text {. }
$$

We obtain the following connection to the dimension of a subcategory of the module category:

4.5 Lemma. Let $\Lambda$ be a finite dimensional algebra, and $M \in \Lambda$-mod. Then for any $\mathcal{X} \subseteq \Lambda-\bmod$

$$
M \text {-level } \mathcal{X} \leq M \text {-wresol.dim } \mathcal{X}
$$

and in particular

$$
\operatorname{dim} \mathcal{X} \leq \text { wresol.dim } \mathcal{X}
$$

Proof. This is an immediate consequence of the fact that short exact sequences in $\Lambda$-mod are turned into triangles in $D^{b}(\Lambda$-mod).

Let us illustrate the most important dimensions and inequalities in the following diagram, where a line means that the upper expression is larger than or equal to the lower one.

4.6. Most important inequalities:

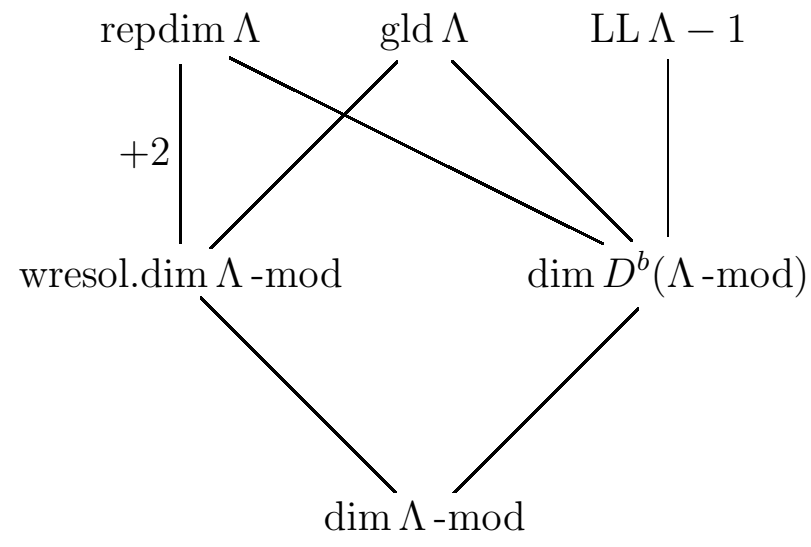

Here we will get (by two) better lower bounds for the representation dimension by using the left path in the above diagram rather than just the inequality $\operatorname{dim} D^{b}(\Lambda$-mod $) \leq \operatorname{repdim} \Lambda$.

Clearly this new path is only open, if we can find lower bounds for the dimension of a module category. We will now see how the ideas of Lemma 2.2.9 and Corollary 2.2.10 can be adjusted to that question. 
4.7 Definition. We call a map of complexes $f:\left(A_{i}, \partial_{i}^{A}\right) \longrightarrow\left(B_{i}, \partial_{i}^{B}\right)$ locally null-homotopic if for every $i$ there are maps $r_{i}$ and $s_{i}$ as indicated in the following diagram, such that $f=r_{i} \partial_{i-1}^{B}+\partial_{i}^{A} s_{i}$.

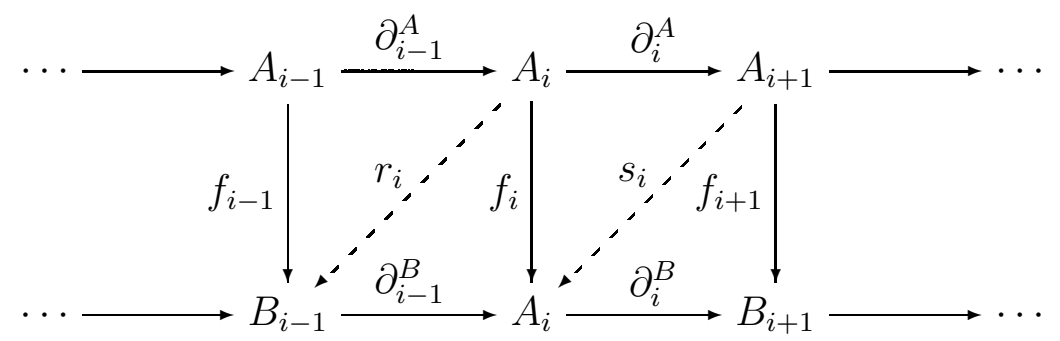

4.8 Proposition. Let $M \in D^{b}(\Lambda$-mod). Assume there is a sequence of morphisms

$$
N_{0} \stackrel{f_{1}}{\longrightarrow} N_{1} \stackrel{f_{2}}{\longrightarrow} \cdots \stackrel{f_{d}}{\longrightarrow} N_{d}
$$

in $K^{b}\left(\Lambda\right.$-inj), such that $\operatorname{Hom}_{D^{b}(\Lambda \text {-mod })}\left(M[i], N_{j-1}\right) \cdot f_{j}=0$ for all $i \in \mathbb{Z}$ and $j \in\{1, \ldots, d\}$. Assume further $f_{1} \cdots f_{d}$ is not locally null-homotopic. Then $\Lambda-\bmod \nsubseteq\langle M\rangle_{d}$.

Proof. Assume that $f_{1} \cdots f_{d}$ is not locally null-homotopic in position $i$. Let

$$
Z=\operatorname{Ker}\left[\left(N_{0}\right)_{i} \stackrel{\partial_{N_{0}}}{\longrightarrow}\left(N_{0}\right)_{i+1}\right] .
$$

Then we have a natural map $h: Z[-i] \longrightarrow N_{0}$. We will show that $h f_{1} \cdots f_{d}$ is not 0 . Then the claim follows from Lemma 2.2.9.

Assume to the contrary that $h f_{1} \cdots f_{d}=0$, that is, it is null-homotopic as a map of complexes. That means there is a map $\widetilde{r}$ as indicated in the following diagram, making the triangle commutative.

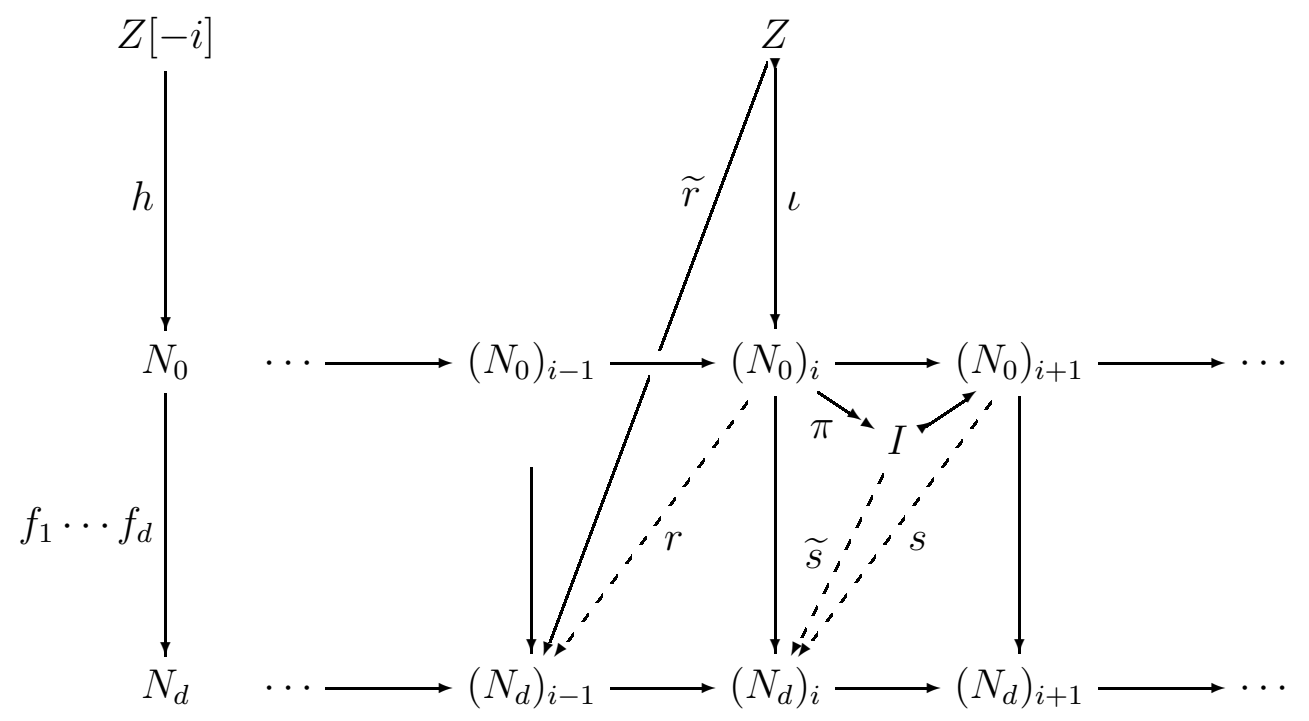


Since $\left(N_{d}\right)_{i-1}$ is injective $\widetilde{r}$ lifts to a map $r$ as indicated in the diagram. We have $0=(\widetilde{r}-\iota r) \partial_{N_{d}}=\iota\left(\left(f_{1} \cdots f_{d}\right)_{i}-r \partial_{N_{d}}\right)$, so $\left(f_{1} \cdots f_{d}\right)_{i}-r \partial_{N_{d}}$ factors through $\operatorname{cok} \iota=\pi$, say via $\widetilde{s}$. Since $\left(N_{d}\right)_{i}$ is injective $\widetilde{s}$ can be lifted to a map $s$ as indicated in the diagram. Thus $f_{1} \cdots f_{d}$ is locally null-homotopic in position $i$, contradicting the assumption.

Note that, for $\Lambda$ self-injective, Rouquier [16] also improved the lower bound he obtained for the representation dimension from $\operatorname{dim} D^{b}(\Lambda$-mod) to $\operatorname{dim} \Lambda-\underline{\bmod }+2$ by looking at the dimension of the stable module category $\Lambda$-mod rather then at the derived category. The following lemma shows that his improvement is included in ours in that case.

4.9 Lemma. Let $\Lambda$ be a self-injective finite dimensional algebra. Then

$$
\operatorname{dim} \Lambda-\bmod \geq \operatorname{dim} \Lambda-\underline{\bmod } .
$$

Proof. The functor $D^{b}(\Lambda$-mod $) \longrightarrow D^{b}(\Lambda-\bmod ) / \Lambda$-perf $=\Lambda-\underline{\bmod }($ see $[14$, Theorem 2.1]) has dense image. Therefore, by Lemma 4.2, $\operatorname{dim} \Lambda-\underline{\bmod } \leq$ $\operatorname{dim}_{D^{b}(\Lambda-\bmod )} \Lambda$-mod.

We want to use the rest of this section to prove some results on the strength of the inequalities obtained.

4.10 Lemma. Let $\Lambda$ be a non-semisimple finite dimensional algebra. Then $\Lambda$ is representation finite if and only if $\operatorname{dim} \Lambda-\bmod =0$.

Proof. If $\Lambda$ is representation finite then $\Lambda-\bmod \subset\langle M\rangle$ for $M$ an additive generator of $\Lambda$-mod. If $\Lambda$ is not representation finite, then for any $M \in$ $D^{b}(\Lambda$-mod $)$ there is an indecomposable $\Lambda$-module $X$ which is not a direct summand of a shift of $M$. Therefore $X \notin\langle M\rangle$.

4.11 Corollary. Let $\Lambda$ be a non-semisimple finite dimensional algebra with repdim $\Lambda<4$. Then

$$
\operatorname{repdim} \Lambda=\operatorname{dim} \Lambda-\bmod +2 \text {. }
$$

4.12 Remark. There are no examples of algebras known to satisfy

$$
\operatorname{repdim} \Lambda>\operatorname{dim} \Lambda-\bmod +2
$$

4.13 Lemma. Let $\Lambda$ be a finite dimensional algebra. Then

$$
\frac{\operatorname{dim} D^{b}(\Lambda-\bmod )-1}{2} \leq \operatorname{dim} \Lambda-\bmod \leq D^{b}(\Lambda-\bmod ) .
$$


Proof. The second inequality is Lemma 4.2(1). For the first inequality set $n=\operatorname{dim} \Lambda$-mod, and let $M \in D^{b}\left(\Lambda\right.$-mod) such that $\Lambda-\bmod \subset\langle M\rangle_{n+1}$. Assume $X \in C^{b}(\Lambda$-mod). We consider the following short exact sequence of complexes:

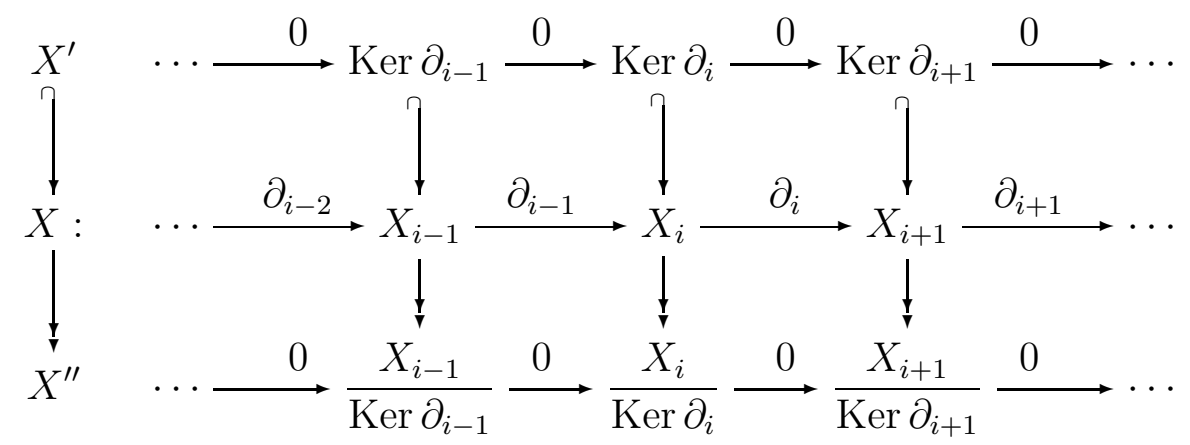

This short exact sequence induces a triangle in the derived category. Clearly $X^{\prime}, X^{\prime \prime} \in\langle\Lambda$-mod $\rangle=\langle M\rangle_{n+1}$. Therefore $X \in\langle M\rangle_{2 n+2}$. Since this works for any $X$, we have $\operatorname{dim} D^{b}(\Lambda$-mod $) \leq 2 n+1$.

4.14 Proposition. Assume $\Lambda$ is a finite dimensional algebra of finite global dimension. If $\operatorname{dim} \Lambda-\bmod \leq 1$ then wresol.dim $\Lambda-\bmod \leq 1$.

Proof. Since gld $\Lambda<\infty$ we may identify $D^{b}(\Lambda$-mod $)=K^{b}(\Lambda$-proj $)$. Let $M \in K^{b}\left(\Lambda\right.$-proj) such that $\Lambda$-mod $\subset\langle M\rangle_{2}$ (note that modules are replaced by their projective resolution when we do the above identification), say $M$ has the form

$$
M=\cdots \stackrel{\partial_{i-1}}{\longrightarrow} M_{i-1} \stackrel{\partial_{i}}{\longrightarrow} M_{i} \stackrel{\partial_{i+1}}{\longrightarrow} M_{i+1} \stackrel{\partial_{i+2}}{\longrightarrow} \cdots .
$$

Set $N=\left(\oplus_{i} M_{i} / \operatorname{Im} \partial_{i}\right) \oplus \Lambda$. We claim that for any $X \in \Lambda$-mod the weak $N$-resolution dimension of $X$ is at most 1 . By assumption, there is a triangle $M^{\prime} \longrightarrow M^{\prime \prime} \longrightarrow X \longrightarrow M^{\prime}[1]$ in $K^{b}(\Lambda$-proj). We may assume (possibly adding complexes of the form $0 \longrightarrow P \stackrel{1}{\longrightarrow} P \longrightarrow 0$ with $P \in$ add $\Lambda$ ) that this triangle comes from a short exact sequence of complexes. We naively cut off everything in degrees $\geq 1$. Then we have the following setup:

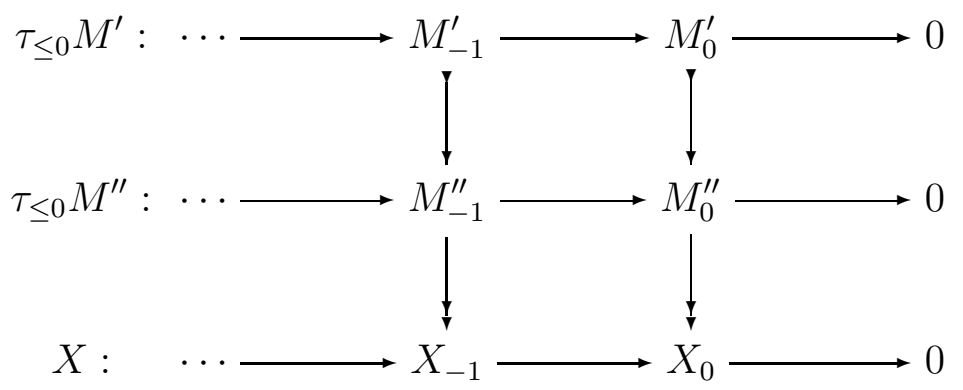


Applying $\operatorname{Hom}_{K^{b}(\Lambda \text {-proj })}(\Lambda,-)$ we obtain an exact sequence of $\Lambda$-modules

$$
M_{0}^{\prime} / \operatorname{Im} \partial_{0}^{\prime} \longrightarrow M_{0}^{\prime \prime} / \operatorname{Im} \partial_{0}^{\prime \prime} \longrightarrow X
$$

since $X$ is exact at position -1 and has homology $X$ at position 0 . This way we obtain a weak $N$-resolution of length 1 for any $X \in \Lambda$-mod. Hence wresol.dim $\Lambda-\bmod \leq 1$.

4.15 Corollary. Assume $\Lambda$ is a finite dimensional algebra of finite global dimension, with wresol.dim $\Lambda-\bmod <3$. Then

$$
\text { wresol.dim } \Lambda-\bmod =\operatorname{dim} \Lambda-\bmod .
$$




\section{A criterion for finding lower bounds for the dimension of module categories}

The aim of this section is to prove a criterion which yields lower bounds for the dimension of a given module category, and hence by 4.6 also for the representation dimension and the dimension of the corresponding algebra. In the first subsection we give and prove a general version of this criterion. In the second subsection we further investigate one special case. This will be the one which is easiest to apply. In Subsection 5.3 we will see that the assumptions of the criterion are preserved under certain coverings. Therefore we immediately get the same lower bounds for the dimension of the module categories of related algebras.

\subsection{The general criterion}

One main idea of the criterion presented here is to compare the (derived categories of) the module category we are interested in to another category we understand better. We will use the category of modules over an integral domain which is finitely generated as $k$-algebra and its derived category as this other category. Here we can apply results from 2.3. We first need to extend these ideas to the derived category.

\section{Vanishing of extensions over $k\left[x_{1}, \ldots x_{d}\right] / I$}

We fix a field $k$ and $R=k\left[x_{1}, \ldots x_{d}\right] / I$ with $I \triangleleft k\left[x_{1}, \ldots, x_{d}\right]$ a prime ideal. We denote by $R$-f.l. the category of $R$-modules of finite length. One main idea here is to look at a family of objects in $D^{b}(\Lambda$-mod) by taking a complex $G$ of $\Lambda \otimes_{k} R$-lattices and looking at the image of the functor

$$
G \otimes_{R}-: D^{b}(R-\text { f.l. }) \longrightarrow D^{b}(\Lambda-\bmod ) \text {. }
$$

The first aim now is to recall some properties of $R$-f.l., which will then be used to study the image of $G \otimes_{R}-$ in $D^{b}(\Lambda$-mod). More precisely, we will prove Proposition 5.1.1, which says that for any $M \in D^{-}(R$-mod) there is an open subset of blocks of $R$-f.l., such that for any block in this open subset the homomorphisms from $M$ to this block annihilate all extensions in the block.

We denote by MaxSpec $R$ the set of maximal ideals of $R$, with Zariski topology. For $\mathfrak{p} \in$ MaxSpec $R$ we denote by $R_{\mathfrak{p}}-$ f.l. the category of modules of finite length over the localization of $R$ at $\mathfrak{p}$. This is the full subcategory 
of $R$-f.l. whose the objects are all iterated extensions of the simple module $R / \mathfrak{p}$. This yields a block decomposition

$$
R \text {-f.l. }=\bigoplus_{\mathfrak{p} \in \operatorname{MaxSpec} R} R_{\mathfrak{p}} \text {-f.l. }
$$

5.1.1 Proposition. Let $M \in D^{-}(R$-mod). There is a non-empty open set $\mathcal{U} \subset \operatorname{MaxSpec} R$ such that for any $\mathfrak{p} \in \mathcal{U}$ and any $X_{1}, X_{2} \in R_{\mathfrak{p}}-\bmod$

$$
\operatorname{Hom}_{D^{-}(R \text {-mod })}\left(M, X_{1}\right) \operatorname{Hom}_{D^{-}(R-\text { mod })}\left(X_{1}, X_{2}[1]\right)=0 .
$$

Proof. Since $D^{-}(R$-mod $)=K^{-}(R$-proj $)$ we may assume that $M$ is a complex of projectives. Then $\operatorname{Hom}_{D^{-}(R \text {-mod })}(M, X)=\operatorname{Hom}_{K^{-}(R \text {-mod })}(M, X)$ for any $X \in D\left(R\right.$-mod). Any morphism from $M$ to $X_{1}$ factors through $\tau_{\geq 0} M$, where $\tau_{\geq 0} M$ is the truncated complex as illustrated in the following diagram.

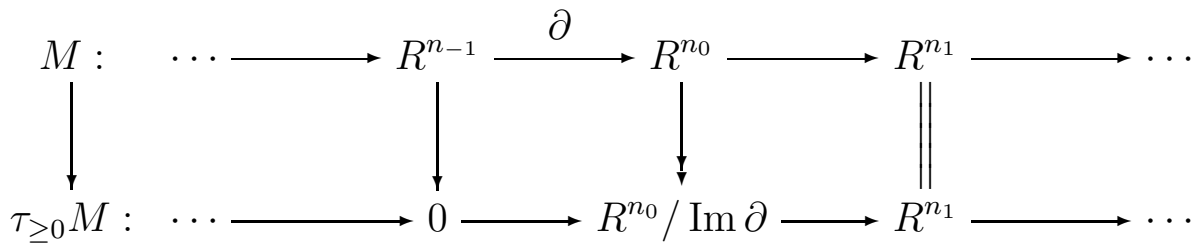

Further, since $X_{1}$ in an $R_{\mathfrak{p}}$-module, any map $\tau_{\geq 0} M \longrightarrow X_{1}$ factors through $R_{\mathfrak{p}} \otimes_{R} \tau_{\geq 0} M$. By Lemma 2.3.1 there is a non-empty open subset $\mathcal{U} \subseteq$ MaxSpec $R$ such that $R_{\mathfrak{p}} \otimes_{R} R^{n_{0}} / \operatorname{Im} \partial$ is projective (as $R_{\mathfrak{p}}$-modules) for any $\mathfrak{p} \in \mathcal{U}$. Then clearly $\operatorname{Hom}_{D^{-}\left(R_{\mathfrak{p}} \text {-mod }\right)}\left(R_{\mathfrak{p}} \otimes_{R} \tau_{\geq 0} M, X_{2}[1]\right)=0$. The claim of the proposition now follows from the fact that the embedding

$$
D^{-}\left(R_{\mathfrak{p}}-\bmod \right) \longrightarrow D^{-}(R-\operatorname{Mod})
$$

is full.

\section{The criterion}

Now we can state and prove our main theorem. We keep $R$ fixed as in the paragraph above, and also fix a finite dimensional $k$-algebra $\Lambda$.

Assume further we are in the following situation:

5.1.2 Setup. We have a functor $\mathrm{F}: D^{b}(R$-f.l. $) \longrightarrow K^{b}(\Lambda$-inj $)$ such that the image of $R$-f.l. [i] is uniformly bounded for any $i$, that is, $\mathrm{F}$ restricts to functors $R$-f.l. $[i] \longrightarrow K^{\left[a_{1}^{i}, a_{2}^{i}\right]}\left(\Lambda\right.$-inj), where $K^{\left[a_{1}^{i}, a_{2}^{i}\right]}$ denotes the subcategory of complexes which vanish outside degrees $a_{1}^{i}, \ldots, a_{2}^{i}$. Assume further that $\mathrm{F}$ admits a left adjoint $\widetilde{\mathrm{F}}: D^{b}(\Lambda$-mod $) \longrightarrow D^{-}(R$-mod $)$. That is, there is a natural isomorphism

$$
\begin{aligned}
& \operatorname{Hom}_{D^{b}(\Lambda \text {-mod })}(M, F X) \cong \operatorname{Hom}_{D^{-}(R \text {-mod })}(\widetilde{\mathrm{F}} M, X) \\
& \forall M \in D^{b}(\Lambda \text {-mod }), X \in D^{b}(R \text {-f.l. }) .
\end{aligned}
$$


5.1.3 Proposition. Let $\mathrm{F}: D^{b}(R$-f.l. $) \longrightarrow K^{b}(\Lambda$-inj $)$ be as described above, $d \in \mathbb{N}$.

(a) Assume

$$
\left\{\mathfrak{p} \in \operatorname{MaxSpec} R \mid \mathrm{F}\left(\operatorname{Hom}_{D^{b}(R \text {-mod })}\left(R_{\mathfrak{p}} \text {-f.l., } R_{\mathfrak{p}} \text {-f.l. }[d]\right)\right) \neq 0\right\}
$$

is dense. Then

$$
\operatorname{dim} D^{b}(\Lambda-\bmod ) \geq d
$$

(b) Assume

$$
\begin{aligned}
& \left\{\mathfrak{p} \in \operatorname{MaxSpec} R \mid \mathrm{F}\left(\operatorname{Hom}_{D^{b}(R-\mathrm{mod})}\left(R_{\mathfrak{p}}-\mathrm{f} .1 ., R_{\mathfrak{p}}-\mathrm{f} . \mathrm{l} .[d]\right)\right)\right. \text { contains } \\
& \text { at least one map of complexes which is not locally null-homotopic }\}
\end{aligned}
$$

is dense. Then

$$
\operatorname{dim} \Lambda-\bmod \geq d
$$

and especially

$$
\operatorname{repdim} \Lambda \geq d+2
$$

Proof. We want to apply Corollary 2.2.10 and Proposition 4.8 for claim (a) and claim (b) respectively. Therefore let $M \in D^{b}(\Lambda$-mod). Assume $M \in$ $D^{\left[b_{1}, b_{2}\right]}\left(\Lambda\right.$-mod). We set $a_{1}=\min \left\{a_{1}^{i} \mid 0 \leq i \leq d-1\right\}, a_{2}=\max \left\{a_{2}^{i} \mid 0 \leq\right.$ $i \leq d-1\}$, and

$$
\widehat{M}=\bigoplus_{i=b_{2}-a_{1}}^{a_{2}-b_{1}} M[i] .
$$

That is we take the direct sum of all shifts of $M$, excluding those which cannot have any morphisms to objects in $\mathrm{F}(R$-f.l.[i]) for any $i \in\{1, \ldots, d-1\}$. We apply Proposition 5.1.1 to

$$
\bigoplus_{i=0}^{d-1} \widetilde{\mathrm{F}}(\widehat{M})[-i]
$$

This yields a non-empty open $\mathcal{U} \subset$ MaxSpec $R$. Choose $\mathfrak{p}$ in the intersection of $\mathcal{U}$ with the subset of MaxSpec $R$ described in the proposition (this is possible by assumption).

Now choose an element $f$ of $\operatorname{Hom}_{D^{b}(R \text {-mod })}\left(R_{\mathfrak{p}}-\right.$ f.l., $R_{\mathfrak{p}}-$ f.l. $\left.[d]\right)$ which is not mapped to 0 by $\mathrm{F}$. For the proof of (b) choose $f$ such that $\mathrm{F} f$ is not locally null-homotopic. Decompose it into a product

$$
f=f_{1} \cdot\left(f_{2}[1]\right) \cdots\left(f_{d}[d-1]\right)
$$


with $f_{i} \in \operatorname{Hom}_{D^{b}(R \text {-mod })}\left(R_{\mathfrak{p}}\right.$-f.l., $R_{\mathfrak{p}}$-f.l.[1]), say $f_{i}: X_{i-1} \longrightarrow X_{i}[1] . \quad$ By assumption on $\mathfrak{p}$ we have

$$
\operatorname{Hom}_{D^{-}(R \text {-mod })}\left(\widetilde{\mathrm{F}}(\widehat{M})[-(i-1)], X_{i-1}\right) \cdot f_{i}=0 .
$$

Therefore also

$$
\operatorname{Hom}_{D^{-}(R \text {-mod })}\left(\widetilde{\mathrm{F}}(\widehat{M}), X_{i-1}[i-1]\right) \cdot f_{i}[i-1]=0 .
$$

Now we apply $\mathrm{F}$ to the shift of $f_{i}$ and the adjunction isomorphism to the Hom-set. That yields

$$
\operatorname{Hom}_{D^{b}(\Lambda \text {-mod })}\left(\widehat{M}, \mathrm{~F}\left(X_{i-1}[i-1]\right)\right) \cdot \mathrm{F}\left(f_{i}[i-1]\right)=0 .
$$

By construction of $\widehat{M}$ this means

$$
\operatorname{Hom}_{D^{b}(\Lambda \text {-mod })}\left(M[j], \mathrm{F}\left(X_{i-1}[i-1]\right)\right) \cdot \mathrm{F}\left(f_{i}[i-1]\right)=0 \quad \forall j .
$$

Now apply Corollary 2.2.10 for the proof of (a) and Proposition 4.8 for the proof of (b).

5.1.4 Definition. We define $\Lambda \otimes_{k} R$-lat to be the full subcategory of $\Lambda \otimes_{k}$ $R$-mod in which the objects are projective as $R$-modules. We denote by $\operatorname{Inj}\left(\Lambda \otimes_{k} R\right.$-lat $)$ the full subcategory of objects, which are injective with respect to short exact sequences (that is, any short exact sequence which begins in such an object splits).

Note that $\operatorname{Inj}\left(\Lambda \otimes_{k} R\right.$-lat $)$ contains all modules of the form $I \otimes_{k} R$, with $I \in \Lambda$-inj.

An object $G \in C^{b}\left(\operatorname{Inj}\left(\Lambda \otimes_{k} R\right.\right.$-lat $\left.)\right)$ gives rise to a functor

$$
G \otimes_{R}^{\mathrm{L}}-: D^{b}(R \text {-f.l. }) \longrightarrow D^{b}(\Lambda-\bmod ) .
$$

Since $G$ consists of projective $R$-modules this is just the total tensor product $G \otimes_{R}^{\mathrm{L}}-=G \otimes_{R}^{\mathrm{Tot}}-$.

Theorem 2. Let $G \in C^{b}\left(\operatorname{Inj}\left(\Lambda \otimes_{k} R\right.\right.$-lat $\left.)\right)$ and $d \in \mathbb{N}$.

(a) Assume

$$
\left\{\mathfrak{p} \in \operatorname{MaxSpec} R \mid\left(G \otimes_{R}^{\text {Tot }}-\right)\left(\operatorname{Hom}_{D^{b}(R \text {-mod })}\left(R_{\mathfrak{p}} \text {-f.l., } R_{\mathfrak{p}} \text {-f.l. }[d]\right)\right) \neq 0\right\}
$$

is dense. Then

$$
\operatorname{dim} D^{b}(\Lambda-\bmod ) \geq d
$$


(b) Assume

$\left\{\mathfrak{p} \in \operatorname{MaxSpec} R \mid\left(G \otimes_{R}^{\text {Tot }}-\right)\left(\operatorname{Hom}_{D^{b}(R \text {-mod })}\left(R_{\mathfrak{p}}-\right.\right.\right.$ f.l., $R_{\mathfrak{p}}-\mathrm{f}$.l. $\left.[d]\right)$ contains at least one map of complexes which is not locally null-homotopic\}

is dense. Then

$$
\operatorname{dim} \Lambda-\bmod \geq d
$$

and especially

$$
\operatorname{repdim} \Lambda \geq d+2
$$

Proof. Clearly we want to apply Proposition 5.1.3 with $\mathrm{F}=G \otimes_{R}^{\text {Tot }}-$. It only remains to show that $\mathrm{F}$ has a left adjoint.

Since $G$ is finitely generated and projective over $R$ it is isomorphic to $\operatorname{Hom}_{R}^{\text {Tot }}\left(\operatorname{Hom}_{R}^{\text {Tot }}(G, R), R\right)$ (note that applying $\operatorname{Hom}_{R}^{\text {Tot }}(-, R)$ just means applying $\operatorname{Hom}_{R}(-, R)$ to every degree). Therefore we have

$$
\begin{aligned}
\operatorname{Hom}_{D^{b}(\Lambda \text {-mod })} & \left(M, G \otimes_{R}^{\text {Tot }} X\right) \\
& \cong \operatorname{Hom}_{D^{b}(\Lambda \text {-mod })}\left(M, \operatorname{Hom}_{R}^{\text {Tot }}\left(\operatorname{Hom}_{R}^{\text {Tot }}(G, R), R\right) \otimes_{R}^{\text {Tot }} X\right) \\
& \cong \operatorname{Hom}_{D^{b}(\Lambda \text {-mod })}\left(M, \operatorname{Hom}_{R}^{\text {Tot }}\left(\operatorname{Hom}_{R}^{\text {Tot }}(G, R), X\right)\right) \\
& \cong \operatorname{Hom}_{D^{-}(R \text {-mod })}\left(\operatorname{Hom}_{R}^{\text {Tot }}(G, R) \otimes_{\Lambda}^{L} M, X\right)
\end{aligned}
$$

So $\operatorname{Hom}_{R}^{\text {Tot }}(G, R) \otimes_{\Lambda}^{L}-$ is the desired adjoint.

5.1.5 Remark. Since $\Lambda$-inj $\approx \Lambda$-proj we may in Theorem 2 alternatively assume $G \in \Lambda \otimes_{k} R$-proj.

Let us now assume that $L \in \Lambda \otimes_{k} R$-lat. Then $\left(L \otimes_{R}-\right)$ is an exact functor $R$-f.l. $\longrightarrow \Lambda$-mod. Therefore it also induces maps between corresponding Ext-groups.

5.1.6 Corollary. Let $L$ be a $\Lambda \otimes R$-lattice, and let $d \in \mathbb{N}$. Assume the set

$$
\left\{\mathfrak{p} \in \operatorname{MaxSpec} R \mid\left(L \otimes_{R}-\right)\left(\operatorname{Ext}_{R}^{d}\left(R_{\mathfrak{p}}-\text { f.l., } R_{\mathfrak{p}}-\text { f.l. }\right)\right) \neq 0\right\}
$$

is dense. Then

$$
\operatorname{dim} \Lambda-\bmod \geq d
$$

and in particular

$$
\operatorname{repdim} \Lambda \geq d+2 .
$$


Proof. Let $L$ satisfy the assumption above, and let $\mathfrak{p} \in \operatorname{MaxSpec} R$ such that $\left(L \otimes_{R}-\right)\left(\operatorname{Ext}_{R}^{d}\left(R_{\mathfrak{p}}\right.\right.$-f.l., $R_{\mathfrak{p}}$-f.l. $\left.)\right) \neq 0$. Then there are $A, B \in R_{\mathfrak{p}}$-f.l. and $\mathbb{E} \in \operatorname{Ext}_{R}^{d}(A, B)$ such that $L \otimes_{R} \mathbb{E} \neq 0$ as element of $\operatorname{Ext}_{\Lambda}^{d}\left(L \otimes_{R} A, L \otimes_{R} B\right)$. In the derived category $L$ is isomorphic to a complex of injective lattices of the form $I \otimes_{k} R$, say

$$
L=\cdots \longrightarrow 0 \longrightarrow I^{0} \otimes_{k} R \longrightarrow I^{1} \otimes_{k} R \longrightarrow \cdots
$$

Then

$$
\begin{aligned}
& L \otimes_{R} A=\cdots \longrightarrow 0 \longrightarrow I^{0} \otimes_{k} A \longrightarrow I^{1} \otimes_{k} A \longrightarrow \cdots \\
& L \otimes_{R} B=\cdots \longrightarrow I^{0} \otimes_{k} B \longrightarrow I^{1} \otimes_{k} B \longrightarrow \cdots
\end{aligned}
$$

and the extension is induced by a map of complexes

$$
L \otimes_{R} A \longrightarrow L \otimes_{R} B[d]
$$

Assume it is locally null-homotopic in position 0. Then, since we have complexes of injectives which are exact in all other positions, this local nullhomotopy can be lifted to a null-homotopy. Therefore the extension splits, contradicting our assumption. Therefore there is also no such local nullhomotopy in position 0 for $G \otimes_{R} \mathbb{E}$, with

$$
G=\cdots \longrightarrow 0 \longrightarrow I^{0} \otimes_{k} R \longrightarrow \cdots \longrightarrow I^{d} \otimes_{k} R \longrightarrow 0 \longrightarrow \cdots
$$

Now we apply Theorem 2 with this $G$.

\subsection{A practical version of the criterion}

In this subsection we will treat the following special case: We assume $R=$ $k\left[x_{1}, \ldots, x_{d}\right]$ and $G$ is a complex of injectives such that the differential is a polynomial of degree one. This setup will be used in the examples.

We denote by $\bar{k}$ the algebraic closure of $k$. The inclusion

$$
k\left[x_{1}, \ldots, x_{d}\right] \subset \bar{k}\left[x_{1}, \ldots, x_{d}\right]
$$

induces a surjection

$$
\zeta: \bar{k}^{d}=\operatorname{MaxSpec} \bar{k}\left[x_{1}, \ldots, x_{d}\right] \longrightarrow \operatorname{MaxSpec} k\left[x_{1}, \ldots, x_{d}\right]
$$

In particular the $\zeta$-image of dense subsets is dense.

For $\left(\alpha_{1}, \ldots, \alpha_{d}\right) \in \operatorname{MaxSpec} k\left[x_{1}, \ldots x_{d}\right]$, we denote by $\widehat{k}=k\left[\alpha_{1}, \ldots, \alpha_{d}\right]$ the corresponding finite extension of $k$. 
5.2.1 Corollary. Let $R=k\left[x_{1}, \ldots, x_{d}\right]$. Assume $G \in C^{b}\left(\operatorname{Inj}\left(\Lambda \otimes_{k} R\right.\right.$-lat $\left.)\right)$ is of the form

$$
I^{0} \otimes_{k} R \stackrel{\partial_{0}^{1}+\sum_{i=1}^{d} \partial_{i}^{1} x_{i}}{\longrightarrow} I^{1} \otimes_{k} R \stackrel{\partial_{0}^{2}+\sum_{i=1}^{d} \partial_{i}^{2} x_{i}}{\longrightarrow} \cdots \stackrel{\partial_{0}^{d}+\sum_{i=1}^{d} \partial_{i}^{d} x_{i}}{\longrightarrow} I^{d} \otimes_{k} R,
$$

with $I^{i} \in \Lambda$-inj and $\partial_{i}^{j} \in \operatorname{Hom}_{\Lambda}\left(I^{j-1}, I^{j}\right)$. Assume the set

$$
\begin{aligned}
& \left\{\left(\alpha_{1}, \ldots \alpha_{d}\right) \in \bar{k}^{d} \mid\right. \text { the map } \\
& \begin{array}{l}
I^{0} \otimes_{k} \widehat{k} \stackrel{\partial_{0}^{1}+\sum_{i=1}^{d} \partial_{i}^{1} \alpha_{i}}{\longrightarrow} I^{1} \otimes_{k} \widehat{k} \\
\downarrow_{\partial_{1}^{1} \cdots \partial_{d}^{d}} \\
I^{d-1} \otimes_{k} \widehat{k} \stackrel{\partial_{0}^{d}+\sum_{i=1}^{d} \partial_{i}^{d} \alpha_{i}}{\longrightarrow} I^{d} \otimes_{k} \widehat{k} \\
\text { is not null-homotopic }\}
\end{array}
\end{aligned}
$$

is Zariski-dense in $\bar{k}^{d}$. Then

$$
\operatorname{dim} \Lambda-\bmod \geq d
$$

Proof. We only need to show that we are in the situation of Theorem 2(b). Assume $\left(\alpha_{1}, \ldots, \alpha_{d}\right)$ is in the set above. We consider the exact sequences

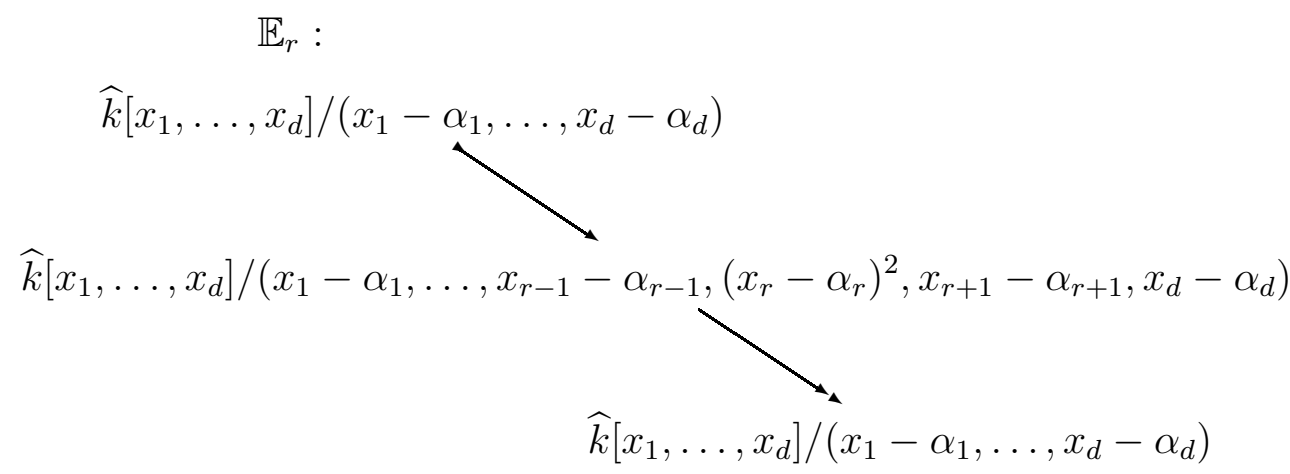

of $R$-modules, where the first map is the $\widehat{k}\left[x_{1}, \ldots, x_{n}\right]$-linear map sending 1 to $x_{r}-\alpha_{r}$, and the second map is projection. Tensoring $\mathbb{E}_{r}$ with $G$ we find 
the following short exact sequence of complexes of $\Lambda$-modules.

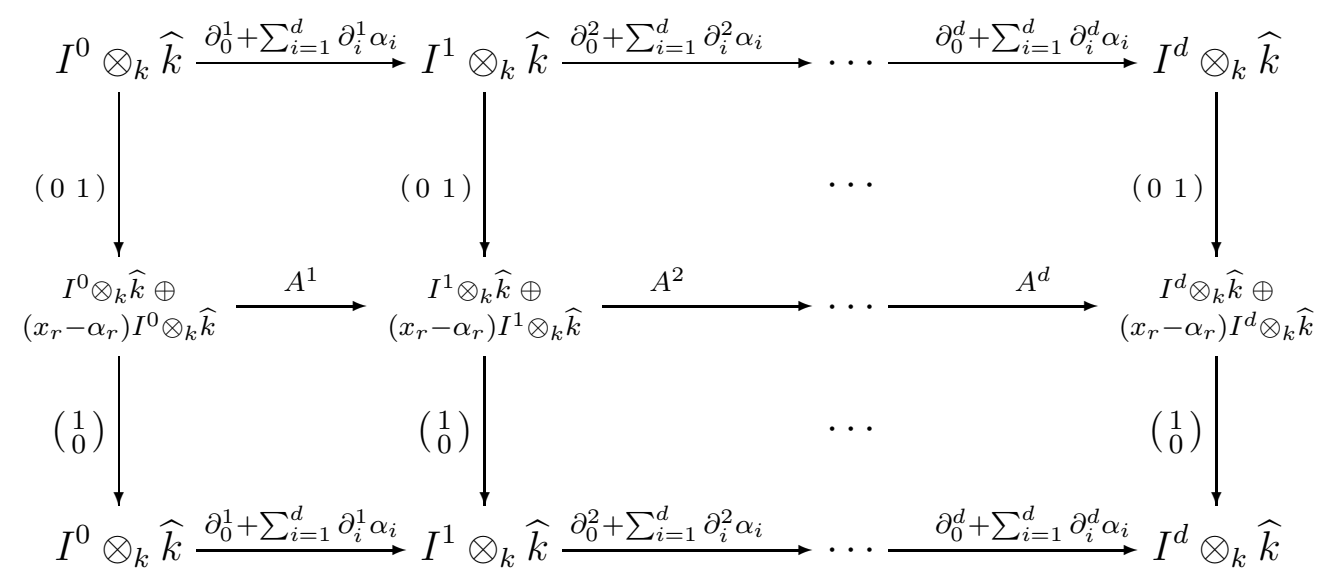

with $A^{j}=\left(\begin{array}{cc}\partial_{0}^{j}+\sum_{i=1}^{d} \partial_{i}^{j} \alpha_{i} & \partial_{r}^{j} \\ 0 & \partial_{0}^{j}+\sum_{i=1}^{d} \partial_{i}^{j} \alpha_{i}\end{array}\right)$. The map in the homotopy category corresponding to this extension is

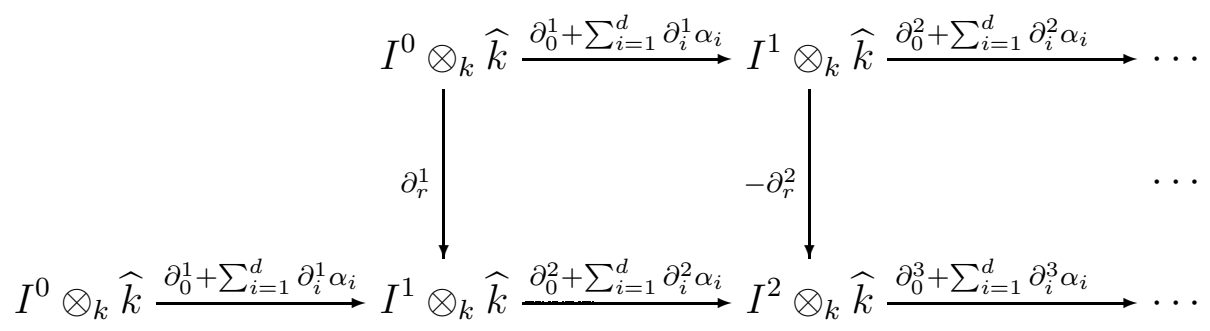

Now we look at the composition $\mathbb{E}_{1} \cdots \mathbb{E}_{d} \in \operatorname{Ext}_{R}^{d}$. By assumption it is not locally null-homotopic. Therefore $\zeta\left(\alpha_{1}, \ldots, \alpha_{d}\right)$ is in the set

$\left\{\mathfrak{p} \in \operatorname{MaxSpec} R \mid\left(G \otimes_{R}^{\text {Tot }}-\right)\left(\operatorname{Hom}_{D^{b}(R \text {-mod })}\left(R_{\mathfrak{p}}\right.\right.\right.$-f.l., $R_{\mathfrak{p}}$-f.l. $\left.[d]\right)$ contains at least one map of complexes which is not locally null-homotopic\}.

Therefore this set is dense, so the assumption of Theorem 2(b) is satisfied.

Now we can reformulate Corollary 5.2.1 in a way which does not contain the $R$-lattice structure explicitly any more, but only requires us to find a finite set of morphisms between injective $\Lambda$-modules having certain properties.

5.2.2 Proposition. For $0 \leq j \leq d$ let $I^{j} \in \Lambda$-inj and for $0 \leq i \leq d$ and $0<j \leq d$ let $\partial_{i}^{j} \in \operatorname{Hom}_{\Lambda}\left(I^{j-1}, I^{j}\right)$, such that

(1) $\forall i, j: \partial_{i}^{j} \partial_{i}^{j+1}=0$ and

(2) $\forall i_{1}, i_{2}, j: \partial_{i_{1}}^{j} \partial_{i_{2}}^{j+1}=-\partial_{i_{2}}^{j} \partial_{i_{1}}^{j+1}$. 
Assume the set

$$
\begin{gathered}
\left\{\left(\alpha_{1}, \ldots \alpha_{d}\right) \in \bar{k}^{d} \mid \text { for } \widehat{k}=k\left[\alpha_{1}, \ldots \alpha_{d}\right]\right. \text { the map } \\
\qquad I^{0} \otimes_{k} \widehat{k} \stackrel{\partial_{0}^{1}+\sum_{i=1}^{d} \partial_{i}^{1} \alpha_{i}}{\longrightarrow} I^{1} \otimes_{k} \widehat{k} \\
\\
I^{d-1} \otimes_{k} \widehat{k} \stackrel{\partial_{0}^{d}+\sum_{i=1}^{d} \partial_{i}^{d} \alpha_{i}}{\longrightarrow} I^{d} \otimes_{k} \widehat{k} \\
\text { is not null-homotopic }\}
\end{gathered}
$$

is Zariski-dense in $\bar{k}^{d}$. Then

$$
\operatorname{dim} \Lambda-\bmod \geq d
$$

Proof. We apply Corollary 5.2.1 to the complex

$$
I^{0} \otimes_{k} R \stackrel{\partial_{0}^{1}+\sum_{i=1}^{d} \partial_{i}^{1} x_{i}}{\longrightarrow} I^{1} \otimes_{k} R \stackrel{\partial_{0}^{2}+\sum_{i=1}^{d} \partial_{i}^{2} x_{i}}{\longrightarrow} \cdots \stackrel{\partial_{0}^{d}+\sum_{i=1}^{d} \partial_{i}^{d} x_{i}}{\longrightarrow} I^{d} \otimes_{k} R .
$$

Assumptions (1) and (2) of the proposition ensure that this is indeed a complex, that is that the composition of two consecutive morphisms vanishes.

5.2.3 Remark. Note that, in Proposition 5.2.2 above, we have to find out if a morphism of complexes of $\Lambda \otimes_{k} \widehat{k}$-modules is null-homotopic as a map of complexes of $\Lambda$-modules. This seems to be a quite unnatural question. Next we will see that for $\widehat{k}$ separable over $k$ this simplifies to the question whether the map is null-homotopic as a map of complexes of $\Lambda \otimes_{k} \widehat{k}$-modules.

5.2.4 Lemma. Let $\widehat{k}$ be a separable extension of $k$. A map of complexes of $\Lambda \otimes_{k} \widehat{k}$-modules is (locally) null-homotopic as map of complexes of $\Lambda$-modules if and only if it is (locally) null-homotopic as a map of complexes of $\Lambda \otimes_{k} \widehat{k}$ modules.

Proof. The "if"-part is clear.

For the converse let the complexes be $\left(A_{i}\right)$ and $\left(B_{i}\right)$. Assume that there is a $\Lambda$-null-homotopy by maps $h_{i}: A_{i} \longrightarrow B_{i-1}$.

Since $\widehat{k}$ is separable over $k$ the epimorphism $\widehat{k} \otimes_{k} \widehat{k} \stackrel{\pi}{\longrightarrow} \widehat{k}$ of $\widehat{k}-\widehat{k}$-bimodules splits $\left(\left[7\right.\right.$, Corollary 69.8]). Let $\iota: \widehat{k} \longrightarrow \widehat{k} \otimes_{k} \widehat{k}$ be a morphism of $\widehat{k}-\widehat{k}$ bimodules such that $\iota \pi=1$. This induces maps of $\Lambda \otimes_{k} \widehat{k}$ modules

$$
A_{i} \otimes_{k} \widehat{k} \underset{1_{A_{i}} \otimes_{\widehat{k}} \pi}{1_{A_{i}} \otimes_{\widehat{k}} \iota} A_{i}
$$


and similar for $B_{i}$.

Now we replace $h_{i}$ by

$$
\tilde{h}_{i}: A_{i} \stackrel{1_{A_{i}} \otimes_{\widehat{k}} \iota}{\longrightarrow} A_{i} \otimes_{k} \widehat{k} \stackrel{h \otimes_{k} 1_{\widehat{k}}}{\longrightarrow} B_{i-1} \otimes_{k} \widehat{k} \stackrel{1_{B_{i-1}} \otimes_{\widehat{k}} \pi}{\longrightarrow} B_{i-1} .
$$

Note that if $f: X \longrightarrow Y$ is a $\Lambda \otimes_{k} \widehat{k}$-linear map, then $f\left(1_{Y} \otimes_{\widehat{k}} \iota\right)=$ $\left(1_{X} \otimes_{\widehat{k}} \iota\right)\left(f \otimes_{k} 1_{\widehat{k}}\right)$ and $\left(1_{X} \otimes_{\widehat{k}} \pi\right) f=\left(f \otimes_{k} 1_{\widehat{k}}\right)\left(1_{Y} \otimes_{\widehat{k}} \pi\right)$. Using this, it is a straightforward calculation to see that the $\widetilde{h}_{i}$ also induce a null-homotopy.

The proof for locally null-homotopic is similar.

We denote by $k^{\text {sep }}$ the separable closure of $k$. Note that $\left(k^{\text {sep }}\right)^{d}$ is always dense in $\bar{k}^{d}$. Then we obtain the following theorem directly from Proposition 5.2.2 and Lemma 5.2.4.

Theorem 3. For $0 \leq j \leq d$ let $I^{j} \in \Lambda$-inj and for $0 \leq i \leq d$ and $0<j \leq d$ let $\partial_{i}^{j} \in \operatorname{Hom}_{\Lambda}\left(I^{j-1}, I^{j}\right)$, such that

(1) $\forall i, j: \partial_{i}^{j} \partial_{i}^{j+1}=0$ and

(2) $\forall i_{1}, i_{2}, j: \partial_{i_{1}}^{j} \partial_{i_{2}}^{j+1}=-\partial_{i_{2}}^{j} \partial_{i_{1}}^{j+1}$.

Assume the set

$$
\begin{aligned}
& \left\{\left(\alpha_{1}, \ldots \alpha_{d}\right) \in\left(k^{\mathrm{sep}}\right)^{d} \mid \text { for } \widehat{k}=k\left[\alpha_{1}, \ldots \alpha_{d}\right]\right. \text { the map } \\
& \qquad I^{0} \otimes_{k} \widehat{k} \stackrel{\partial_{0}^{1}+\sum_{i=1}^{d} \partial_{i}^{1} \alpha_{i}}{\longrightarrow} I^{1} \otimes_{k} \widehat{k} \\
& \\
& \quad I^{d-1} \otimes_{k} \widehat{k} \stackrel{\partial_{0}^{d}+\sum_{i=1}^{d} \partial_{i}^{d} \alpha_{i}}{\longrightarrow} I^{d} \otimes_{k} \widehat{k} \\
& \\
& \text { is not null-homotopic as map of complexes over } \left.\Lambda \otimes_{k} \widehat{k}\right\}
\end{aligned}
$$

is Zariski-dense in $\left(k^{\mathrm{sep}}\right)^{d}$. Then

$$
\operatorname{dim} \Lambda-\bmod \geq d
$$

\subsection{Coverings}

The aim of this subsection is to show that, under certain assumptions, the preconditions of Theorem 2 are invariant under coverings. This result will allow us to transfer results on local algebras to classes of algebras of finite global dimension in the next section. There are many algebras which admit a covering by the same algebra of finite global dimension. This will yield 
larger families (depending on parameters in $k$ rather than just the discrete parameters $L, N$ ) of algebras for which we can find a lower bound for the representation dimension.

We assume $\Lambda$ to be a graded algebra. That means there is an abelian group $A$, such that $\Lambda=\oplus_{a \in A} \Lambda_{a}$ as $k$-vector space and $\Lambda_{a_{1}} \cdot \Lambda_{a_{2}} \subseteq \Lambda_{a_{1}+a_{2}}$.

A graded $\Lambda$-module is a $\Lambda$-module $M$ with a $k$-vector space decomposition $M=\oplus_{a \in A} M_{a}$ such that $\Lambda_{a_{1}} \cdot M_{a_{2}} \subseteq M_{a_{1}+a_{2}}$. Clearly $\Lambda$ itself is a graded $\Lambda$-module. If $M$ is a graded $\Lambda$-modules and $a \in A$, then we denote my $M\langle a\rangle$ the graded $\Lambda$-module with $M\langle a\rangle=M$ as $\Lambda$-modules, but $M\langle a\rangle_{b}=M_{b-a}$. For two graded $\Lambda$-modules $M$ and $N$ we denote by $\operatorname{Hom}_{\mathrm{gr}}^{g}(M, N)$ the set of graded homomorphisms of degree $g$, that is the homomorphisms which map $M_{a}$ to $N_{a+g}$ for all $a \in A$.

Now let $V \subseteq A$ be a finite subset. We can define a finite dimensional algebra $\Lambda_{V}$ by

$$
\Lambda_{V}=\left(\operatorname{End}_{\mathrm{gr}}^{0}\left(\oplus_{v \in V} \Lambda\langle v\rangle\right)\right)^{\mathrm{op}},
$$

Note that $\operatorname{Hom}_{\mathrm{gr}}^{0}(\Lambda\langle v\rangle, \Lambda\langle w\rangle)=\operatorname{Hom}_{\mathrm{gr}}^{w-v}(\Lambda, \Lambda)=\Lambda_{w-v}$. Therefore the algebra $\Lambda_{V}$ is the matrix algebra

$$
\left(\Lambda_{w-v}\right)_{\substack{v \in V \\ w \in V}}
$$

The indecomposable projective $\Lambda_{V}$-modules are in bijection to the pairs $(Q, v)$ with $Q$ an indecomposable projective $\Lambda$-module and $v \in V$, and

$$
\operatorname{Hom}_{\Lambda_{v}}\left(P_{\left(Q_{1}, v_{1}\right)}, P_{\left(Q_{2}, v_{2}\right)}\right)=\operatorname{Hom}_{\mathrm{gr}}^{v_{2}-v_{1}}\left(Q_{1}, Q_{2}\right) .
$$

This gives rise to a faithful functor

$$
\Lambda_{V} \text {-proj } \longrightarrow \Lambda \text {-proj }
$$

and therefore also to faithful functors

$$
\begin{aligned}
C^{b}\left(\Lambda_{V} \text {-proj }\right) & \longrightarrow C^{b}(\Lambda \text {-proj }), \quad \text { and } \\
C^{b}\left(\Lambda_{V} \otimes_{k} R \text {-proj }\right) & \longrightarrow C^{b}\left(\Lambda \otimes_{k} R \text {-proj }\right),
\end{aligned}
$$

which will all be denoted by C.

For $G \in C^{b}\left(\Lambda \otimes_{k} R\right.$-proj) we set

$$
\begin{aligned}
& d(G)=\min \{d \mid \text { the set } \\
& \left\{\mathfrak{p} \in \operatorname{MaxSpec} R \mid\left(G \otimes_{R}-\right)\left(\operatorname{Hom}_{D^{b}(R \text {-mod })}\left(R_{\mathfrak{p}} \text {-f.l., } R_{\mathfrak{p}} \text {-f.l. }[d]\right)\right.\right. \\
& \quad \text { contains at least one map of complexes which is not locally null- } \\
& \text { homotopic }\} \\
& \text { is dense }\}
\end{aligned}
$$

Then Theorem 2(b) can be restated as follows: 
Theorem 1 (b). Assume $G \in \Lambda \otimes_{k} R$-proj. Then $\operatorname{dim} \Lambda$-mod $\geq d(G)$.

Our aim is to show that $d(G)$ does not change under certain coverings. Together with the formulation of Theorem 2(b) above this means that we can often establish the same lower bounds for the dimension of the module category of $\Lambda_{V}$ that we can show for the dimension of $\Lambda$-mod.

5.3.1 Proposition. Assume $G \in C^{b}\left(\Lambda_{V} \otimes_{k} R\right.$-proj). Then $d(\mathrm{C} G)=d(G)$.

Proof. Tensoring with $X \in R$-f.l. commutes with C. Let $X_{1}, X_{2} \in R$-f.l. and $\varphi: X_{1} \longrightarrow X_{2}[d]$. Clearly if the map $G \otimes_{R} X_{1} \longrightarrow G \otimes_{R} X_{2}[d]$ induced by $\varphi$ is locally null-homotopic, then so is its image under $\mathrm{C}$.

The idea for the converse is, that all parts of a local null-homotopy which do not respect the grading can be omitted.

More precisely, assume the map

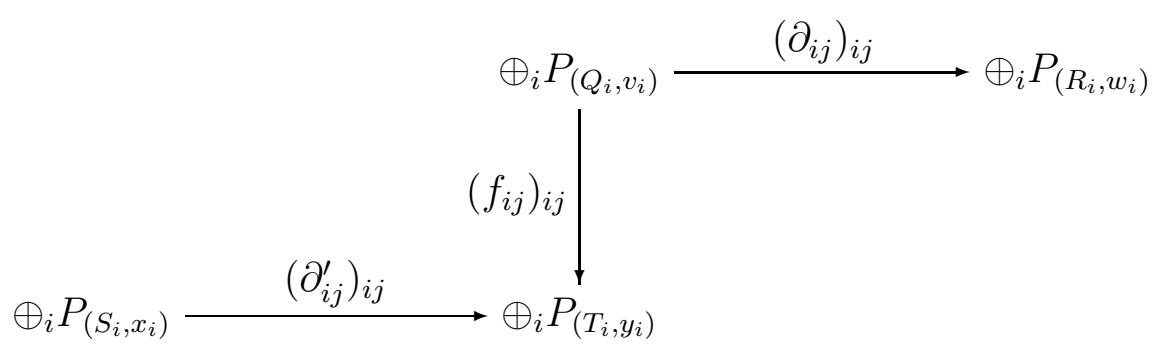

gets null-homotopic by applying C (here $Q_{i}, R_{i}, S_{i}$ and $T_{i}$ are indecomposable projective $\Lambda$-modules and $\left.v_{i}, w_{i}, x_{i}, y_{i} \in V\right)$. We want to show that the map then is null-homotopic itself.

By assumption, there are maps $r_{i j}: Q_{i} \longrightarrow S_{j}$ and $s_{i j}: R_{i} \longrightarrow T_{j}$ as indicated in the following diagram

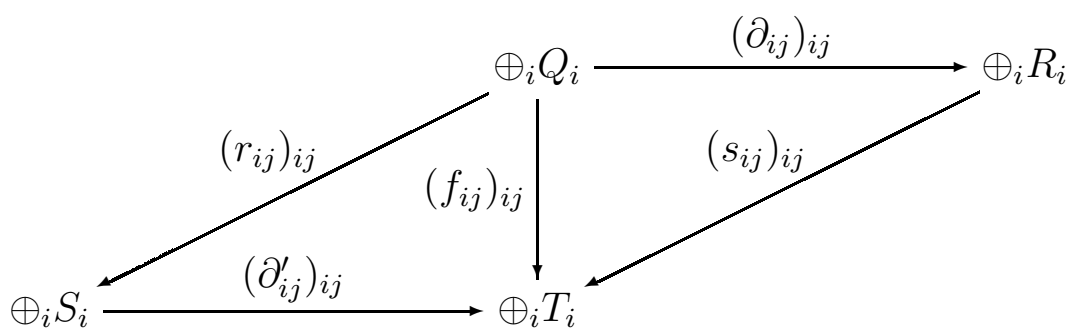

making $f$ null-homotopic.

We can decompose the $r_{i j}$ into $r_{i j}=\sum r_{i j}^{g}$ with $r_{i j}^{g} \in \operatorname{Hom}_{\mathrm{gr}}^{g}\left(Q_{i}, S_{j}\right)$ and the $s_{i j}$ into $s_{i j}=\sum s_{i j}^{g}$ with $s_{i j}^{g} \in \operatorname{Hom}_{\mathrm{gr}}^{g}\left(R_{i}, T_{j}\right)$. New recall that the $f_{i j}, \partial_{i j}$ and $\partial_{i j}^{\prime}$ are graded homomorphisms. Using this fact, it is a straightforward calculation to see that $\left(r_{i j}^{x_{j}-v_{i}}\right)_{i j}$ and $\left(s_{i j}^{y_{j}-w_{i}}\right)_{i j}$ also make $f$ null-homotopic.

The claim now follows from the fact that the $r_{i j}^{x_{j}-v_{i}}$ and $s_{i j}^{y_{j}-w_{i}}$ are in the image of $\mathrm{C}$. 
There can be many graded algebras which have the same covering. We also get the following connection between them:

Assume $\alpha: A \longrightarrow$ Aut $_{\mathrm{gr}} \Lambda$ is a homomorphism of groups. Then we can define a finite dimensional algebra $\Lambda^{\alpha}$ by

$$
\begin{aligned}
& \Lambda^{\alpha} \cong \Lambda \text { as } k \text {-vector spaces, and } \\
& \lambda_{1} \cdot{ }_{\alpha} \lambda_{2}=\lambda_{1}^{\alpha\left(\operatorname{deg} \lambda_{2}\right)} \cdot \lambda_{2} .
\end{aligned}
$$

It is straightforward to verify that $\Lambda^{\alpha}$ is an algebra and that $\Lambda_{V}^{\alpha} \cong \Lambda_{V}$ for any $V \subseteq A$.

By applying Proposition 5.3.1 to both $\Lambda$ and $\Lambda^{\alpha}$ we obtain the following corollary.

5.3.2 Corollary. Let $G \in C^{b}\left(\Lambda \otimes_{k} R\right.$-proj) such that there is some $V \subset A$ and $G^{\prime} \in C^{b}\left(\Lambda_{V} \otimes_{k} R\right.$-proj) with $G=\mathrm{C}_{\Lambda} G^{\prime}$. Then $d\left(\mathrm{C}_{\Lambda^{\alpha}} G^{\prime}\right)=d(G)$. 


\section{Iyama's upper bound for the representa- tion dimension}

The results presented in this section are based on the following theorem of Iyama. The application of his result to the examples was suggested by Iyama, who worked out in detail the upper bound for the representation dimension of the algebra considered by Krause and Kussin presented in the next section as Lemma 7.2.8 (private communication). We recall the proof of Iyama's theorem. Then we give two corollaries, which are adapted to the examples we want to study in the next section.

6.1 Theorem (Iyama [10, Theorem 2.2.2 and Theorem 2.5.1]). Let $\Lambda$ be a finite dimensional algebra. Let $M=M_{0} \in \Lambda-\bmod$ and $M_{i+1}=$ $M_{i} \cdot \operatorname{Rad}\left(\operatorname{End}\left(M_{i}\right)\right)$. Assume $M_{m}=0$. Then

$$
\operatorname{gld} \operatorname{End}\left(\bigoplus_{i} M_{i}\right) \leq m
$$

Especially, for $M=\Lambda \oplus \Lambda^{*}$,

$$
\text { repdim } \Lambda \leq m \text {. }
$$

Proof. We set $\widehat{M}=\oplus_{i} M_{i}$. Note that $M^{\prime} \longmapsto \operatorname{Hom}_{\Lambda}\left(M^{\prime}, \widehat{M}\right)^{*}$ induces an equivalence add $\widehat{M} \longrightarrow \operatorname{End}(\widehat{M})^{\mathrm{op}}$-inj. Let $X \in \operatorname{End}(\widehat{M})^{\mathrm{op}}$-mod and let

$$
X \longrightarrow \operatorname{Hom}_{\Lambda}\left(N^{0}, \widehat{M}\right)^{*} \stackrel{\operatorname{Hom}_{\Lambda}\left(f^{0}, \widehat{M}\right)^{*}}{\longrightarrow} \operatorname{Hom}_{\Lambda}\left(N^{1}, \widehat{M}\right)^{*} \stackrel{\operatorname{Hom}_{\Lambda}\left(f^{1}, \widehat{M}\right)^{*}}{\longrightarrow} \cdots
$$

be a minimal injective coresolution. We will show $N^{i} \in \operatorname{add} \oplus_{j \geq \max \{i-1,0\}} M_{j}$ by induction on $i$. For $i \in\{0,1\}$ there is nothing to show. Assume $i \geq 1$, $N^{i} \in$ add $\oplus_{j \geq i-1} M_{j}$ and $N^{i+1}$ has an indecomposable direct summand $N^{\prime}$ with $N^{\prime} \in$ add $M_{k} \backslash$ add $M_{k+1}$ for some $k<i$. We denote by $\pi: N^{i+1} \longrightarrow N^{\prime}$ the projection to this summand. By assumption there is an epimorphism $\left(M_{k}\right)^{n} \longrightarrow N^{i}$ for some $n \in \mathbb{N}$. The composition of this epimorphism with the component $N^{i} \stackrel{f^{i} \pi}{\longrightarrow} N^{\prime}$ is a radical morphism (since $f^{i}$ is a radical morphism), and therefore factors through $M_{k} \cdot \operatorname{Rad}_{\Lambda \text {-mod }}\left(M_{k}, N^{\prime}\right) \stackrel{\iota}{\longrightarrow} N^{\prime}$. Therefore so does the map $f^{i} \pi$, that is, there is a map $\varphi$ making the following diagram commutative.

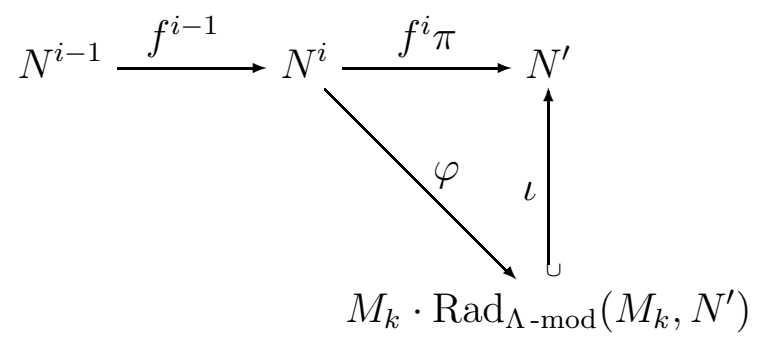


Since $M_{k} \cdot \operatorname{Rad}_{\Lambda-\bmod }\left(M_{k}, N^{\prime}\right) \in$ add $M_{k+1}$ and $N^{\prime} \notin$ add $M_{k+1}$ the embedding $\iota$ is not an isomorphism. Since $\iota$ is an inclusion $f^{i-1} \varphi=0$. This contradicts the assumption that $\operatorname{Hom}\left(f^{i} \pi, \widehat{M}\right)^{*}$ is a component of the minimal injective resolution. Therefore $N^{i+1} \in$ add $\oplus_{j \geq i} M_{j}$, completing our induction.

Since $N^{i} \in$ add $\oplus_{j \geq i-1} M_{j}$ for any $i>0$, we have

$$
N^{n+1} \in \text { add } \oplus_{j \geq n} \text { add } M_{j}=0 .
$$

Hence id $X \leq n$. Since this works for any $X$ we have shown gld $\operatorname{End}(\widehat{M})^{\text {op }} \leq$ $n$.

Here we are mainly interested in the representation dimension, therefore we only consider the case $M=\Lambda \oplus \Lambda^{*}$.

6.2 Corollary (shown similarly by Iyama [9]). Let $\Lambda$ be a finite dimensional algebra with quiver

$$
\stackrel{\circ}{\circ} \stackrel{\left(a_{1}, b_{1}\right)}{\longrightarrow} \underset{2}{\circ} \stackrel{\left(a_{2}, b_{2}\right)}{\longrightarrow} \cdots \stackrel{\left(a_{L-1}, b_{L-1}\right)}{\longrightarrow} \stackrel{\circ}{\circ}
$$

for arbitrary $a_{i}, b_{i} \in \mathbb{N}$. That means that $\Lambda$ has exactly $L$ non-isomorphic simple modules $S_{1}, \ldots S_{L}$ and $\operatorname{Ext}_{\Lambda}^{1}\left(S_{v}, S_{w}\right)=0$ whenever $v \neq w-1$. Denote by $I_{l}$ the injective module corresponding to vertex $l$. Assume

(1) $\operatorname{End}_{\Lambda} J^{i} \Lambda$ is semisimple for any $i$.

(2) there is $1 \leq L_{0} \leq L$ such that

(a) $I_{l}$ is projective for all $l>L_{0}$, and

(b) all composition factors of Soc $\Lambda$ are among the simple modules corresponding to vertices $L_{0}, \ldots, L$.

Then

$\operatorname{repdim} \Lambda \leq \max \left\{\operatorname{LL} \Lambda, \max \left\{\operatorname{LL} I_{l}+1 \mid I_{l}\right.\right.$ not projective $\}$

Proof. Set $V=\left\{v \in\{1, \ldots, L\} \mid I_{v}\right.$ not projective $\}$. We may assume that $L_{0} \in V$. We apply Iyama's Theorem with $M_{0}=\Lambda \oplus \bigoplus_{v \in V} I_{v}$. We will show that $M_{i}=J^{i} \Lambda \oplus \bigoplus_{v \in V} I_{v}^{i}$, for submodules $I_{v}^{i} \subseteq I_{v}$ with LL $I_{v}^{i} \leq$ $\max _{v \in V}$ LL $I_{v}+1-i$.

Clearly the construction in Iyama's Theorem respects the direct sum decomposition of $M_{0}$.

We first look at morphisms to the submodules of the indecomposable injective non-projective modules. 
Let $v \in V$ and $\varphi \in \operatorname{Hom}\left(J^{i} \Lambda, I_{v}^{i}\right)$. Since $I_{v}$ is injective $\varphi$ extends to a map $\Lambda \longrightarrow I_{v}$ as indicated in the following diagram.

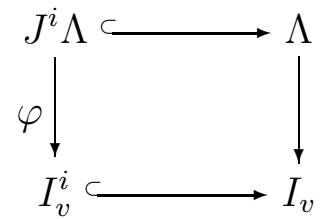

Therefore the image of $\varphi$ is contained in $J^{i} I_{v}$. For the converse let $\psi$ : $\Lambda^{n} \longrightarrow I_{v}$ be a projective cover. Since $I_{v}$ is not projective this is in the radical of $\Lambda$-mod, so $I_{v}^{1}=I_{v}$. Since embedding the radical is in the radical of $\Lambda$-mod so is the composition with the restriction of $\psi$ to some radical power

$$
J^{i} \Lambda^{n} \stackrel{\psi}{\longrightarrow} J^{i} I_{v} \longrightarrow J^{i-1} I_{v}
$$

Therefore one can see, by induction over $i$, that $J^{i} \Lambda \cdot \operatorname{Rad}_{\Lambda \text {-mod }}\left(J^{i} \Lambda, I_{v}^{i}\right)=J^{i} I_{v}$ and $J^{i-1} I_{v} \subseteq I_{v}^{i}$. Especially $I_{L_{0}}^{i}=J^{i-1} I_{L_{0}}$.

Now let $v, w \in V$ with $v \neq w$. Any map $\varphi: I_{v}^{i} \longrightarrow I_{w}^{i}$ has the socle of $I_{v}^{i}$ in its kernel. Therefore the length of the image is at most the length of $I_{v}^{i}$ minus one. By induction on $i$ one obtains LL $I_{v}^{i} \leq \max _{w \in V} \operatorname{LL} I_{w}+1-i$ as claimed above.

Now we want to consider maps to the projective modules.

The composition of a projective cover with embedding of the radical $\Lambda^{(r)} \longrightarrow J \Lambda \hookrightarrow \Lambda$ restricts to maps $J^{i} \Lambda^{(r)} \longrightarrow J^{i+1} \Lambda \hookrightarrow J^{i} \Lambda$. These are in the radical of End $J^{i} \Lambda$ since the second map is in the radical of $\Lambda$-mod. Therefore, together with Assumption (1), we get $J^{i} \Lambda \cdot \operatorname{Rad}_{\operatorname{End}} J^{i} \Lambda=J^{i+1} \Lambda$.

For $v<L_{0}$ we have $\operatorname{Hom}_{\Lambda}\left(I_{v}^{i}, J^{i} \Lambda\right)=0$, since $I_{v}^{i}$ and Soc $J^{i} \Lambda$ do not have any common composition factors. By looking at the composition factors, we can also see that any non-zero element of $\operatorname{Hom}_{\Lambda}\left(I_{L_{0}}^{i}, J^{i} \Lambda\right)$ is a monomorphism. Now assume such a monomorphism exists. Remember that $I_{L_{0}}^{i}=J^{i-1} I_{L_{0}}$. Therefore the simple module corresponding to vertex $L_{0}-\left(\operatorname{LL} I_{L_{0}}-1\right)+$ $(i-1)=L_{0}-\operatorname{LL} I_{L_{0}}+i$ is a composition factor of $I_{L_{0}}^{i}$. So it also is a composition factor of $J^{i} \Lambda$. Let $w$ be a vertex such that it is a composition factor of $J^{i} P_{w}$, where $P_{w}$ is the projective module corresponding to vertex $w$. Then $L_{0}-\operatorname{LL} I_{L_{0}}+i \geq w+i$, so $L_{0}-w \geq \operatorname{LL} I_{L_{0}}$. But if the simple module corresponding to $L_{0}$ is a composition factor of $P_{w}$ then the simple module corresponding to $w$ is a composition factor of $I_{L_{0}}$. Therefore LL $I_{L_{0}} \geq$ $L_{0}-w+1$. A contradiction. Therefore $\operatorname{Hom}_{\Lambda}\left(I_{L_{0}}^{i}, J^{i} \Lambda\right)=0$.

Putting everything together we find that $M_{i}$ has the structure claimed above. Especially $M_{\max \left\{\operatorname{LL} \Lambda, \max \left\{\operatorname{LL} I_{v}+1 \mid I_{v} \text { not projective }\right\}\right.}=0$, so Iyama's Theorem provides the claim of the corollary. 
6.3 Corollary. Let $\Lambda$ be a local finite dimensional algebra. Assume

(1) $J^{i} \Lambda \cdot \operatorname{Rad} \operatorname{End}_{\Lambda} J^{i} \Lambda \subseteq J^{i+1} \Lambda$ for any $i$,

(2) the socle and radical series coincide for $\Lambda$ and for $\Lambda^{*}$, and

(3) any map $\operatorname{Soc}^{3} \Lambda^{*} \longrightarrow \Lambda$ has semisimple image.

Then $\operatorname{repdim} \Lambda \leq \operatorname{LL} \Lambda+1$.

Proof. We may assume that $\Lambda$ is not self-injective (otherwise it is semisimple by Assumption (3)). We set $M_{0}=\Lambda \oplus \Lambda^{*}$ and claim that $M_{i}=J^{i} \Lambda \oplus J^{i-1} \Lambda^{*}$.

As in the proof of Corollary 6.2 we can see that

$$
J^{i} \Lambda^{*} \subseteq J^{i} \Lambda \oplus J^{i-1} \Lambda^{*} \cdot \operatorname{Rad}_{\Lambda-\bmod }\left(J^{i} \Lambda \oplus J^{i-1} \Lambda^{*}, J^{i-1} \Lambda^{*} \subseteq \operatorname{Soc}^{\mathrm{LL} \Lambda-i} \Lambda^{*} .\right.
$$

Since both sides coincide by Assumption (2) we have equality.

The proof of $J^{i} \Lambda \cdot \operatorname{Rad} \operatorname{End}_{\Lambda} J^{i} \Lambda=J^{i+1} \Lambda$ is also identical to the proof of this equality in the case of Corollary 6.2.

It remains to show that $J^{i-1} \Lambda^{*} \cdot \operatorname{Hom}_{\Lambda}\left(J^{i-1} \Lambda^{*}, J^{i} \Lambda\right) \subseteq J^{i+1} \Lambda$. Unfortunately this will clearly fail for $i=\operatorname{LL} \Lambda-1$. But in that case the image of any morphism to $j^{i} \Lambda$ has semisimple image (since the module is semisimple), and the simple module is a direct summand of $M_{i+1}$ anyway, so it still agrees with our claim above. Now assume $i<\operatorname{LL} \Lambda-1$. Let $\varphi \in \operatorname{Hom}_{\Lambda}\left(J^{i-1} \Lambda^{*}, J^{i} \Lambda\right)$. Now we consider the following composition

$$
\operatorname{Soc}^{3} \hookrightarrow J^{i-1} \Lambda^{*} \stackrel{\varphi}{\longrightarrow} J^{i} \Lambda \subset \Lambda \text {. }
$$

By Assumption (3) this composition has semisimple image, so $\varphi$ factors through $J^{i-1} \Lambda^{*} \longrightarrow J^{i-1} \Lambda^{*} / \operatorname{Soc}^{2} \Lambda^{*}$. Therefore the image has Loewy length $\operatorname{LL} \Lambda-(i-1)-2=\operatorname{LL} \Lambda-i-1$, so it is contained in $\operatorname{Soc}^{\operatorname{LL} \Lambda-i-1} \Lambda=J^{i+1} \Lambda$.

6.4 Remark / Corollary. Auslander's result that the representation dimension of a self-injective algebra is bounded above by its Loewy length (see Lemma 2.1.8 or [3]) also follows from Iyama's Theorem (similar to and easier than Corollary 6.3). 


\section{New and improved examples of algebras of representation dimension at least four}

This section is devoted to demonstrating how the results of Sections 5 and 6 can be applied to some interesting classes of algebras. We reprove and generalize Rouquier's result on the representation dimension of exterior algebras, and find a general lower bound for the representation dimension of commutative algebras. By results of 5.3, we automatically also get lower bounds for coverings and certain variations of the algebras presented in this section. Applying the results of Section 6 we find upper bounds for the representation dimension of these algebras. In most cases it will turn out that we actually have identified the representation dimension or that there is only a small number of possible values left.

Most examples will consist of families of algebras indexed by $L$ and $N$, such that $L$ is the maximal Loewy length and $N$ is the number of generators (this will make sense in the actual examples).

\subsection{Examples related to exterior algebras}

As a first example, we consider the exterior algebras, which have been treated by Rouquier [16]. We allow more generally to cut off certain powers of the radical.

7.1.1 Theorem. Let $\Lambda_{L, N}$ be the exterior algebra of an $N$-dimensional vector space modulo the $L$-th power of the radical $(L>1$, note that if $L>N$ then the actual value of $L$ does not matter and the Loewy length is $N+1)$. That is

$\Lambda_{L, N}=k\left\langle x_{1}, \ldots, x_{N}\right\rangle /\left(x_{m} x_{n}+x_{n} x_{m}, x_{n}^{2}, x_{n_{1}} \cdots x_{n_{L}} \mid 1 \leq m, n, n_{i} \leq N\right)$.

- If $L<N$ then

$$
\operatorname{dim} \Lambda_{L, N}-\bmod =\operatorname{dim} D^{b}\left(\Lambda_{L, N}-\bmod \right)=L-1
$$

and

$$
\operatorname{repdim} \Lambda_{L, N}=L+1
$$

- For $L=N$

$$
\operatorname{dim} \Lambda_{N, N}-\bmod =\operatorname{dim} D^{b}\left(\Lambda_{N, N}-\bmod \right)=N-1
$$

and

$$
\operatorname{repdim} \Lambda_{N, N} \geq N+1 \text {. }
$$


- If $L>N$ then

$$
\operatorname{dim} \Lambda_{L, N}-\bmod =N-1, \quad \operatorname{dim} D^{b}\left(\Lambda_{L, N}-\bmod \right)=N
$$

and

$$
\operatorname{repdim} \Lambda_{L, N}=N+1 .
$$

We break the proof up into some lemmas.

7.1.2 Lemma. Let $\Lambda_{L, N}$ as above. Then

$$
\operatorname{dim} \Lambda_{L, N}-\bmod \geq \min \{L-1, N-1\} .
$$

Proof. We want to apply Theorem 3. Set $d=\min \{L-1, N-1\}$. Take $I^{0}=\cdots=I^{H}=\Lambda_{L, N}^{*}$ and $\partial_{i}^{j}$ the map induced by right multiplication by $x_{i+1}$. By definition they fulfill assumptions (1) and (2) of Theorem 3. So consider the diagram

$$
\begin{array}{r}
\Lambda_{L, N}^{*} \otimes_{k} \widehat{k} \stackrel{x_{1}+\sum_{i=1}^{d} x_{i+1} \alpha_{i}}{\longrightarrow} \Lambda_{L, N}^{*} \otimes_{k} \widehat{k} \\
\left.\right|_{x_{2} \cdots x_{d+1}} \\
\Lambda_{L, N}^{*} \otimes_{k} \widehat{k} \stackrel{x_{1}+\sum_{i=1}^{d} x_{i+1} \alpha_{i}}{\longrightarrow} \Lambda_{L, N}^{*} \otimes_{k} \widehat{k}
\end{array}
$$

The vertical map of complexes is not null-homotopic. Therefore

$$
\operatorname{dim} \Lambda_{L, N}-\bmod \geq d
$$

as claimed.

7.1.3 Lemma. Let $\Lambda_{N+1, N}$ be the exterior algebra as in the special case $L=N+1$ above. Then

$$
\operatorname{dim} D^{b}\left(\Lambda_{N+1, N}-\bmod \right)=N .
$$

Proof. For simplicity we write $\Lambda_{N}=\Lambda_{N+1, N}$.

By Lemma 2.2.4 we know $\operatorname{dim} D^{b}\left(\Lambda_{N}\right.$-mod $) \leq N$.

For the other inequality assume $M \in D^{b}\left(\Lambda_{N}\right.$-mod). As in the proof of Lemma 7.1.2 one can see that for any $w \in \mathbb{N}$ there is $x \in \oplus k x_{i}$ such that the (vertical) map

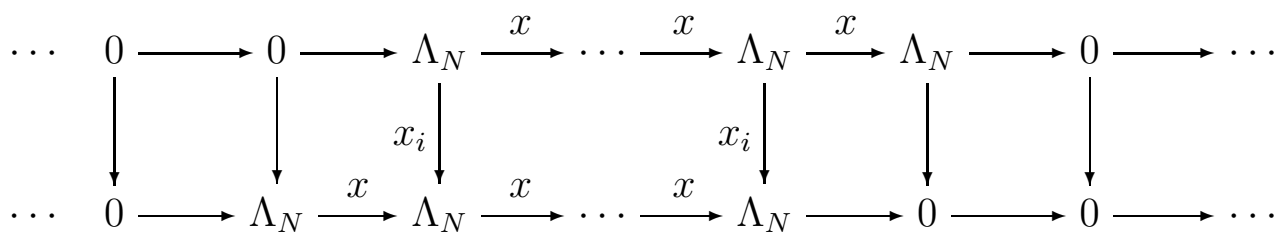

degree: $\quad 0 \quad w$ 
is annihilated by all maps from shifts of $M$. Assume that the homology of $M$ is concentrated in degrees $a, \ldots, b$. Choose $w>b-a+N$. We want to show that the map

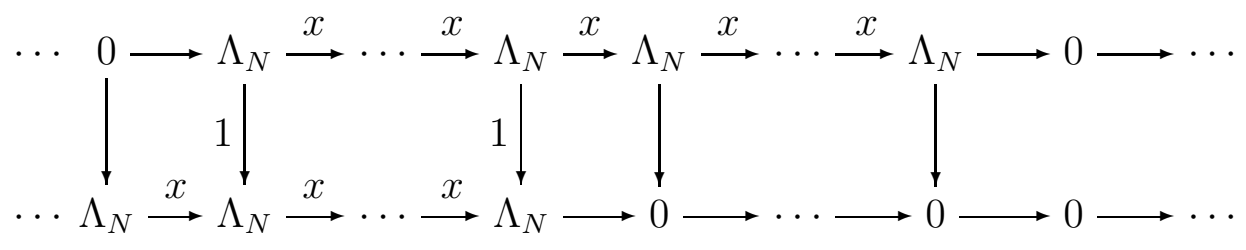

$$
\text { degree: } \quad-N \quad 0 \quad w-N
$$

also annihilates all morphisms from shifts of $M$. Let $i \in \mathbb{N}$. We will show that the map annihilates all morphisms from $M[i]$ by looking at the following two cases:

Case $i<a$ : $M$ may be assumed to be a complex concentrated in degrees $a, \ldots, b$. Therefore $M[i]$ is concentrated in degrees $a-i, \ldots b-i$. Since the upper object is a finite complex of injectives, all morphisms from $M[i]$ to it are represented by morphisms of complexes. Then they annihilate the map above.

Case $i \geq a$ : $M$ may be assumed to be a complex of projectives concentrated in degrees $\leq b$. Therefore $M[i]$ is a complex of projectives concentrated in degrees $\leq b-i \leq b-a<w-N$. Now we can see the map above as the composition $\left[\Lambda_{N} \longrightarrow \cdots \longrightarrow \Lambda_{N}\right] \longrightarrow \Lambda_{N} / \Lambda_{N} x[-(w-N)] \longrightarrow \Lambda_{N} / \Lambda_{N} x$. But $M[i]$ does not have any non-zero morphisms to $\Lambda_{N} / \Lambda_{N} x[-(w-N)]$. Therefore all morphisms from $M[i]$ annihilate the composition.

Now compose $N-1$ maps of the upper type (with different $x_{i}$ - shifted to the correct position) and the lower map and apply Corollary 2.2.10.

7.1.4 Lemma. Let $\Lambda_{N+1, N}$ be as above. Then

$$
\operatorname{repdim} \Lambda_{N+1, N} \leq N+1 \text {. }
$$

Proof. This is just Lemma 2.1.8.

7.1.5 Proposition. Let $\Lambda_{L, N}$ be as above with $L<N$. Then

$$
\operatorname{repdim} \Lambda_{L, N} \leq L+1 \text {. }
$$

Let us start by checking Assumption (1) of Corollary 6.3 for the exterior algebra.

7.1.6 Lemma. Let $\Lambda_{N}=\Lambda_{N+1, N}$ be the exterior algebra. Let $0 \leq i \leq j-2 \leq$ $N-1$. Then

$$
\operatorname{End}_{\Lambda_{N}} J^{i} \Lambda_{N} / J^{j} \Lambda_{N}=k \oplus \operatorname{Hom}_{\Lambda_{N}}\left(J^{i} \Lambda_{N} / J^{j} \Lambda_{N}, J^{i+1} \Lambda_{N} / J^{j} \Lambda_{N}\right) \iota
$$

where $\iota$ is the natural embedding. 
Proof. Since we are looking at a graded module the endomorphism ring is also graded. Therefore we only have to verify that all degree 0 endomorphisms are multiplication by a scalar.

If $i=0$ or $j=N+1$ this is true, since the endomorphisms induce endomorphisms of the simple head $(i=0)$ or simple socle $(j=N+1)$. Therefore we may exclude these cases in the next step.

Assume $1 \leq i$ and $j \leq N$. Let $\varphi: J^{i} \Lambda_{N} / J^{j} \Lambda_{N} \longrightarrow J^{i} \Lambda_{N} / J^{j} \Lambda_{N}$ be a degree 0 morphism. We want to show now that $\varphi$ maps $x_{n} J^{i-1} \Lambda_{N} / J^{j} \Lambda_{N}$ to itself for any $n$. Let $p \in J^{i-1} \Lambda_{N}$ be an element of degree $i-1$. Then

$$
\begin{aligned}
x_{m} \varphi\left(x_{n} p+J^{j} \Lambda_{N}\right) & =\varphi\left(x_{m} x_{n} p+J^{j} \Lambda_{N}\right)=-x_{n} \varphi\left(x_{m} p+J^{j} \Lambda_{N}\right) \\
& \in x_{n} \cdot J^{i} \Lambda_{N} / J^{j} \Lambda_{N} .
\end{aligned}
$$

Let

$$
\varphi\left(x_{n} p+J^{j} \Lambda_{N}\right)=\sum_{n_{1}<n_{2}<\cdots<n_{i}} \alpha_{n_{1}, \ldots, n_{i}} x_{n_{1}} \cdots x_{n_{i}} .
$$

Then

$$
x_{m} \varphi\left(x_{n} p+J^{j} \Lambda_{N}\right)=\sum_{\substack{n_{1}<n_{2}<\cdots<n_{i} \\ n_{1} \neq m, \cdots, n_{i} \neq m}} \alpha_{n_{1}, \ldots, n_{i}} x_{m} x_{n_{1}} \cdots x_{n_{i}}
$$

Therefore each monomial with a nonzero coefficient in $\varphi\left(x_{n} p+J^{j} \Lambda_{N}\right)$ contains at least one of $x_{n}$ and $x_{m}$. Since this works for any $m \neq n$ each such monomial contains $x_{n}$ or all other $x_{m}$. The latter case cannot occur, since $i<N-1$, so $\varphi$ maps $x_{n} J^{i-1} \Lambda_{N} / J^{j} \Lambda_{N}$ to itself as claimed above.

Now we show by induction on $i$ and simultaneously for all $N>i$ that any degree 0 morphism $\varphi: J^{i} \Lambda_{N} / J^{j} \Lambda_{N} \longrightarrow J^{i} \Lambda_{N} / J^{j} \Lambda_{N}$ is multiplication by a scalar.

For $i=0$ this is clear, so assume $i>0$. Then we know that $\varphi$ maps $x_{n} \cdot J^{i-1} \Lambda_{N} / J^{j} \Lambda_{N}$ to itself. Now $x_{n} \cdot J^{i-1} \Lambda_{N} / J^{j} \Lambda_{N}=J^{i-1} \Lambda_{N-1} / J^{j-1} \Lambda_{N-1}$, where the $\Lambda_{N-1}$ is to be interpreted as the exterior algebra on the vector space generated by $x_{m}$ with $m \neq n$. Now inductively $\left.\varphi\right|_{x_{n} \cdot J^{i-1} \Lambda_{N} / J^{j} \Lambda_{N}}$ is multiplication by some $\alpha_{n}$, and the $\alpha_{n}$ all coincide since the $x_{n} \cdot J^{i-1} \Lambda_{N} / J^{j} \Lambda_{N}$ have pairwise non-trivial intersection.

Therefore our morphism is multiplication by a scalar, and the claim of the lemma follows.

Proof of Proposition 7.1.5. We would like to apply Corollary 6.3. We have verified Claim (1) in Lemma 7.1.6 above, and Claim (2) is obvious. To verify Claim (3) let $\varphi: \operatorname{Soc}^{3} \Lambda_{L, N}^{*} \longrightarrow \Lambda_{L, N}$. The monomials of the form $x_{n_{1}} \cdots x_{n_{i}}$ 
with $n_{1}<\cdots<n_{i}$ and $i<L$ form a basis of $\Lambda_{L, N}$. We consider the dual basis of $\Lambda_{L, N}^{*}$. Then

$$
\operatorname{Soc}^{3} \Lambda_{L, N}^{*}=\bigoplus_{\substack{m \text { such a } \\ \text { monomial of } \\ \text { degree } \leq 2}} k m^{*}
$$

Now note that for $n<m$ we have $x_{r}\left(x_{n} x_{m}\right)^{*}=0$ for all $r \notin\{n, m\}$. Therefore all these $x_{r}$ have to operate as zero on $\varphi\left(\left(x_{n} x_{m}\right)^{*}\right)$. It follows that $\varphi\left(\left(x_{n} x_{m}\right)^{*}\right) \in J^{N-2} \Lambda_{L, N}+\operatorname{Soc} \Lambda_{L, N}$. Since Soc $\Lambda_{L, N}=J^{L-1} \Lambda_{L, N}$, Claim (3) follows for $N-2 \geq L-1$, that is $L \leq N-1$.

Proof of Theorem 7.1.1. The claims of Theorem 7.1.1 follow from the inequalities in Lemmas 7.1.2, 7.1.3, 7.1.4, Proposition 7.1.5 and in 4.6

7.1.7 Remark. Unfortunately, we are not able to identify the representation dimension for $L=N$. It seem reasonable to expect that repdim $\Lambda_{N, N}=$ repdim $\Lambda_{N+1, N}=N+1$, since the representation theory of these two algebras almost coincides.

Note that the algebras $\Lambda_{L, N}$ of Theorem 7.1.1 are $\mathbb{Z}$-graded by deg $x_{i}=1$. Therefore automatically also get lower bounds for the following covering.

7.1.8 Theorem. Let $\Lambda_{L, N}$ be the exterior algebra of an $N$-dimensional vector space modulo the $L$-th power of the radical, as treated in Theorem 7.1.1. In the notation of 5.3 we set $\widetilde{\Lambda}_{L, N}=\left(\Lambda_{L, N}\right)_{\{1, \ldots, L\}}$, that is the covering with respect to the subset $\{1, \ldots, L\} \subset \mathbb{Z}$. Then $\widetilde{\Lambda}_{L, N}=k Q / I$ with

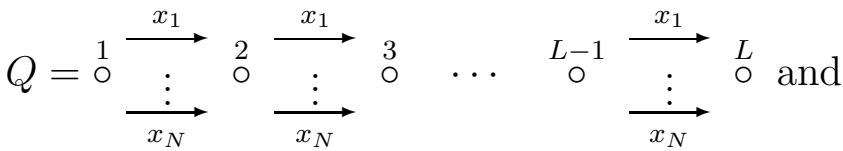

$$
\begin{aligned}
& I=\left(x_{n} x_{m}+x_{m} x_{n}, x_{n}^{2}\right) \text {. }
\end{aligned}
$$

Then

$$
\operatorname{dim} \widetilde{\Lambda}_{L, N}-\bmod =\min \{L-1, N-1\}
$$

and

$$
\operatorname{repdim} \widetilde{\Lambda}_{L, N}=\min \{L+1, N+1\} .
$$

Moreover, for $L \leq N$

$$
\operatorname{dim} D^{b}\left(\widetilde{\Lambda}_{L, N}-\bmod \right)=L-1
$$

and otherwise

$$
\operatorname{dim} D^{b}\left(\widetilde{\Lambda}_{L, N}-\bmod \right) \in\{N-1, N\}
$$


7.1.9 Lemma. Let $\widetilde{\Lambda}_{L, N}$ as above. Then $\operatorname{repdim} \widetilde{\Lambda}_{L, N} \leq \min \{L+1, N+1\}$.

Proof. We want to apply Corollary 6.2. One can see that Assumption (1) is satisfied as in the proof of Lemma 7.1.6, except that we do not have to restrict to degree 0 morphisms (since there are no morphisms of non-zero degree). If we choose $L_{0}=\min \{L, N\}$ then Assumption (2) holds.

Proof of Theorem 7.1.8. By Proposition 5.3.1 and the proof of Lemma 7.1.2 we have $\operatorname{dim} \widetilde{\Lambda}_{L, N}-\bmod \geq \min \{L-1, N-1\}$. Now the theorem follows with the inequality if Lemma 7.1 .9 above and the inequalities of 4.6.

Finally we apply Corollary 5.3.2. Note that a group homomorphism from $\mathbb{Z}$ is given by the image of 1 . Further note that any graded automorphism of $\Lambda_{L, N}$ comes from an automorphism of the vector space $k x_{1} \oplus \cdots \oplus k x_{N}$, and therefore is given by an invertible $N \times N$-matrix.

7.1.10 Theorem. Let $A=\left(a_{i j}\right)$ be an invertible $N \times N$-matrix over $k$. Let $\Lambda_{L, N}^{A}$ be the algebra

$$
\begin{array}{cr}
\Lambda_{L, N}^{A}=k\left\langle x_{1}, \ldots, x_{N}\right\rangle /\left(\sum_{i} a_{m i} x_{n} x_{i}+\sum_{i} a_{n i} x_{m} x_{i}\right. & 1 \leq m, n \leq N, \\
\sum_{i} a_{n i} x_{n} x_{i} & 1 \leq n \leq N, \\
x_{n_{1}} \cdots x_{n_{L}} & \left.1 \leq n_{i} \leq N\right) .
\end{array}
$$

Then

$\min \{L-1, N-1\} \leq \operatorname{dim} \Lambda_{L, N}^{A}-\bmod \leq \operatorname{dim} D^{b}\left(\Lambda_{L, N}^{A}-\bmod \right) \leq \min \{L-1, N\}$, and in particular

$$
\operatorname{repdim} \Lambda_{L, N}^{A} \geq \min \{L+1, N+1\}
$$

7.1.11 Example. In Theorem 7.1 .10 above, let $N=3, L=4$ and $A=$ $\left(\begin{array}{ccc}s t & & \\ & t & \\ & & \end{array}\right)$ with $s, t \in k \backslash\{0\}$. Then we find

$$
\operatorname{repdim}\left(k\langle x, y, z\rangle /\left(x^{2}, y^{2}, z^{2}, x y+s y x, x z+s t z x, y z+t z y\right)\right) \geq 4 \text {. }
$$

\subsection{Examples related to truncated polynomial rings}

7.2.1 Theorem. Let $\Sigma_{L, N}=k\left[x_{1}, \ldots x_{N}\right] /\left(x_{1}, \ldots, x_{N}\right)^{L}$. That is the polynomial ring in $N$ variables modulo all polynomials of degree $L$. 
- Assume $L \leq N$. Then

$$
\operatorname{dim} \Sigma_{L, N}-\bmod =\operatorname{dim} D^{b}\left(\Sigma_{L, N}-\bmod \right)=L-1,
$$

and

$$
\operatorname{repdim} \Sigma_{L, N}=L+1 \text {. }
$$

- Assume $L>N$. Then

$$
N-1 \leq \operatorname{dim} \Sigma_{L, N}-\bmod \leq \operatorname{dim} D^{b}\left(\Sigma_{L, N}-\bmod \right) \leq L-1,
$$

and

$$
N+1 \leq \operatorname{repdim} \Sigma_{L, N} \leq L+1
$$

7.2.2 Remark. The case $N=1$ suggests that in the the correct number for the representation dimension could always be $\min \{L+1, N+1\}$.

As before, we break the proof into several smaller lemmas.

7.2.3 Lemma. Let $\Sigma_{L, N}$ be as above. Then

$$
\operatorname{dim} \Sigma_{L, N}-\bmod \geq \min \{L-1, N-1\}
$$

Proof. Set $d=\min \{L-1, N-1\}$. Take $I^{j}=\left(\Sigma_{L, N}^{*}\right)\left(\begin{array}{l}d \\ j\end{array}\right)$, that is $\left(\begin{array}{l}d \\ j\end{array}\right)$ copies of the indecomposable injective module. We assume these copies to be indexed by the subsets of $\{1 \ldots d\}$ having exactly $j$ elements, and write $\left(\sum_{L, N}^{*}\right)^{S}$ with $S \subseteq\{1 \ldots d\}$ and $|S|=j$ for the corresponding direct summand of $I^{j}$. We define the maps $\partial_{i}^{j}$ by giving their components between the direct summands. For $\partial_{0}^{j}$ the component $\left(\Sigma_{L, N}^{*}\right)^{S} \longrightarrow\left(\Sigma_{L, N}^{*}\right)^{T}$ is

$$
\begin{cases}0 & \text { if } S \not \subset T \\ (-1)^{|\{s \in S \mid s<t\}|} x_{t} & \text { if } S \cup\{t\}=T .\end{cases}
$$

For $i>0$ the component $\left(\Sigma_{L, N}^{*}\right)^{S} \longrightarrow\left(\Sigma_{L, N}^{*}\right)^{T}$ of $\partial_{i}^{j}$ is

$$
\begin{cases}0 & \text { if } S \not \subset T \\ (-1)^{|\{s \in S \mid s<t\}|} x_{N} & \text { if } S \cup\{t\}=T .\end{cases}
$$

It is a straightforward calculation to verify that these maps fulfill assumptions (1) and (2) of Theorem 3. By induction on $d^{\prime}$ with $0 \leq d^{\prime} \leq d$ one can see that the map $\partial_{1}^{1} \cdots \partial_{d^{\prime}}^{d^{\prime}}$ is given by its components

$$
\left.\begin{array}{ll}
0 & \text { if } S \neq\left\{1 \ldots d^{\prime}\right\} \\
\pm x_{N}^{d^{\prime}} & \text { if } S=\left\{1 \ldots d^{\prime}\right\}
\end{array}\right\}:\left(\Sigma_{L, N}^{*}\right)^{\emptyset} \longrightarrow\left(\Sigma_{L, N}^{*}\right)^{S}
$$


Therefore we consider, for $\alpha \in \bar{k}^{d}$ and $\widehat{k}=k(\alpha)$, the following vertical map of complexes.

$$
\left.\left(\Sigma_{L, N}^{*}\right)^{d} \otimes_{k} \widehat{k} \stackrel{\left(\begin{array}{c}
x_{1}+\alpha_{1} x_{N} \\
-\left(x_{2}+\alpha_{2} x_{N}\right) \\
\vdots \\
\pm\left(x_{d}+\alpha_{d} x_{N}\right)
\end{array}\right)}{\longrightarrow}\right|_{L, N} ^{*} \otimes_{k} \widehat{k} \stackrel{\left(x_{1}+\alpha_{1} x_{N}, \ldots, x_{d}+\alpha_{d} x_{N}\right)}{\longrightarrow}\left(\Sigma_{L, N}^{*}\right)^{d} \otimes_{k} \widehat{k}
$$

Clearly it is never null-homotopic. Therefore Theorem 3 can be applied and provides the lower bound for $\operatorname{dim} \Sigma_{L, N}$-mod.

7.2.4 Lemma. Let $\Sigma_{L, N}$ be as in Theorem 7.2.1. Then repdim $\Sigma_{L, N} \leq L+1$.

Proof. We want to apply Corollary 6.3. We can see that Assumption (1) holds in a similar way to the proof of Lemma 7.1.6. The differences are that we cannot and don't have to exclude the case $j=L-1$, and that $x_{n} \cdot J^{i-1} \Sigma_{L, N} / J^{j} \Sigma_{L, N}=J^{i-1} \Sigma_{L, N} / J^{j-1} \Sigma_{L, N}$, so we do not need to look at different $N$ simultaneously. Assumption (2) is obvious and Assumption (3) can be seen as in Proposition 7.1.5.

Proof of Theorem 7.2.1. The claims of the theorem now follow from the inequalities in Lemmas 7.2.3 and 7.2.4 and in the diagram in 4.6.

For an ideal $I \unlhd k\left[x_{1}, \ldots, x_{N}\right]$ and $a \in k^{N}$ we say that $I$ has an $L$-fold zero in $a$ if $I \subseteq\left(x_{i}-a_{i} \mid 1 \leq i \leq N\right)^{L}$. Note that for showing the lower bounds for the three dimensions in Theorem 7.2.1 it was only necessary to factor out an ideal which has an $L$-fold zero in 0 . Also we can move the zero to any other point by changing the coordinates. Therefore we have shown

Theorem 4. Let $I \unlhd k\left[x_{1}, \ldots, x_{N}\right]$. Assume that $I$ has an $L$-fold zero. Then

$$
\begin{aligned}
\min \{L-1, N-1\} & \leq \operatorname{dim} k\left[x_{1}, \ldots, x_{N}\right] / I-\bmod \\
& \leq \operatorname{dim} D^{b}\left(k\left[x_{1}, \ldots, x_{N}\right] / I-\bmod \right)
\end{aligned}
$$

and in particular

$$
\operatorname{repdim} k\left[x_{1}, \ldots, x_{N}\right] / I \geq \min \{L+1, N+1\} .
$$

7.2.5 Remark. Avramov and Iyengar [6], using techniques from [5], have announced that the dimension of the stable derived category of a complete intersection local ring $R$ is at least the codimension of $R$ minus one. As a 
corollary they deduce that when in addition the ring is artinian, its representation dimension is at least the embedding dimension plus one. In particular,

$$
\forall c_{1}, \ldots c_{N}>1: \operatorname{repdim} k\left[x_{1}, \ldots, x_{N}\right] /\left(x_{1}^{c_{1}}, \ldots, x_{N}^{c_{N}}\right) \geq N+1
$$

It is worth noting that the result of Theorem 4 intersects their result, where the intersection consists of the cases with $c_{1}, \ldots, c_{N} \geq N$.

Next we look at the following covering of the truncated polynomial rings.

7.2.6 Theorem. Let $\Sigma_{L, N}$ be the as in Theorem 7.2.1. In the notation of 5.3 we set $\widetilde{\Sigma}_{L, N}=\left(\Sigma_{L, N}\right)_{\{1, \ldots, L\}}$, that is the covering with respect to the subset $\{1, \ldots, L\} \subset \mathbb{Z}$. Then $\widetilde{\Sigma}_{L, N}=k Q / I$ with

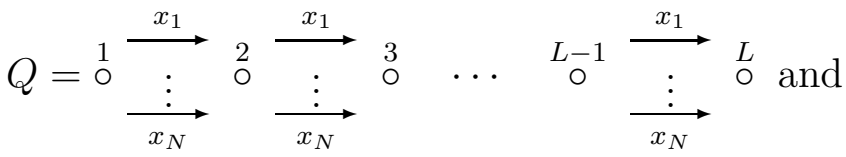

$$
\begin{aligned}
& I=\left(x_{n} x_{m}-x_{m} x_{n}\right) \text {. }
\end{aligned}
$$

- Assume $L \leq N$. Then

$$
\operatorname{dim} \widetilde{\Sigma}_{L, N}-\bmod =\operatorname{dim} D^{b}\left(\widetilde{\Sigma}_{L, N}-\bmod \right)=L-1,
$$

and

$$
\operatorname{repdim} \widetilde{\Sigma}_{L, N}=L+1
$$

- Assume $L>N$. Then

$$
N-1 \leq \operatorname{dim} \widetilde{\Sigma}_{L, N}-\bmod \leq \operatorname{dim} D^{b}\left(\widetilde{\Sigma}_{L, N}-\bmod \right) \leq N,
$$

and

$$
N+1 \leq \operatorname{repdim} \widetilde{\Sigma}_{L, N} \leq L+1
$$

7.2.7 Remark. In case $L=N$ these are the algebras treated by Krause and Kussin. It should be noted that their lower bound for the representation dimension is improved by two here, and that we actually determined all dimensions in that case.

The upper bound for the representation dimension of these algebras has been constructed by Iyama:

7.2.8 Lemma (Iyama). Let $\widetilde{\Sigma}_{L, N}$ be as above. Then

$$
\operatorname{repdim} \widetilde{\Sigma}_{L, N} \leq L+1 \text {. }
$$


Proof. We apply Corollary 6.2. Assumption (1) can again be seen similarly to the proof of Lemma 7.1.6, by combining the changes sketched in the proofs of Lemmas 7.1.9 and 7.2.4. Assumption (2) clearly holds for $L_{0}=L$.

Proof of Theorem 7.2.6. By Proposition 5.3.1 and the proof of Lemma 7.2.3 we have $\operatorname{dim} \widetilde{\Lambda}_{L, N}-\bmod \geq \min \{L-1, N-1\}$. Now the theorem follows with the inequality if Lemma 7.2.8 above and the inequalities of 4.6.

As for the exterior algebras, we also get various algebras which have the same covering here.

7.2.9 Theorem. Let $A=\left(a_{i j}\right)$ be an invertible $N \times N$-matrix over $k$. Let $\Sigma_{L, N}^{A}$ be the algebra

$$
\begin{gathered}
\Sigma_{L, N}^{A}=k\left\langle x_{1}, \ldots, x_{N}\right\rangle /\left(\sum_{i} a_{m i} x_{n} x_{i}-\sum_{i} a_{n i} x_{m} x_{i},\right. \\
\left.x_{n_{1}} \cdots x_{n_{L}}\right) .
\end{gathered}
$$

Then

$$
\min \{L-1, N-1\} \leq \operatorname{dim} \Sigma_{L, N}^{A}-\bmod \leq \operatorname{dim} D^{b}\left(\Sigma_{L, N}^{A}-\bmod \right) \leq L-1,
$$

and in particular

$$
\operatorname{repdim} \Sigma_{L, N}^{A} \geq \min \{L+1, N+1\}
$$

\subsection{Further examples}

Now we present another two examples of relatively small algebras of representation dimension four.

7.3.1 Theorem. Let $k$ be a field, $\Lambda=k Q / I$ with

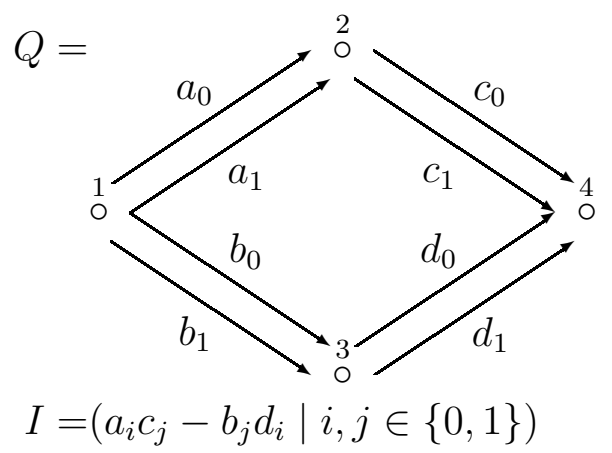

Then

$$
\operatorname{dim} \Lambda-\bmod =\operatorname{dim} D^{b}(\Lambda-\bmod )=2,
$$


and

$$
\operatorname{repdim} \Lambda=4
$$

7.3.2 Lemma. Let $\Lambda$ as above. Then $\operatorname{dim} \Lambda-\bmod \geq 2$.

Proof. We denote by $E_{1}, \ldots E_{4}$ the indecomposable injective modules, and by $\widehat{a_{0}}, \ldots, \widehat{d_{1}}$ the maps between them induced by the arrows of the quiver. Now we apply Theorem 3 with $d=2$ and

$$
\begin{array}{rlrl}
I^{0} & =E_{4} & I^{1} & =E_{2} \oplus E_{3} \\
I^{2} & =E_{1} & \partial_{0}^{1} & =\left(\widehat{c_{0}},-\widehat{d_{0}}\right) \\
\partial_{1}^{1} & =\left(0,-\widehat{d_{1}}\right) & \partial_{2}^{1} & =\left(\widehat{c_{1}}, 0\right) \\
\partial_{0}^{2} & =\left(\begin{array}{c}
\widehat{a_{0}} \\
\widehat{b_{0}}
\end{array}\right) & \partial_{1}^{2}=\left(\begin{array}{c}
\widehat{a_{1}} \\
0
\end{array}\right) \\
\partial_{0}^{2}=\left(\begin{array}{c}
0 \\
\left(\widehat{b_{1}}\right.
\end{array}\right) & &
\end{array}
$$

It is straightforward but somewhat tedious to verify that the assumptions of Theorem 3 are met. In particular, verifying that the map of complexes in Theorem 3 is not null-homotopic here can be done by a brute force approach (assume there is a null-homotopy, write it as linear combination of the maps induced by the arrows, this amounts to a large system of linear equations which can be shown to not be solvable).

7.3.3 Lemma. Let $\Lambda$ as in Theorem 7.3.1. Then

$$
\operatorname{repdim} \Lambda \leq 4
$$

Proof. We apply Iyama's theorem (Theorem 6.1). Here $P_{i}, E_{i}$, and $S_{i}$ denote the indecomposable projective, indecomposable injective and simple module corresponding to vertex $i$ respectively.

$$
\begin{aligned}
& M_{0}=P_{1} \oplus P_{2} \oplus P_{3} \oplus P_{4} \oplus E_{1} \oplus E_{2} \oplus E_{3} \oplus E_{4} \\
& M_{1}=\operatorname{Rad} P_{1} \oplus P_{4} \oplus E_{1} \oplus E_{2} \oplus E_{3} \oplus E_{4} \\
& M_{2}=P_{4} \oplus E_{1} \oplus E_{2} \oplus E_{3} \oplus \operatorname{Rad} E_{4} \\
& M_{3}=P_{4} \oplus E_{1} \oplus S_{2} \oplus S_{3} \\
& M_{4}=0
\end{aligned}
$$

Therefore $\operatorname{repdim} \Lambda \leq 4$.

Proof of Theorem 7.3.1. The claims of the theorem now follow from the inequalities in Lemmas 7.3.2 and 7.3.3 and in the diagram in 4.6. 
7.3.4 Theorem. Let $k$ be a field, $\Lambda=k Q / I$ with

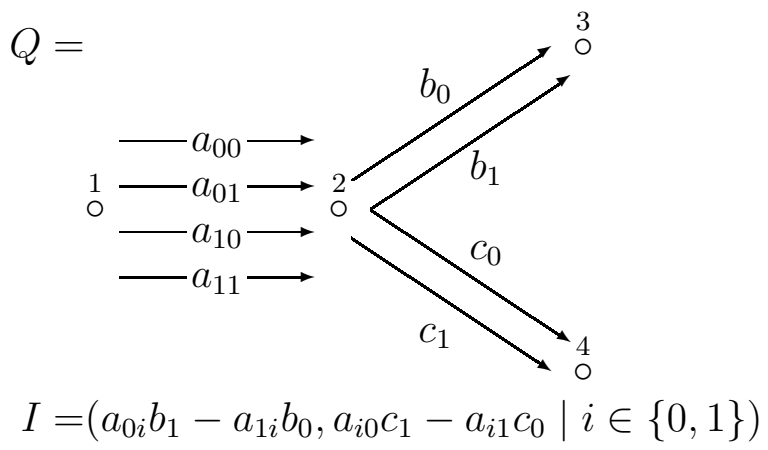

Then

$$
\operatorname{dim} \Lambda-\bmod =\operatorname{dim} D^{b}(\Lambda-\bmod )=2,
$$

and

$$
\operatorname{repdim} \Lambda=4 .
$$

The proof is essentially the same as the one of Theorem 7.3.1. The steps are as follows:

7.3.5 Lemma. Let $\Lambda$ as above. Then $\operatorname{dim} \Lambda-\bmod \geq 2$.

Proof. We denote by $E_{1}, \ldots E_{4}$ the indecomposable injective modules, and by $\widehat{a_{00}}, \ldots, \widehat{c_{1}}$ the maps between them induced by the arrows of the quiver. Now we apply Theorem 3 with $d=2$ and

$$
\begin{aligned}
& I^{0}=E_{3} \oplus E_{4} \quad I^{1}=E_{2}^{3} \\
& I^{2}=E_{1} \\
& \partial_{0}^{1}=\left(\begin{array}{ccc}
\widehat{b_{1}} & 0 & -\widehat{b_{0}} \\
0 & \widehat{c_{1}} & -\widehat{c_{0}}
\end{array}\right) \\
& \partial_{1}^{1}=\left(\begin{array}{ccc}
-\widehat{b_{0}} & 0 & 0 \\
0 & 0 & 0
\end{array}\right) \\
& \partial_{2}^{1}=\left(\begin{array}{ccc}
0 & \widehat{b_{0}} & 0 \\
0 & 0 & 0
\end{array}\right) \\
& \partial_{0}^{2}=\left(\frac{\widehat{a_{01}}}{\frac{\widehat{a 10}}{a_{11}}}\right) \\
& \partial_{1}^{2}=\left(\frac{0}{\frac{a_{00}}{a_{01}}}\right) \\
& \partial_{0}^{2}=\left(\begin{array}{c}
\widehat{a_{00}} \\
0 \\
0
\end{array}\right)
\end{aligned}
$$

As in Lemma 7.3.2 is is straightforward to verify that the assumptions of Theorem 3 are met. However the calculations are even worse.

7.3.6 Lemma. Let $\Lambda$ as in Theorem 7.3.4. Then

$$
\operatorname{repdim} \Lambda \leq 4
$$


Proof. We apply Iyama's theorem (Theorem 6.1). We denote by $P_{i}, E_{i}$, and $S_{i}$ the indecomposable projective, indecomposable injective and simple module corresponding to vertex $i$ respectively.

$$
\begin{aligned}
& M_{0}=P_{1} \oplus P_{2} \oplus P_{3} \oplus P_{4} \oplus E_{1} \oplus E_{2} \oplus E_{3} \oplus E_{4} \\
& M_{1}=\operatorname{Rad} P_{1} \oplus P_{3} \oplus P_{4} \oplus E_{1} \oplus E_{2} \oplus E_{3} \oplus E_{4} \\
& M_{2}=P_{3} \oplus P_{4} \oplus E_{1} \oplus E_{2} \oplus \operatorname{Rad} E_{3} \oplus \operatorname{Rad} E_{4} \\
& M_{3}=P_{3} \oplus P_{4} \oplus E_{1} \oplus S_{2} \\
& M_{4}=0
\end{aligned}
$$

Therefore $\operatorname{repdim} \Lambda \leq 4$.

Proof of Theorem 7.3.4. The claims of the theorem now follow from the inequalities in Lemmas 7.3.5 and 7.3.6 and in the diagram in 4.6. 


\section{Open questions}

This section consists of a list of questions which came up as a consequence of the results presented in this thesis.

1. Is the lower bound we found for the representation dimension of the elementary abelian groups (Theorem 1) the precise value?

2. Are there non-semisimple algebras $\Lambda$, such that equality holds in the inequality repdim $\Lambda \geq \operatorname{dim} D^{b}(\Lambda$-mod) of Proposition 2.2.7?

3. Find examples of algebras with $\operatorname{dim} \Lambda-\bmod +2<\operatorname{repdim} \Lambda$. (We always have " $\leq$ " by Lemmas 4.4 and 4.5.)

4. Can the difference between $\operatorname{dim} D^{b}(\Lambda$-mod $)$ and $\operatorname{dim} \Lambda$-mod get arbitrarily large?

5. Given an algebra $\Lambda$, is there any general way to find a good (or even the best) complex of injective $\Lambda \otimes_{k} R$-lattices for the application of Theorem 2.

6. Is there any upper bound for the representation dimensions of the algebras of a given (finite) global dimension?

7. For $\Lambda_{L, N}$ as in Theorem 7.1.1, is repdim $\Lambda_{N, N}=N+1$ ? More generally, if $\Lambda$ is a basic algebra, $P$ a direct summand of $\Lambda$ which is injective, is $\operatorname{repdim} \Lambda / \operatorname{Soc} P=\operatorname{repdim} \Lambda$ ? (By [8] we have " $\geq$ ".)

8. Is there a connection between representation dimension and tameness? (Note that the criterion in Theorem 2 needs a two parameter family to show that an algebra has representation dimension at least four, so it should not be possible to apply it to show that a tame algebra has representation dimension greater than three.) 


\section{References}

[1] Ibrahim Assem, María Inés Platzeck, and Sonia Trepode, On the representation dimension of tilted and laura algebras, J. Algebra 296 (2006), no. $2,426-439$.

[2] Maurice Auslander, Representation dimension of Artin algebras, Queen Mary College Mathematics Notes (1971), republished in [4].

[3] _ Representation theory of Artin algebras. I, II, Comm. Algebra 1 (1974), 177-268; ibid. 1 (1974), 269-310.

[4] _ Selected works of Maurice Auslander. Part 1, American Mathematical Society, Providence, RI, 1999, Edited and with a foreword by Idun Reiten, Sverre O. Smalø, and Øyvind Solberg.

[5] L. L. Avramov, R.-O. Buchweitz, S. Iyengar, and C. Miller, Homology of perfect complexes, arXiv:math.AC/0609008.

[6] L. L. Avramov and S. Iyengar, The dimension of the stable derived category of a complete intersection local ring, in preparation.

[7] Charles W. Curtis and Irving Reiner, Representation theory of finite groups and associative algebras, Pure and Applied Mathematics, Vol. XI, Interscience Publishers, a division of John Wiley \& Sons, New YorkLondon, 1962.

[8] Karin Erdmann, Thorsten Holm, Osamu Iyama, and Jan Schröer, Radical embeddings and representation dimension, Adv. Math. 185 (2004), no. 1, 159-177.

[9] Osamu Iyama, private communication.

[10] _ Rejective subcategories of artin algebras and orders, preprint, arXiv:math.RT/0311281.

[11] , Finiteness of representation dimension, Proc. Amer. Math. Soc. 131 (2003), no. 4, 1011-1014.

[12] Henning Krause and Dirk Kussin, Rouquier's theorem on representation dimension, Trends in representation theory of algebras and related topics, Contemp. Math., vol. 406, Amer. Math. Soc., Providence, RI, 2006, pp. 95-103. 
[13] Hideyuki Matsumura, Commutative ring theory, Cambridge Studies in Advanced Mathematics, vol. 8, Cambridge University Press, Cambridge, 1989, Translated from the Japanese by M. Reid.

[14] Jeremy Rickard, Derived categories and stable equivalence, J. Pure Appl. Algebra 61 (1989), no. 3, 303-317.

[15] Raphaël Rouquier, Dimensions of triangulated categories, preprint, arXiv:math.CT/0310134.

[16] - Representation dimension of exterior algebras, Invent. Math. 165 (2006), no. 2, 357-367. 


\section{Erklärung}

Ich versichere, dass ich die von mir vorgelegte Dissertation selbständig angefertigt, die benutzten Quellen und Hilfsmittel vollständig angegeben und die Stellen der Arbeit - einschlielich Tabellen, Karten und Abbildungen -, die anderen Werken im Wortlaut oder dem Sinn nach entnommen sind, in jedem Einzelfall als Entlehnung kenntlich gemacht habe; dass diese Dissertation noch keiner anderen Fakultät oder Universität zur Prüfung vorgelegen hat; dass sie - abgesehen von unten angegebenen Teilpublikationen - noch nicht veröffentlicht worden ist sowie, dass ich eine solche Veröffentlichung vor Abschluss des Promotionsverfahrens nicht vornehmen werde. Die Bestimmungen dieser Promotionsordnung sind mir bekannt. Die von mir vorgelegte Dissertation ist von Prof. Dr. Steffen König betreut worden.

\section{Teilpublikation:}

A lower bound for the representation dimension of $k C_{p}^{n}$, Math. Z. 256 (2007), no. 3, 481-490. 